Courrier du Centre international Blaise Pascal

\title{
Les Copies des Pensées
}

\section{Gilles Proust}

\section{OpenEdition}

\section{Journals}

Édition électronique

URL : http://journals.openedition.org/ccibp/325

DOI : $10.4000 /$ ccibp.325

ISSN : 2493-7460

\section{Éditeur}

Centre international Blaise Pascal

\section{Édition imprimée}

Date de publication : 6 décembre 2010

Pagination : 4-47

ISBN : 978-2-84516-498-7

ISSN : 0249-6674

Référence électronique

Gilles Proust, "Les Copies des Pensées ", Courrier du Centre international Blaise Pascal [En ligne], 32 | 2010, mis en ligne le 26 novembre 2015, consulté le 02 mai 2019. URL : http://

journals.openedition.org/ccibp/325 ; DOI : 10.4000/ccibp.325

Ce document a été généré automatiquement le 2 mai 2019.

Centre international Blaise Pascal 


\title{
Les Copies des Pensées
}

\author{
Gilles Proust
}

1 L'objet de cette étude est de présenter un état des recherches récemment poursuivies sur les papiers originaux et les copies des Pensées en vue de l'édition électronique entreprise au sein du C.E.R.H.A.C'

2 La Bibliothèque Nationale de France possède trois manuscrits des Pensées qui proviennent de la famille de Pascal : le Recueil des papiers originaux des Pensées de Pascal ( $m s 9202$ fonds français - anciennement $n^{\circ} 3002$ du supplément français), et deux copies manuscrites établies après la mort de Pascal : une première Copie (notée $C_{1}$ par Jean Mesnard) cotée ms 9203 fonds français anciennement $n^{\circ} 3002$ bis du supplément français et une deuxième Copie (notée $C_{2}$ ) intégrée au Recueil ms 12449 du fonds français anciennement $n^{\circ} 176$ du suppl. français (noté $\mathrm{RC}_{2}$ ) ; cette dernière Copie est suivie d'une copie intitulée La loi était figurative, écrite sur un cahier à part ${ }^{2}$.

\section{Historique sommaire}

3 Pour le lecteur des $\mathrm{XVII}^{\mathrm{e}}$ s. et XVIII ${ }^{\mathrm{e}}$ s., l'ordre et le texte des Pensées étaient déterminés par l'ouvrage publié pour la première fois en janvier $1670^{3}$ et plus précisément par le texte de l'édition de 1678, augmenté de quelque quarante pensées.

4 Que ce soit dans les éditions de Condorcet (1776) dont le texte était parfois mutilé, de Bossut (1779) dont le classement se voulait thématique, ou de Renouard (1812) qui reproduisait en grande partie l'édition Bossut, le texte était toujours tiré de l'édition de 1678. Seules quelques pensées inédites, découvertes dans une copie établie par l'abbé Périer $^{4}$, et publiées partiellement pour la première fois par le père Pierre Nicolas Desmolets $(1728)^{5}$, étaient proposées en supplément dans ces éditions. Pourtant Charles Bossut dit lui-même dans son Discours sur la vie de Pascal que Guerrier de Bezance, neveu de Pierre Guerrier ${ }^{6}$, lui avait prêté des copies inédites (probablement la troisième copie Guerrier ${ }^{7}$ et le Recueil $\left.\mathrm{RC}_{2}{ }^{8}\right)^{9}$. Augustin Renouard montre aussi dans son 
introduction qu'il connaissait l'existence du Recueil des originaux et au moins la Copie $C_{1}$ qu'il attribuait par erreur à Pierre Guerrier ${ }^{10}$.

5 Le Rapport de Victor Cousin à l'Académie française en 1842 (publié en 1843) critique fortement Bossut et l'édition de Port-Royal. Il demande que le texte publié soit établi à partir des manuscrits existants et en priorité par la transcription des papiers originaux. C'est ce que fait Prosper Faugère en 1844. Mais personne n'a encore conscience de l'importance de l'ordre proposé par les Copies. Faugère propose toujours une édition thématique et si Ernst Havet (1851-1866) adopte le texte de Faugère, il suit en grande partie l'ordre de l'édition Bossut. Léon Brunschvicg (1904) propose aussi une édition thématique qui sépare la partie anthropologique de la partie religieuse. Il considère aussi avec Gustave Michaut (1896) que la Copie originale mentionnée par Étienne Périer dans la Préface de l'édition de 1670 a disparu, et que $C_{1}$ (qu'ils appellent la Copie) a été réalisée par le comité de Port-Royal en vue d'une édition. Selon eux les fragments classés étaient ceux que l'on destinait à la publication. Michaut a publié en 1895 un texte revu sur les originaux et classé dans l'ordre du Recueil de ces originaux.

2, Zacharie Tourneur" préfere le classement de la Copie $C_{1}$, "classement qu'il ne s'est pas cru obligé de suivre servilement ", car il semble convaincu que $C_{1}$ est la première copie dont parle Étienne Périer. C'est aussi lui qui constatera pour la première fois la présence de trous dans les papiers originaux et comprendra que la notion de liasse dont parle Étienne dans la Préface de l'édition de 1670 est tout à fait concrète. PaulLouis Couchoud ${ }^{12}$ mettra en évidence en 1948 le classement des papiers en liasses et le fait que la Copie $\left(\mathrm{C}_{1}\right)$ retranscrit les papiers liasse par liasse.

Dans son Introduction à l'édition du Luxembourg (1951), qui suit l'ordre de $C_{1}$, Louis Lafuma n'hésite plus à affirmer que «La Copie 9.203 n'est pas un premier état de l'édition de Port-Royal ; elle est la Copie dont parle Étienne Périer et elle nous donne l'état dans lequel ont été trouvés les papiers de Pascal. » Il considérait aussi que la deuxième Copie $\left(\mathrm{C}_{2}\right)$ n'était qu'une copie de la première. Plutôt que d'une organisation en liasses Lafuma préfère parler de séries de textes, car selon lui, seuls certains papiers ont été mis en liasses.

8 En 1971, Jean Mesnard publie un article ${ }^{13}$ qui constitue une avancée significative dans la connaissance des Copies et le classement effectué par Pascal : il montre, notamment par la méthode des sauts du même au même ${ }^{14}$, que les deux Copies sont issues d'une autre Copie disparue (notée $\mathrm{C}_{0}$ ); il constate aussi que les unités (équivalentes aux séries de textes définies par Lafuma, à la différence près qu'il exclut la séparation des séries classées et des séries non classées) constituent en fait huit grands ensembles cohérents communs aux deux Copies mais ordonnés différemment.

Philippe Sellier (1991) estime que $C_{2}$ présente une bien meilleure organisation en raison de l'hétérogénéité des dossiers ${ }^{15}$ non titrés dans $C_{1}$ : il y distingue d'abord des liasses qui correspondent à la phase préparatoire du travail de Pascal (avant 1658), puis des feuillets qui correspondent au classement effectué par Pascal en juin 1658, et enfin des dossiers de travail qui contiennent des développements postérieurs à 1658. D'autre part, le caractère intangible de la seconde copie l'a convaincu qu'elle était conçue comme « une copie ne varietur ", «la copie de référence, la copie-étalon que les Périer ont fait établir à leur usage, qu'Étienne Périer a constamment améliorée, et souvent en se reportant à l'original ». C'est donc son ordre qu'il a tout naturellement adopté dans ses éditions de 1991,2000 et $2010^{16}$. 
Ordonnancement de $C_{1}$ et $C_{2}{ }^{17}$ (voir le tableau complet en Annexe)

\begin{tabular}{|c|c|}
\hline $\mathrm{C}_{1}$ & $\mathrm{C}_{2}$ \\
\hline $\begin{array}{l}\text { Unités à titres } 1 \text { à } 27 \text { (p. } 1 \text { à } \\
188 \text { ) }\end{array}$ & Table des titres puis unité I (p. 0 à 12 ) \\
\hline Table des titres et unité $\mathrm{I}$ ( $\mathrm{p}$. & Unités à titres 1 à 27 (p. 13 à 220) \\
\hline 189 à 200) & Unité XXXV (p. 221 à 228) \\
\hline Unités II à XIX (p. 201 à 312) & Titre Miracle (p. 225) \\
\hline Unité XX (p. 313 à 316) & Unités XXXII à XXXIV (p. 229 à 274) \\
\hline Unités XXI à XXII (p. 317 à & Unités XXIII à XXXI (p. 275 à 401) \\
\hline 324) & Unités XXI à XXII (p. 401 à 404) \\
\hline Unités XXIII à XXXI (p. 325 à & Unité XX (p. 405 à 410) \\
\hline & Titre Preuves de la religion par le peuple juif, les prophéties et \\
\hline Titre Miracle (p. 431) & quelques discours (p. 409) \\
\hline $\begin{array}{l}\text { Unités XXXII à XXXIV (p. } 435 \text { à } \\
\text { 475) }\end{array}$ & Unités II à XIX (p. 411 à 538) \\
\hline
\end{tabular}

Les grands ensembles dans $C_{2}$

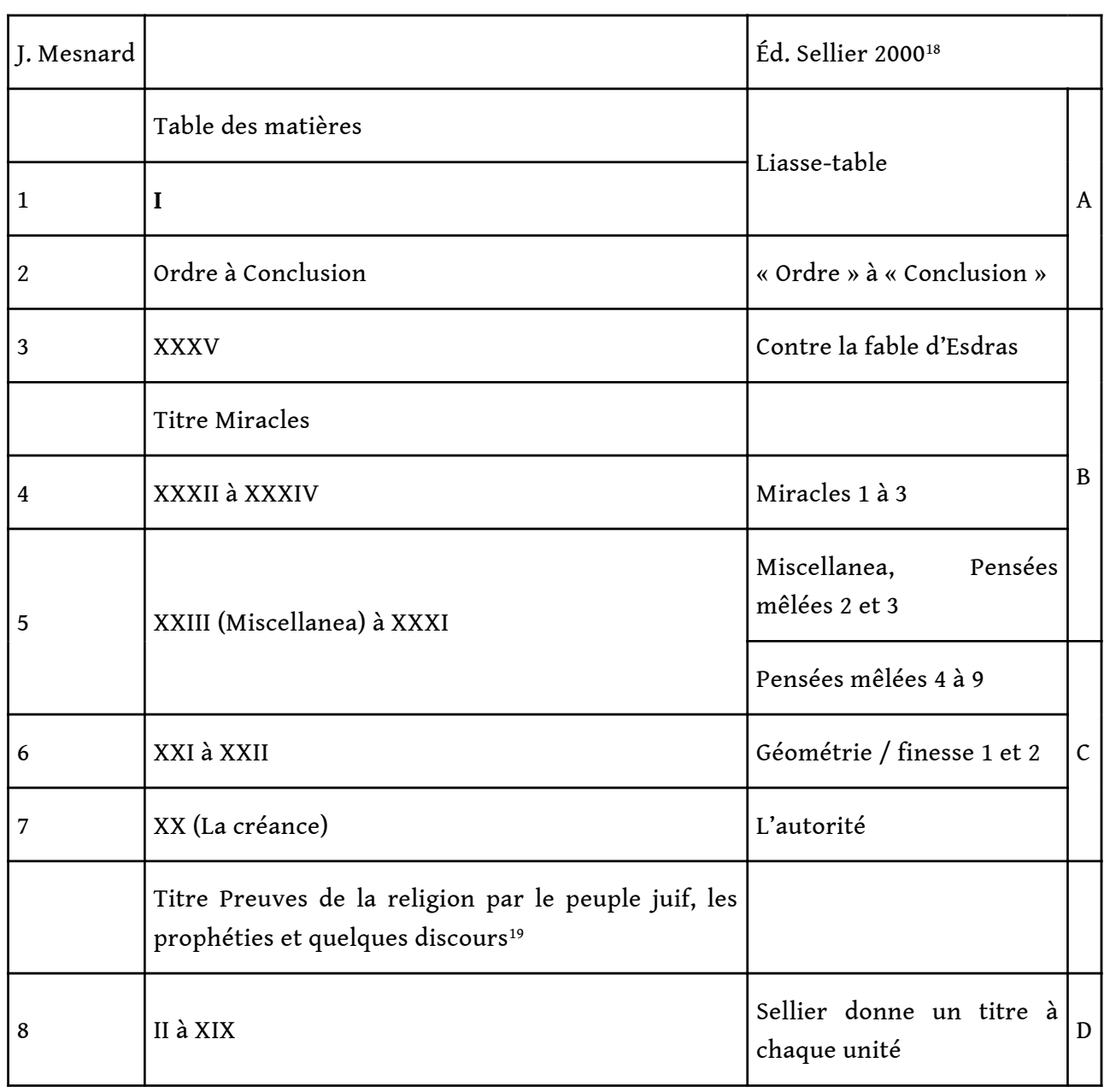


Les conclusions de $\mathrm{J}$. Mesnard sur $\mathrm{C}_{0}$ et les grands dossiers ont permis de constater récemment ${ }^{20}$ que la différence d'ordonnancement entre les deux Copies est plus simple qu'il n'y parait: les grands ensembles qu'il définit constituaient matériellement des dossiers dans $\mathrm{C}_{0}$; ces dossiers ont été entassés par le copiste au fur et à mesure qu'il copiait une première Copie, provoquant un désordre partiel de la transcription de la Copie qui a suivi. Ce processus étant réversible, il ne permet pas de déterminer quelle Copie a été réalisée la première ni de savoir dans quel ordre étaient ces grands ensembles à la mort de Pascal. Cependant, si des unités étaient rassemblées dans des dossiers dans $\mathrm{C}_{0}$ , il resterait à démontrer qu'ils reflètent rigoureusement le classement des papiers par Pascal. En effet, il n'est pas sûr que les deux titres transcrits (voir le tableau ci-dessus) soient de Pascal.

\section{Liasses ou dossiers?}

11 L'étude approfondie des papiers conservés dans le Recueil des originaux permet d'avoir un aperçu significatif de leur organisation en liasses ou en dossiers à la mort de Pascal. Lorsque Pascal voulait enfiler un papier dans une liasse il le perçait en général en haut, à gauche, et si possible dans la marge existante. Certaines feuilles, ainsi que les feuillets séparés, étaient percées en bas, à gauche. Malheureusement, sur beaucoup de ces papiers la marge de gauche a été rognée au moment de leur collage dans le $R O$, ce qui fait que certaines parties trouées ont disparu. De plus, le morceau de papier qui correspondait à l'emplacement du trou n'a pas toujours disparu ${ }^{21}$. Certains de ces minuscules morceaux de papiers ont retrouvé leur place d'origine au moment du collage dans le Recueil et ne sont pas toujours visibles sur les photos en couleur, pourtant d'excellente qualité, sur lesquelles nous pouvons travailler. Seule l'observation minutieuse du manuscrit lui-même permet de les distinguer ${ }^{22}$.

Les tableaux ci-dessous donnent un résumé de nos observations, grand dossier par grand dossier.

1. Unité 1

\begin{tabular}{|l|l|l|l|}
\hline Unité & $\begin{array}{l}\text { Papiers } \\
\text { conservés }\end{array}$ & $\begin{array}{l}\text { Papiers } \\
\text { troués }\end{array}$ & Commentaires \\
\hline I & 32 (3 perdus) & 12 & $\begin{array}{l}\text { Liasse ou papiers provenant de liasses ? le trou d'un des } \\
\text { fragments est déchiré }\end{array}$ \\
\hline
\end{tabular}

\section{Unités à titres}

\begin{tabular}{|l|l|l|l|}
\hline Unité & $\begin{array}{l}\text { Papiers } \\
\text { conservés }^{23}\end{array}$ & $\begin{array}{l}\text { Papiers } \\
\text { troués }^{24}\end{array}$ & Commentaires \\
\hline Ordre & 10 & 3 & \\
\hline
\end{tabular}




\begin{tabular}{|c|c|c|c|}
\hline Vanité & 39 & 13 & $\begin{array}{l}\text { Dont une grande feuille trouée }(50 \mathrm{~cm} \times 38 \mathrm{~cm}) \text { écrite } \\
\text { recto-verso (texte Imagination) et reproduite au } \\
\text { milieu de l'unité }\end{array}$ \\
\hline Misère & 24 & 8 & $\begin{array}{l}\text { Dont un feuillet complet non troué }(25 \mathrm{~cm} \times 37 \mathrm{~cm}) \\
\text { écrit recto-verso (texte Economie du monde) et } \\
\text { reproduit au milieu de l'unité }\end{array}$ \\
\hline Ennui & 3 & 3 & \\
\hline $\begin{array}{l}\text { Raisons des } \\
\text { effets }\end{array}$ & 21 & 6 & \\
\hline Grandeur & 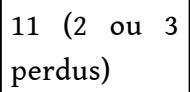 & 5 & \\
\hline Contrariétés & $\begin{array}{ll}12 & (2 \\
\text { perdus })\end{array}$ & 5 & $\begin{array}{l}\text { Dont une feuille trouée }(50 \mathrm{~cm} \text { x } 38 \mathrm{~cm}) \text { écrite recto- } \\
\text { verso (texte contre les pyrrhoniens) et reproduite à } \\
\text { la fin de l'unité }\end{array}$ \\
\hline Divertissement & $6(1$ perdu $)$ & 4 & $\begin{array}{l}\text { Dont le texte Divertissement, composé sur } 5 \text { feuillets } \\
(24 \mathrm{~cm} \times 37 \mathrm{~cm}) \text { écrits uniquement d'un côté, } \\
\text { reproduit au milieu de l'unité ; ces } 5 \text { feuillets ne sont } \\
\text { plus complets et } 3 \text { d'entre eux sont troués }\end{array}$ \\
\hline Philosophes & 7 (1 perdu) & 1 ou 2 & $\begin{array}{l}\text { Dont un des papiers qui semble avoir conservé une } \\
\text { petite trace d'un trou d'enfilage; aucun des papiers } \\
\text { non troués n'a conservé une marge de gauche } \\
\text { complète }\end{array}$ \\
\hline Souverain bien & $1(1$ perdu $)$ & 0 & $\begin{array}{l}\text { Un grand feuillet }(25 \mathrm{~cm} \text { x } 37 \mathrm{~cm}) \text { écrit recto-verso, } \\
\text { rogné à gauche et à droite }\end{array}$ \\
\hline APR & 3 & 3 & Un feuillet, une feuille et un fragment \\
\hline Commencement & 16 & 8 & $\begin{array}{l}\text { Deux des fragments sont troués deux fois; un de ces } \\
\text { trous est déchiré }\end{array}$ \\
\hline Soumission & $22(1$ perdu $)$ & 15 & $\begin{array}{l}\text { Dont un petit papier rectangulaire troué }(6,5 \mathrm{~cm} \times 4,1 \\
\mathrm{cm}) \text { transcrit comme titre de l'unité ; } 5 \text { des fragments } \\
\text { sont troués deux fois }\end{array}$ \\
\hline Excellence & 4 ou $5^{25}$ & 1 ou 2 & $\begin{array}{l}\text { Dont un des papiers qui semble avoir conservé une } \\
\text { petite trace d'un trou d'enfilage; aucun des papiers } \\
\text { non troués n'a conservé une marge de gauche } \\
\text { complète (un doute subsiste pour le papier 151-1) }\end{array}$ \\
\hline Transition & 7 ( 2 perdus) & 3 & $\begin{array}{l}\text { Dont quatre grands feuillets troués }(25 \mathrm{~cm} \text { x } 37 \mathrm{~cm}) \\
\text { écrits recto-verso (texte Disproportion de l'homme) } \\
\text { et reproduits au milieu de l'unité }\end{array}$ \\
\hline
\end{tabular}




\begin{tabular}{|c|c|c|c|}
\hline Fausseté & 18 ou $19^{26}$ & 15 & $\begin{array}{l}\text { Le titre de l'unité (non autographe) a été ajouté à la } \\
\text { sanguine sur le premier papier }\end{array}$ \\
\hline Religion aimable & 2 & 2 & \\
\hline Fondement & 20 & 11 & Un des fragments est troué deux fois \\
\hline Loi figurative & 30 & 17 & $\begin{array}{l}\text { Dont un petit papier rectangulaire troué }(6,9 \mathrm{~cm} \times 3 \\
\mathrm{cm}) \text { transcrit comme titre de l'unité ; un des } \\
\text { fragments est troué deux fois }\end{array}$ \\
\hline Rabbinage & 2 & 2 & \\
\hline Perpétuité & 11 & 5 & Un des fragments est troué deux fois \\
\hline $\begin{array}{l}\text { Preuves de } \\
\text { Moïse }\end{array}$ & 7 & 3 & \\
\hline Preuves de J.C. & 24 & 6 & \\
\hline Prophéties & $\begin{array}{l}24 \\
\text { perdus })\end{array}$ & 7 & \\
\hline $\begin{array}{l}\text { Figures } \\
\text { particulières }\end{array}$ & 2 & 0 & Les deux papiers ont été découpés très près du texte \\
\hline $\begin{array}{l}\text { Morale } \\
\text { chrétienne }\end{array}$ & $\begin{array}{ll}23 & (2 \\
\text { perdus }) & \end{array}$ & 5 & \\
\hline Conclusion & 6 & 3 & \\
\hline
\end{tabular}

Ces résultats confirment que tous ces papiers pouvaient être regroupés dans des liasses.

Plusieurs de ces liasses, notamment Vanité et Misère, sont composées surtout de petits papiers et d'un nombre variable de feuillets. On aurait pu s'attendre à ce que les grands feuillets soient stockés au-dessous, et transcrits après les petits papiers. Il n'en est rien. Cela est d'autant plus surprenant dans le cas du texte sur l'Économie du monde (Sel. 94) qui a été écrit en partie sur un feuillet resté intact et non troué ${ }^{27}$.

\section{Unité XXXV}

\begin{tabular}{|l|l|l|l|}
\hline Unité & $\begin{array}{l}\text { Papiers } \\
\text { conservés }\end{array}$ & $\begin{array}{l}\text { Papiers } \\
\text { troués }\end{array}$ & Commentaires \\
\hline XXXV & 4 & 2 & $\begin{array}{l}\text { La marge de gauche des deux papiers non troués n'a pas été } \\
\text { conservée ; la transcription de ces papiers est absente de } C_{1}\end{array}$ \\
\hline
\end{tabular}




\begin{tabular}{|l|l|l|l|}
\hline XXXII & aucun & & Correspondrait environ à une feuille dont un feuillet écrit $\mathrm{r} / \mathrm{v}$ \\
\hline XXXIII & 17 & 14 & $\begin{array}{l}\text { Une liasse constituée surtout de feuillets complets provenant de feuilles (35 } \\
\mathrm{cm} \text { X } 25,5 \mathrm{~cm}) \text { découpées par Pascal ou après sa mort }\end{array}$ \\
\hline XXXIV & 11 & 11 & $\begin{array}{l}\text { Une liasse constituée de papiers de tailles et de provenances diverses dont } 6 \\
\text { portent un texte d'abord écrit au crayon }\end{array}$ \\
\hline
\end{tabular}

5. Pensées diverses (Miscellanea ?)

\begin{tabular}{|c|c|c|c|}
\hline XXIII & $\begin{array}{ll}35 & (2 \\
\text { perdus })\end{array}$ & 20 & $\begin{array}{l}\text { Ces papiers étaient probablement réunis dans une liasse; un papier } \\
\text { est percé de deux trous }\end{array}$ \\
\hline XXIV & $\begin{array}{ll}32 & (5 \\
\text { perdus })\end{array}$ & 15 & $\begin{array}{l}\text { Ces papiers étaient probablement réunis dans une liasse; deux } \\
\text { papiers sont percés de deux trous ; quatre papiers sont aussi percés de } \\
\text { trous d'épingle }\end{array}$ \\
\hline XXV & $\begin{array}{lr}78 & (\mathrm{au} \\
\text { moins } & 5 \\
\text { perdus }) & \end{array}$ & 0 & $\begin{array}{l}\text { Pol Ernst a montré que ces papiers ont été découpés lors du collage } \\
\text { dans le Recueil et proviennent de } 19 \text { feuillets (ou } 10 \text { feuilles } 43 \mathrm{~cm} \text { x } \\
33,5 \mathrm{~cm} \text { ) écrits uniquement d'un côté. }\end{array}$ \\
\hline XXVI & $\begin{array}{ll}21 & (2 \\
\text { perdus })\end{array}$ & 6 & $\begin{array}{l}\text { Ces papiers étaient probablement réunis dans une liasse ; } 7 \text { papiers } \\
\text { (dont } 4 \text { écrits recto-verso) portent l'écriture de Gilberte Périer et } 2 \\
\text { papiers portent quelques mots d'illettrés; un de ces papiers est percé } \\
\text { de deux trous }\end{array}$ \\
\hline XXVII & 7 & 2 & $\begin{array}{l}\text { Un papier porte l'écriture de Gilberte ; aucun des papiers non troués } \\
\text { n'a conservé une marge de gauche complète }\end{array}$ \\
\hline XXVIII & 5 & 1 & $\begin{array}{l}\text { Aucun des papiers non troués n'a conservé une marge de gauche } \\
\text { complète }\end{array}$ \\
\hline XXIX & 10 & 5 & $\begin{array}{l}\text { Ces papiers étaient probablement réunis dans une liasse; deux des } \\
\text { papiers sont percés de deux trous }\end{array}$ \\
\hline XXX & 6 & 2 & $\begin{array}{l}\text { La reconstitution de Pol Ernst (Album, p. 184) montre que le papier } \\
159-3 \text { ne portait pas de trou d'enfilage }\end{array}$ \\
\hline XXXI & 2 & 2 & $\begin{array}{l}\text { Correspondait à une feuille }(35 \mathrm{~cm} \text { x } 27,5 \mathrm{~cm}) \text { écrite uniquement d'un } \\
\text { côté }\end{array}$ \\
\hline
\end{tabular}

\section{Unités XXI-XXII}

\begin{tabular}{|l|l|l|l|}
\hline XXI & 1 & 0 & Un feuillet écrit d'un côté \\
\hline XXII & 2 & 0 & \\
\hline
\end{tabular}




\section{Unité XX}

\begin{tabular}{|l|l|l|l|}
\hline $\mathrm{XX}$ & 8 & 0 & $\begin{array}{l}\text { Correspondait à une feuille }(43 \mathrm{~cm} \times 31,5 \mathrm{~cm}) \text { écrite d'un seul côté. Pol Ernst pense } \\
\text { que cette feuille a été découpée lors du collage des papiers dans le RO, ce qui reste à } \\
\text { démontrer }\end{array}$ \\
\hline
\end{tabular}

\section{Preuves de la religion par le peuple juif*, les prophéties* ${ }^{\star \star}$ et quelques discours ${ }^{\star \star \star}$}

\begin{tabular}{|c|c|c|c|}
\hline Unité & $\begin{array}{l}\text { Papiers } \\
\text { conservés }\end{array}$ & $\begin{array}{l}\text { Papiers } \\
\text { troués }\end{array}$ & Commentaires \\
\hline II $* * *$ & 3 & 0 & $\begin{array}{l}\text { Un demi-feuillet et un papier plié en } 4 \text { provenant probablement } \\
\text { d'une feuille écrite recto-verso }(47 \mathrm{~cm} \times 37,5 \mathrm{~cm})\end{array}$ \\
\hline III*** & aucun & & Correspondrait environ à une feuille et demie écrite recto-verso \\
\hline $\mathrm{IV}^{* * *}$ & aucun & & Correspondrait à un feuillet écrit uniquement d'un côtée 28 \\
\hline $\mathrm{V}^{* * *}$ & aucun & & $\begin{array}{l}\text { Correspondrait environ à une feuille dont un feuillet écrit } \\
\text { recto-verso }\end{array}$ \\
\hline VI* & 2 & 0 & Deux feuillets dont un qui est écrit recto-verso \\
\hline VII* & 1 & 0 & $\begin{array}{l}\text { Feuillet rogné écrit d'un côté et issu d'une feuille ( } 47 \mathrm{~cm} \times 37,5 \\
\mathrm{~cm})\end{array}$ \\
\hline VIII* & 1 & 0 & Une feuille $(43 \mathrm{~cm} \times 33,5 \mathrm{~cm})$ écrite recto-verso \\
\hline $\mathrm{IX}^{*}$ & 1 & 0 & $\begin{array}{l}\text { Un feuillet et demi écrit en partie recto-verso et probablement } \\
\text { issu d'une grande feuille }(50 \mathrm{~cm} \times 38 \mathrm{~cm})\end{array}$ \\
\hline $\mathrm{X}^{*}$ & 1 & 1 & Papier isolé qui a probablement transité par une liasse \\
\hline $\mathrm{XI}^{*}$ & $3(+$ pertes $)$ & 0 & L'équivalent d'un feuillet écrit recto-verso est perdu \\
\hline XII* & aucun & & $\begin{array}{l}\text { Correspondrait environ à une feuille dont un feuillet écrit } \\
\text { recto-verso }\end{array}$ \\
\hline XIII** & 2 & 0 & $\begin{array}{l}\text { Probablement une feuille }(47 \mathrm{~cm} \times 37,5 \mathrm{~cm}) \text { dont un feuillet } \\
\text { écrit recto-verso }\end{array}$ \\
\hline $\mathrm{XIV}^{* *}$ & 16 & 0 & $\begin{array}{l}\text { Correspondait probablement à } 11 \text { feuillets écrits uniquement } \\
\text { d'un côté ( } 5 \text { feuilles } 1 / 238 \mathrm{~cm} \mathrm{x} 29,5 \mathrm{~cm} \text { ?) et découpés au } \\
\text { moment du collage dans le Recueil }\end{array}$ \\
\hline $\mathrm{XV}^{* *}$ & 14 & 0 & $\begin{array}{l}\text { Correspondait probablement à } 8 \text { feuillets écrits uniquement } \\
\text { d'un côté ( } 4 \text { feuilles } 35 \mathrm{~cm} \text { x 25,5 cm ?). Pol Ernst pensait que le } \\
\text { découpage avait eu lieu au moment du collage dans le Recueil, } \\
\text { ce qui n'est pas démontré }\end{array}$ \\
\hline
\end{tabular}




\begin{tabular}{|l|l|l|l|}
\hline XVI** & 1 & 1 & $\begin{array}{l}\text { Un feuillet écrit d'un côté, qui a probablement transité par une } \\
\text { liasse (comme l'unité X) }\end{array}$ \\
\hline XVII** & 10 & 0 & $\begin{array}{l}10 \text { feuillets complets qui correspondaient à } 5 \text { feuilles ( } 47 \mathrm{~cm} \times \\
37,5 \mathrm{~cm}) \text { écrites uniquement d'un côté et dont les feuillets ont } \\
\text { été séparés lors de leur montage dans le } R 0\end{array}$ \\
\hline XVIII** & 1 & 0 & Un feuillet écrit d'un côté \\
\hline XIX** & 5 & 3 ou 4 & $\begin{array}{l}\text { Correspondait à } 5 \text { feuillets écrits uniquement d'un côté (dont } \\
\text { deux feuilles } 35 \mathrm{~cm} x 25,5 \mathrm{~cm} \text { et un feuillet } 23,5 \mathrm{~cm} \mathrm{x} 37,5 \mathrm{~cm}) ; \\
\text { un feuillet est percé de deux trous ; un des papiers semble avoir } \\
\text { conservé une trace d'un trou d'enfilage ; le premier papier a } \\
\text { conservé la partie supérieure de sa marge de gauche et aucun } \\
\text { trou n'y est visible }\end{array}$ \\
\hline
\end{tabular}

\section{Conclusions}

Les unités titrées étaient composées de liasses et peut-être de feuilles ou de feuillets indépendants. Ces documents étaient probablement stockés dans des tiroirs ou sur des étagères, ou les deux à la fois ${ }^{29}$. Ainsi on ne peut pas exclure qu'un papier laissé libre se soit glissé au milieu d'une liasse ou que deux liasses, transcrites en une seule dans les Copies, étaient stockées dans un même tiroir. Il est curieux que sur les 27 liasses titrées seuls deux petits papiers qui pouvaient servir d'étiquettes aient été retrouvés.

Pour les autres grands dossiers l'organisation était plus complexe. Chaque grand dossier pouvait contenir soit des feuilles doubles et/ou des feuillets séparés, soit des papiers enfilés en liasses, soit les deux à la fois ${ }^{30}$.

Peut-on supposer que les papiers ont d'abord été stockés dans une première liasse d'attente avant d'être classés dans les liasses où ils ont été trouvés? Dans cette éventualité les trous qu'on peut constater sur les papiers ne seraient que les témoins d'un premier enfilage et non la preuve qu'ils étaient restés enfilés. Étienne Périer répond en partie dans la Préface de l'édition: "On les trouva tous ensemble enfilez en diverses liasses ». Il dit bien enfilés et non simplement entassés.

D'autre part, l'observation des papiers nous a permis de faire une petite découverte qui révèle assez bien le travail de découpage et de constitution des liasses par Pascal : au verso du papier 255-2 (Liasse Perpétuité, Sel. 321, Laf. 289) ${ }^{31}$, dont le texte est transcrit en tête de la liasse, est inscrit le mot «Aymable » (visible en transparence). $Z$. Tourneur avait découvert qu'au verso du papier 227-4 (Liasse Religion aimable, Sel. 255, Laf. 222) ${ }^{32}$, dont le texte est aussi transcrit en tête de la liasse, est inscrit le renvoi « voyez Perpetuités ${ }^{33}$ ». Ces deux textes se rapportent au même sujet (les Juifs charnels et les païens). Pol Ernst (Album , p. 165) avait émis l'hypothèse que ces deux papiers provenaient d'un même feuillet avant d'être découpés par Pascal. Il se fondait notamment sur le fait que ces deux papiers portent l'écriture du «secrétaire assidu» de Pascal. Il est vraisemblable que Pascal a voulu garder un lien entre ces deux papiers au moment où il créait les deux liasses ${ }^{34}$. 


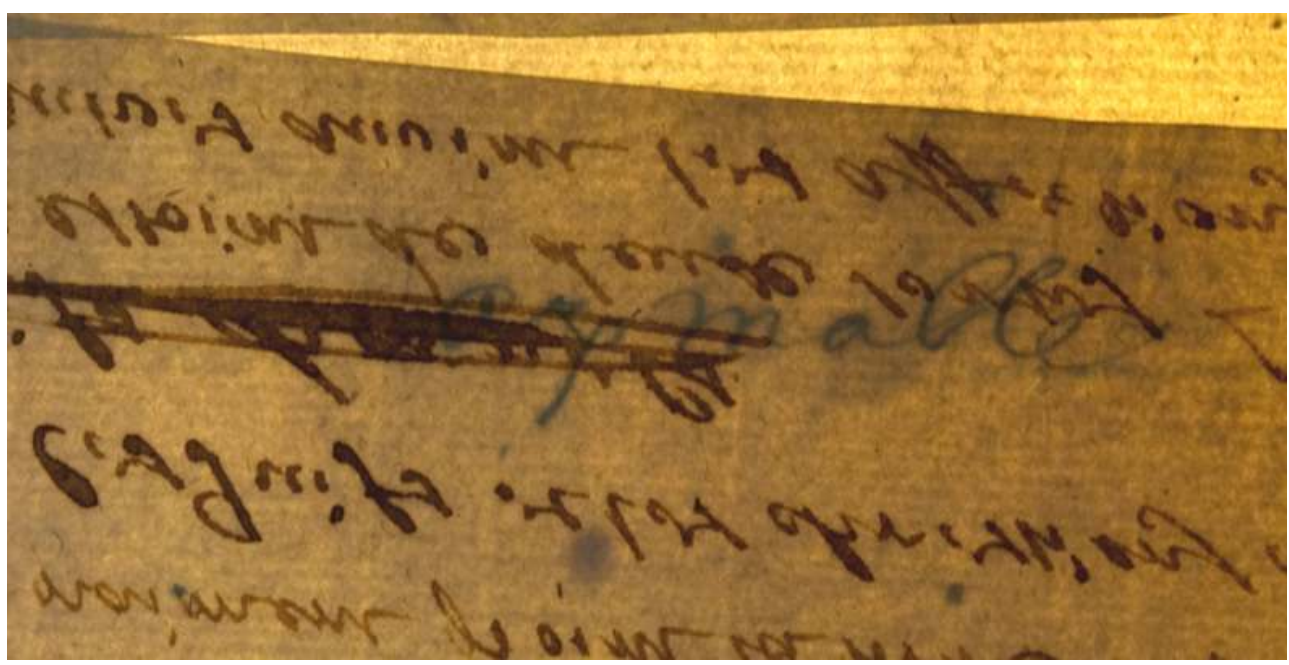

227-4 (Rendre la religion aimable) : « voyez perpétuité 》

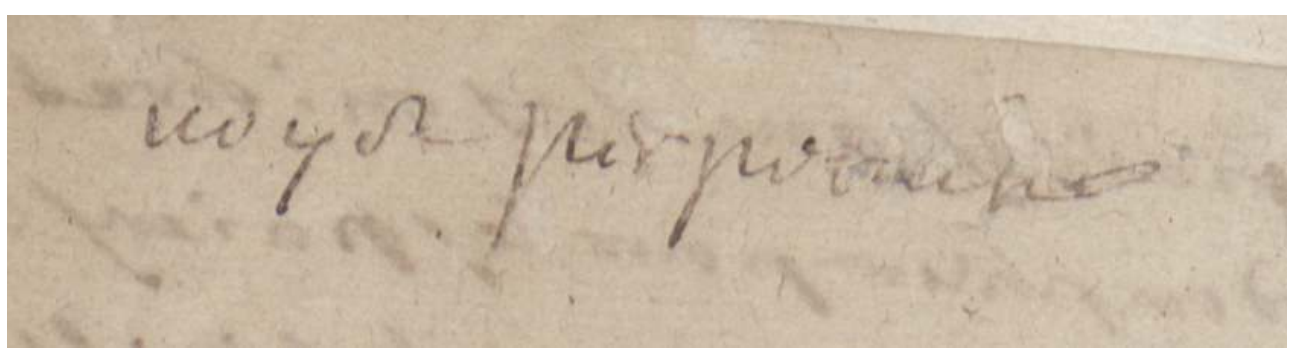

\section{Première partie : confection des Copies}

\section{Description matérielle}

La Copie $\mathrm{C}_{1}$ est un volume de 472 pages écrites recto-verso (paginées de 1 à 472) encadrées par 3 feuilles doubles et composé de 62 cahiers de taille variable (à la fois dans le nombre de feuilles doubles contenues dans ces cahiers et dans la dimension des feuillets : la hauteur varie actuellement de 34,2 à $35,1 \mathrm{~cm}$ et la largeur de 22,1 à $23 \mathrm{~cm}$ ). Chaque cahier correspond à la transcription d'une unité (liasse ou dossier).

Il n'est plus possible de donner une description matérielle complète du volume d'origine : en effet, la reliure a été refaite en 1970 pour renforcer la base des feuillets. La couverture et les pages de garde ont été remplacées par des pages récentes et ne semblent pas avoir été conservées.

Z. Tourneur, dans l'Introduction de son Édition paléographique en 1942 (p. 13), a donné des informations qui permettent de compléter l'observation qu'on peut en faire aujourd'hui : «Longtemps restés à l'état de brochures, comme le montre la couleur de la première page, ces cahiers ont été reliés, après 1731, entre deux feuilles au filigrane de Martel, Limosin, avec une couverture en papier vert sur simple carton, sans tranches. Le dos porte la mention Copie des Pensées de Pascal, sans date. » L. Lafuma rectifiera cette information dans les Recherches pascaliennes en 1949 (p. 33, note 24) en signalant que le filigrane de la page de garde était en fait MABARET LIMOSIN. Ce filigrane correspond à un propriétaire- 
marchand ${ }^{35}$ de la région de Saint-Léonard-de-Noblat (Haute-Vienne) et ne permet pas, dans l'état de nos connaissances, de savoir à quelle période précise il a été utilisé. Nous constatons cependant que la couverture était de la même couleur que celle du Recueil des originaux $^{36}$ et que ces deux documents ont été conservés ensemble à la Bibliothèque Royale.

Autre conséquence malheureuse de ce nécessaire travail de réfection: la reliure très serrée rend difficile la lecture à l'intérieur de celle-ci et le renfort opaque ajouté à l'intérieur des feuillets occulte les dernières lettres du texte, ce qui empêche de photographier entièrement les feuillets.

Observations faites récemment sur le manuscrit:

- Le titre COPIE / DES PEN / DE PASC est écrit sur la tranche en lettres dorées sur fond rouge.

- Une étiquette blanche a été collée sur la tranche et porte la référence FR 9203. La même étiquette a été collée sur la première page de couverture.

Copie $\mathrm{C}_{1}$

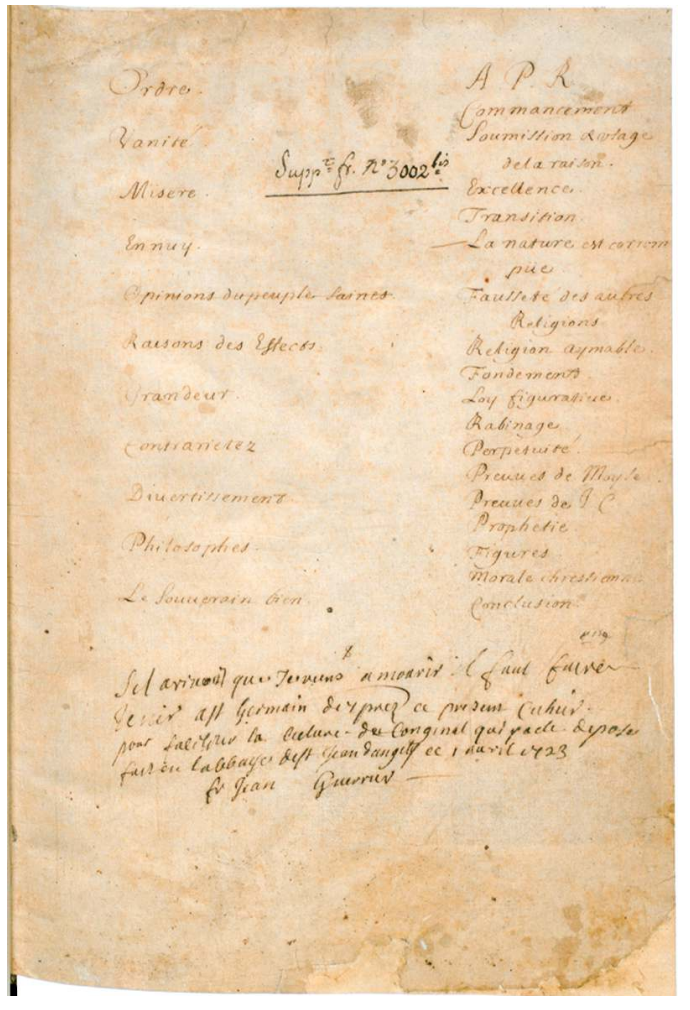

BNF

La première page de couverture et les trois premiers feuillets sont récents. Ils ont été rajoutés lors de la restauration pour équilibrer le volume et remplacer les anciennes pages de garde.

Le reste est plus ancien et composé de deux ensembles de cahiers qui commencent chacun par une table de 29 titres présentés sur deux colonnes de même longueur mais de largeur différentes. Le titre Opinions du peuple saines est barré et le titre La nature est corrompue est signalé par un trait uniquement sur la première table. Cette première table contient l'ancienne référence de l'ouvrage ainsi que le texte manuscrit, de la main de Jean Guerrier : 
S'il arivait que je viens a mourir il faut faire

Venir a st Germain des prez ce present Cahier

Pour faciliter la lecture de loriginal qui y a été depose

Fait en labbaye de st Jean d'angely ce 1 avril 1723

Fr Jean Guerrier ${ }^{37}$

Le numéro de page de la deuxième table a été ajouté en tous petits caractères (V. 189) audessus de ce texte $\mathrm{e}^{38}$.

Chaque exemplaire de la table est terminé par un S barré suivi d'un grand trait final.

Chaque cahier a été constitué de feuilles pliées en deux et imbriquées les unes dans les autres. Tous les cahiers sont constitués de feuilles provenant d'un seul type de papier sauf celui du dossier $\mathrm{V}$ qui est composé de deux feuilles de deux types différents mais de même origine (voir le tableau ci-dessous).

Filigranes (marques ${ }^{39} \&$ contremarques) des papiers : 7 types de feuilles de dimensions très proches (env. $45 \grave{a} 46 \mathrm{~cm} \times 35 \mathrm{~cm})^{40}$

1. Armes des Médicis (dim. L x H :6,8 cm x $9 \mathrm{~cm}$ ) \& grappe de raisin soutenant les lettres PC/L présentées dans un grand cœur (dim. 2,6 cm x 5,6 cm): 65 feuilles.

2. Armes des Médicis \& C [Trèfle à 4 feuilles ?] I dans un cartouche soutenant une grappe de raisin et surmonté d'une couronne royale $(\operatorname{dim} .5,9 \mathrm{~cm} \times 2,2 \mathrm{~cm})^{41}$ : une feuille.

3. Raisin (dim. : 1,9 cm x $3 \mathrm{~cm}$ ) \& B RODIER dans un cartouche (dim. $6 \mathrm{~cm} \times 0,9 \mathrm{~cm}): 13$ feuilles.

4. Écu 3 annelets simples entouré de lauriers (dim. $7,3 \mathrm{~cm} \mathrm{x} \mathrm{8,8} \mathrm{cm)} \mathrm{\&} \mathrm{P} \mathrm{cœur} \mathrm{V} \mathrm{dans} \mathrm{un}$ cartouche parallèle aux pontuseaux $(\operatorname{dim} .3 \mathrm{~cm} \times 1,1 \mathrm{~cm}):$ deux feuilles.

5. Armes d'un cardinal ou archevêque soutenant un écu couronné (couronne d'un vicomte ou d'un marquis) et les lettres PC/L mises dans un grand cœur (filigrane unique - dim. 11,5 cm x $11,5 \mathrm{~cm}): 16$ feuilles $^{42}$.

6. Armes d'un prélat, différentes du type 5 , soutenant un écu non couronné et les lettres PC/L mises dans un grand cœur (filigrane unique - dim. 9,5 cm x $7 \mathrm{~cm}$ ) : 9 feuilles.

7. Armes des Médicis \& A cœur F (ou A cœur P ?) dans un cartouche parallèle aux pontuseaux (dim. 2,9 cm x $1 \mathrm{~cm}): 13$ feuilles.

\begin{tabular}{|c|c|c|c|}
\hline Liasse & Type de filigrane & Dossier & Type de filigrane \\
\hline Table des titres & 1 (1 feuille) & \multirow{2}{*}{$\begin{array}{l}\text { Table des titres } \\
+ \text { I }\end{array}$} & \multirow{2}{*}{1 (3 feuilles) } \\
\hline Ordre & 3 (1 feuille) & & \\
\hline Vanité & 3 (3 feuilles) & II & 1 (2 feuilles) \\
\hline Misère & 5 (3 feuilles) & III & 1 (3 feuilles) \\
\hline Ennui & 3 (1 feuille) & IV & 6 (1 feuille) \\
\hline Raison des effets & 3 (2 feuilles) & $\mathrm{V}$ & 6 (1 feuille), 5 ( 1 feuille) \\
\hline Grandeur & 2 (2 feuilles) & VI & 1 (1 feuille) \\
\hline Contrariétés & 3 (2 feuilles) & VII & 6 (1 feuille) \\
\hline Divertissement & 3 (2 feuilles) & VIII & 7 (1 feuille) \\
\hline
\end{tabular}




\begin{tabular}{|c|c|c|c|}
\hline Philosophes & 3 (1 feuille) & IX & 6 (1 feuille) \\
\hline Souverain bien & 5 (1 feuille) & $\mathrm{X}$ & 6 (1 feuille) \\
\hline APR & 1 (2 feuilles) & XI & 1 (2 feuilles) \\
\hline Commencement & 1 (1 feuille) & XII & 7 (2 feuilles) \\
\hline Soumission & 1 (1 feuille) & XIII & 6 (1 feuille) \\
\hline Excellence & 1 (1 feuille) & XIV & 1 (2 feuilles) \\
\hline Transition & 1 (4 feuilles) & $\mathrm{XV}$ & 1 (2 feuilles) \\
\hline Fausseté & 1 (2 feuilles) & XVI & 1 (1 feuille) \\
\hline Religion aimable & 1 (1 feuille) & XVII & 1 (3 feuilles) \\
\hline Fondements & 1 (2 feuilles) & XVIII & 5 (1 feuille) \\
\hline Loi figurative & 1 (4 feuilles) & XIX & 1 (2 feuilles) \\
\hline Rabbinage & 1 (1 feuille) & $x x$ & 1 (1 feuille) \\
\hline Perpétuité & 4 (2 feuilles) & XXI & 6 (1 feuille) \\
\hline Preuves de Moïse & 1 (1 feuille) & XXII & 6 (1 feuille) \\
\hline Preuves de J.-C. & 1 (2 feuilles) & XXIII & 7 (6 feuilles) \\
\hline Prophéties & 1 (2 feuilles) & XXIV & 5 (4 feuilles) \\
\hline Figures particulières & 3 (1 feuille) & $\mathrm{XXV}$ & 1 (5 feuilles) \\
\hline Morale chrétienne & 1 (2 feuilles) & XXVI & 7 (4 feuilles) \\
\hline \multirow[t]{8}{*}{ Conclusion } & 1 (1 feuille) & XXVII & 5 (2 feuilles) \\
\hline & & XXVIII & 1 (2 feuilles) \\
\hline & & XXIX & 1 (2 feuilles) \\
\hline & & $\mathrm{XxX}$ & 1 (1 feuille) \\
\hline & & XXXI & 1 (1 feuille) \\
\hline & & XXXII & 6 (1 feuille) \\
\hline & & XXXIII & 5 (4 feuilles) \\
\hline & & XXXIV & 1 (4 feuilles) \\
\hline
\end{tabular}


urs de ces filigranes sont de toute évidence contemporains de ceux qui ont été observés sur les papiers utilisés par Blaise Pascal et certains proviennent des mêmes papetiers. C'est le cas de la contremarque PC/L qu'on retrouve sous un filigrane IHS dans un soleil ${ }^{43}$ (dont les papiers du RO correspondent tous au dossier XIV - Prophéties) ; de B RODIER qu'on retrouve dans le filigrane Écu 3 annelets doubles / PF et pot / B RODIER (dont les papiers du RO correspondent à de nombreux papiers de diverses liasses) ; enfin, des armes des Médicis dont on retrouve une variante sur trois ou quatre papiers du RO et l'Écu 3 annelets simples (sans PF).

Ces filigranes attestent que la Copie $C_{1}$ a été réalisée au maximum quelques années après la mort de Pascal.

Le tableau montre aussi que, les feuilles étant imbriquées les unes dans les autres, la deuxième table des titres (p. 189) a été écrite sur la même feuille que le dernier feuillet du dossier I.

Un feuillet écrit uniquement d'un côté mais plié en deux a été ajouté dans la Copie p. 154 entre deux feuillets vierges situés à la fin du cahier Preuves de Moïse. ${ }^{44}$

Feuillet original dans la copie $\mathrm{C}_{1}$

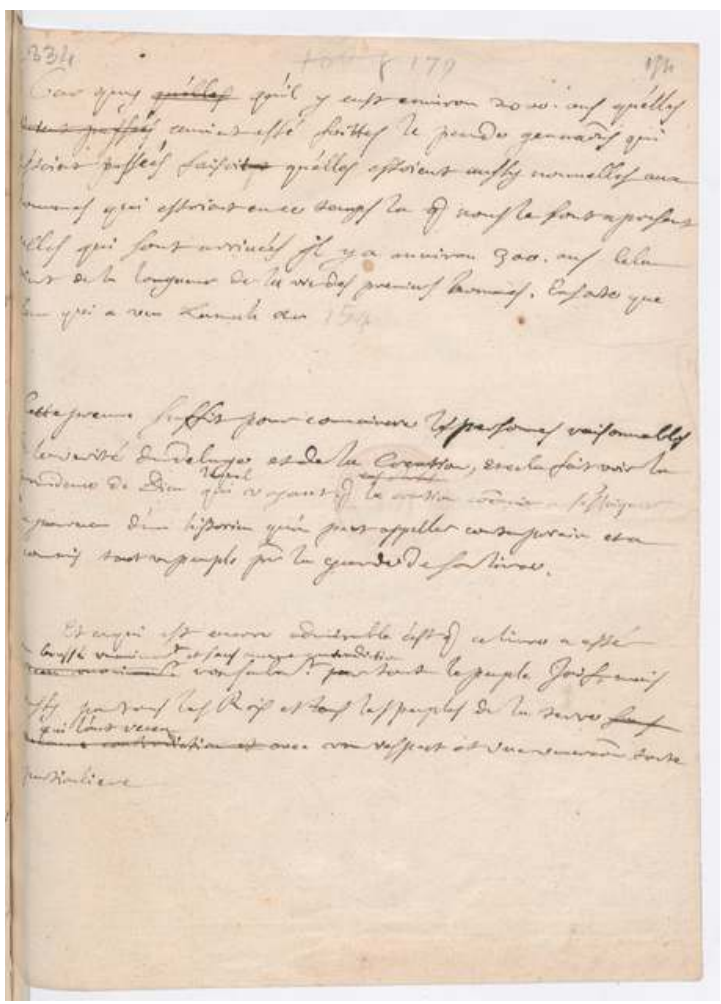

* L. Brunschvicg ( $n^{\circ} 624$, note 3, p. 67) y voyait l'écriture du duc de Roannez et le présentait comme un grand commentaire où Gilberte ne reconnaissait plus l'œuvre de son frère ; L. Lafuma (note du n ${ }^{\circ} 292$ ) disait que c'était l'écriture de Nicole. Ce texte n'est-il pas « trop » lisible pour être de Pascal ? Ne pourrait-il pas être un essai de première transcription d'un document écrit par Pascal ${ }^{45}$ ?

(BNF)

Le fragment (Sel. 741) serait, selon J. Mesnard (Les « Pensées » de Pascal ont trois cent ans, p. 11)*, un autographe de Pascal ajouté tardivement à $C_{1}$ pour l'illustrer. Le feuillet, coupé très droit, pourrait provenir d'un feuillet vierge d'un livre imprimé. Il porte un fragment 
de filigrane deux écus Lion et fleurette quintefeuille surmontés d'une couronne (dim. 6,8 $\mathrm{cm} \times 5,2 \mathrm{~cm}$ ). Le texte n'a pas son équivalent dans le $R O$.

Dessin du fragment de filigrane

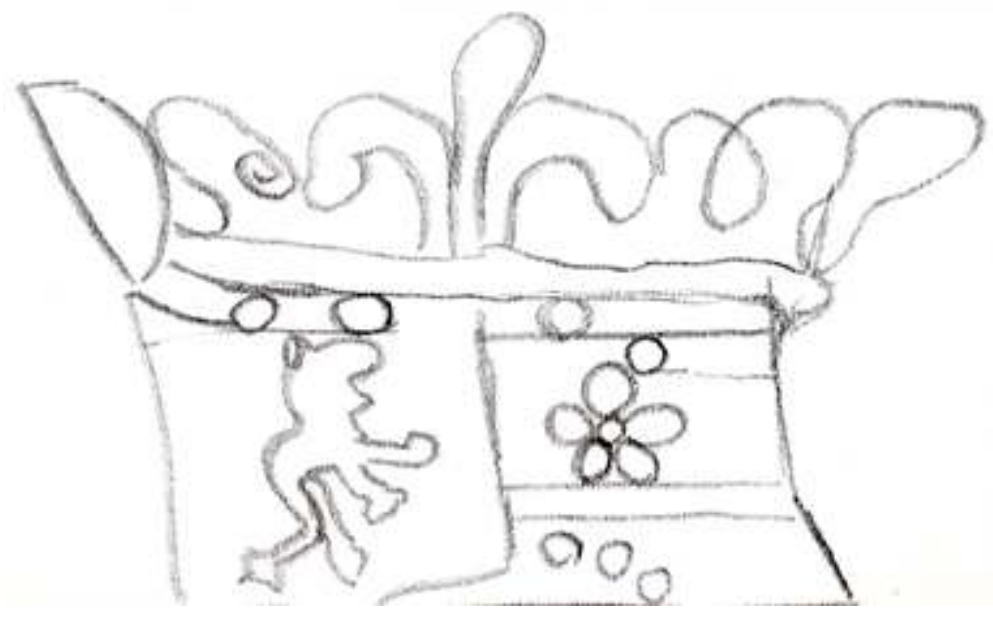

$(\mathrm{BNF})$

Le Recueil $\mathbf{R C}_{2}{ }^{46}$ est un gros volume in-folio de $22 \mathrm{~cm}$ de large et $33 \mathrm{~cm}$ de haut, composé de 922 pages (suivies de deux pages de garde) réparties dans 86 cahiers de tailles diverses. La plupart des pièces ont été massicotées et seuls quelques numéros de cahiers ont été préservés. D’après J. Mesnard (OC I, p. 294) «Ce volume a été formé par l'assemblage de cahiers ou groupes de cahiers primitivement indépendants. Beaucoup de ces cahiers portent, au bas de la première page, de la main du P. Guerrier, un numéro. Certains de ces numéros ont été rognés à la reliure et sont devenus peu lisibles. D'autres ont sans doute complètement disparu. D’autres n'ont jamais été portés ».

«[...] La reliure primitive était en veau du XVIII ${ }^{e}$ siècle ». Si les reliures de la Copie $C_{1}$ et du Recueil des originaux ont peut-être été réalisées par un même relieur, il semble donc que ce ne soit pas le cas pour $\mathrm{RC}_{2}$.

La reliure a été refaite récemment en même temps que celle de $C_{1}$. Elle porte au dos le titre Recueil. Les quatre onglets des pages 419 à 426 (cahier 30) ont été refaits; la feuille paginée 399-400-401-402 (cahier 28), et les feuillets 403-404, 417-418 (cahier 29) et 427-428 (cahier 31) ont été montés sur onglet.

La Copie $\mathrm{C}_{2}$ ne constitue qu'une partie du Recueil $\mathrm{RC}_{2}$. Elle commence par une liste de titres semblable à celles de $C_{1}$, suivie de la page 1 , et se termine à la page 538 (les pages 532 à 538 sont blanches). $C_{2}$ est suivie d'un cahier paginé 539 à 552 qui porte un texte intitulé Que la Loy estoit figurative. Ce cahier, sur lequel nous reviendrons plus loin, ne fait pas partie de $\mathrm{C}_{2}$.

La Copie $C_{2}$ est composée de 135 feuilles réparties dans 35 cahiers, anciennement numérotés de 3 à 37, et contenant chacun 4 feuilles doubles (16 pages) imbriquées les unes dans les autres, exception faite du cahier 28 et du cahier 30 (deux feuilles doubles). Les quatre feuillets paginés 419 à 426 du cahier 30 étaient, selon J. Mesnard, montés sur quatre onglets. Le cahier 28 est particulier: il est composé d'une feuille double sans filigrane et porte l'écriture de Pierre Guerrier au lieu de celle du copiste habituel ${ }^{47}$. 
La plupart des unités (liasses et dossiers) ont été écrites sans tenir compte des changements de cahiers. La majorité des feuillets sont écrits recto-verso sauf quelques pages laissées blanches de façon quasi anarchique. La Copie ne contient qu'un seul exemplaire de la table des titres : cette table est écrite sur un feuillet coté B et porte la signature de Pierre Guerrier; le verso est resté vierge; la présentation des titres est, comme dans $\mathrm{C} 1$, sur deux colonnes mais de longueurs et largeurs différentes. Chaque colonne est terminée par un $\mathrm{S}$ barré. Le titre Raisons des effets a été ajouté par un réviseur dans l'interligne laissé par le copiste entre Opinions du peuple saines et Grandeur. Rien ne signale le titre La nature est corrompue ${ }^{48}$; l'ensemble est terminé comme dans $\mathrm{C} 1$ par un $\mathrm{S}$ barré et un trait de soulignement.

41 Tous ces cahiers, sauf le cahier 28, portent un même filigrane, constitué de 3 fleurs de lys surmontées de 3 pendrillons dans un losange surmonté d'une couronne, et entouré de lauriers, soutenant les lettres $\mathrm{PB} \cdot \mathrm{AB}$ (ou PR•AB ?) dans un cartouche ( $\mathrm{L} x \mathrm{H}: 6,5 \mathrm{~cm} \times 7,9$ $\mathrm{cm}$ ). Ce filigrane est unique (un feuillet sur deux) et n'a pas d'équivalent sur les papiers de Pascal.

\section{Filigrane}

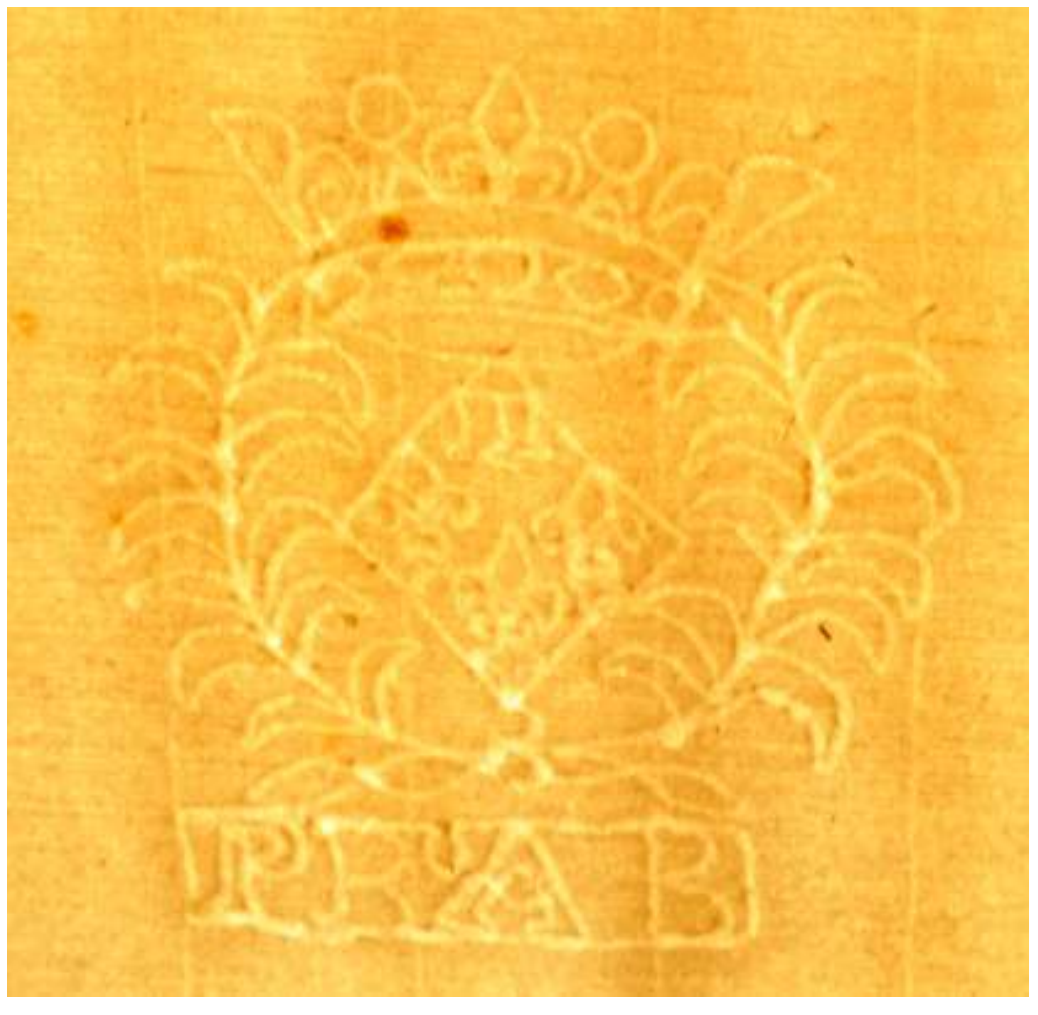

BNF 
Copie $\mathrm{C}_{2}$

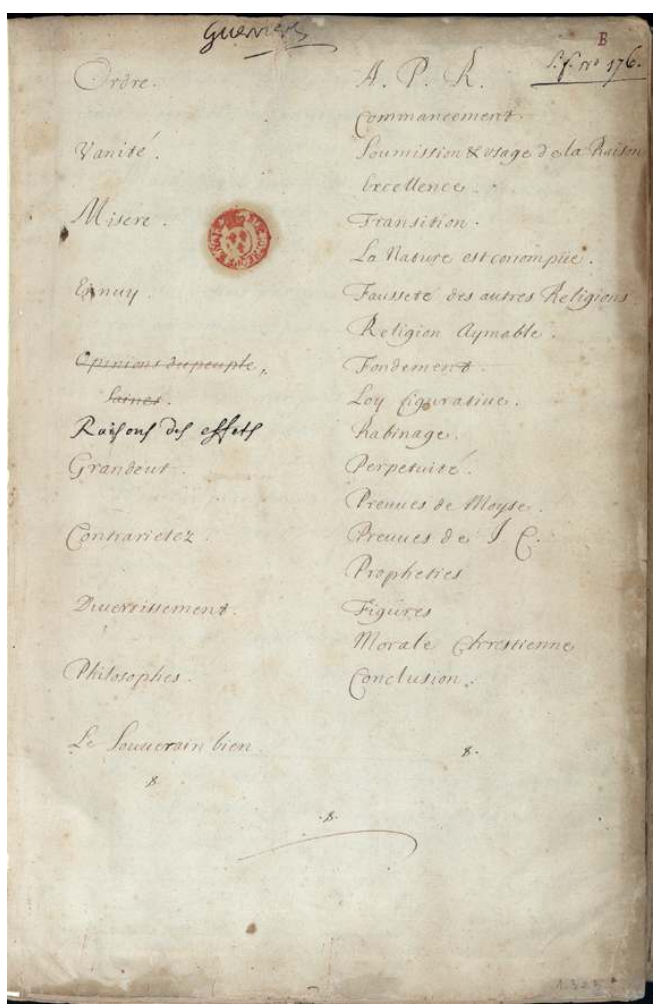

BNF

\section{Principes de transcription communs aux deux Copies}

À l'intérieur de chaque unité, les textes ont été transcrits lisiblement par un même copiste $^{49}$ dans le même ordre, suivant les mêmes règles de présentation :

Les pages sont écrites au recto puis au verso et une réclame est signalée à la fin des pages, sauf lorsqu'il s'agit d'une fin d'unité ${ }^{50}$. Les fins d'unités sont pour la plupart signalées par un ou plusieurs signes distinctifs (./. et $\mathrm{S}$ barré suivi d'un trait final) ${ }^{51}$. Parfois un texte barré ou un renvoi a été ajouté après ces signes ${ }^{52}$.

Une grande marge a été prévue à gauche de chaque page.

Les paragraphes sont aérés, marqués généralement d'un retrait de la première ligne ( alinéa) de taille irrégulière, et assez bien alignés sur la marge de gauche ${ }^{53}$. Les références bibliques sont présentées sans alinéa. Une des consignes était de respecter les paragraphes: si le copiste changeait de paragraphe par erreur il comblait l'espace au moyen d'un trait ${ }^{54}$.

Les changements de papiers ont été, en général, matérialisés par des lignes blanches ou, plus rarement, par des traits de séparation ${ }^{55}$. Mais certains traits pourraient être plus récents. Plusieurs changements de papiers ne sont pas signalés ${ }^{56}$.

La plupart des textes barrés verticalement par Pascal ont été transcrits et barrés verticalement d'un ou plusieurs traits. Les hésitations et les parties de textes corrigées, barrées horizontalement par Pascal, n'ont pas été reproduites à quelques exceptions près. 


\section{Les choix du copiste dans $C_{1}$}

52 L'écriture du copiste est régulière et très lisible, dans une encre brun-violet, identique dans l'ensemble du document. Les nombreuses capitales ou semi-capitales sont souvent difficiles à distinguer des minuscules. Le copiste utilise des graphies anciennes (notamment la graphie monstr - pour le verbe montrer), parfois archaïques (époches pour époques), ainsi que des abréviations classiques pour l'époque composées de caractères surmontés d'un trait (exemples : pnt pour présent, - oe pour - omme, aues pour autres). C'est le travail d'un copiste professionnel.

53 La première page des unités commence toujours sur un nouveau cahier. Si le copiste atteint trop tôt la fin d'un cahier, il réduit la taille de l'écriture et les interlignes, et supprime même les lignes blanches qui devaient matérialiser les changements de papiers ${ }^{58}$. Cela montre que le copiste devait calculer le nombre de feuilles à utiliser avant de commencer un cahier. En revanche, ce processus engendre des pages vierges.

Les paragraphes sont parfois séparés d'une ligne blanche qui correspond soit au trait de séparation de deux idées sur un même papier soit à la volonté du copiste d'aérer le texte. Malheureusement cette présentation se confond avec les lignes blanches qui devaient séparer chaque transcription de fragment et ne permet plus de les détecter de façon certaine. Cette confusion et l'oubli parfois de la matérialisation des changements de papiers nous empêchent d'utiliser cette Copie pour savoir si un papier a été coupé en plusieurs morceaux lors du collage dans le Recueil, et était intact lors de la confection des Copies.

Les lignes sont régulières et bien droites, séparées les unes des autres par un espace régulier, correspondant à ce qu'on pourrait appeler un interligne simple. Les titres ne sont pas soulignés ${ }^{59}$. 
Copie $C_{1 \text { p. }} 81$

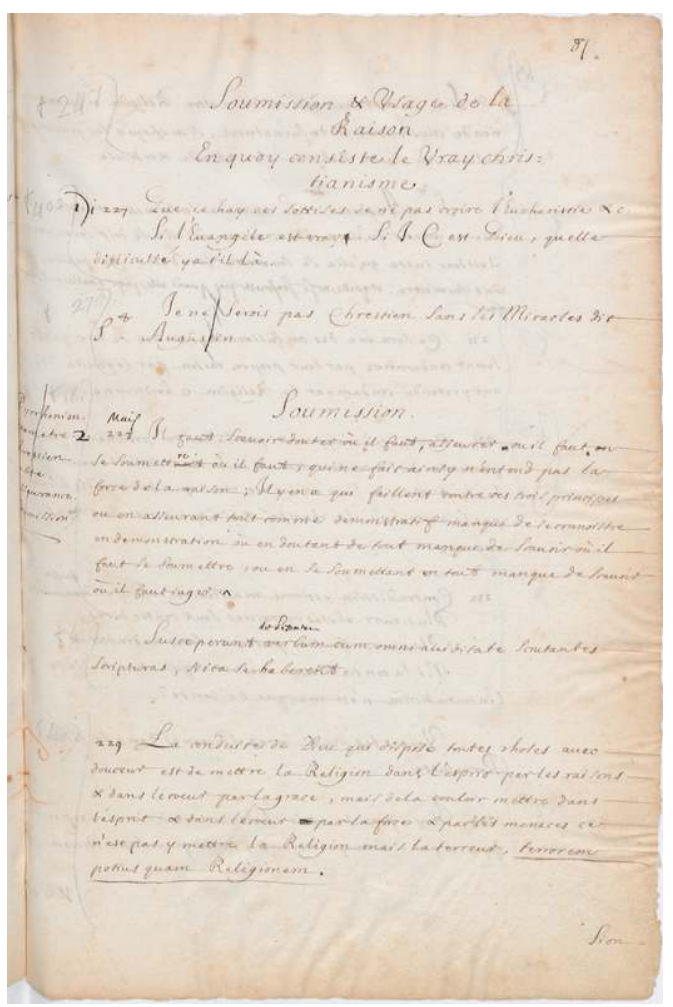

BNF

\section{Les choix du copiste dans $C_{2}$}

L'écriture et l'encre sont les mêmes que dans $C_{1}$. Les caractères sont cependant plus grands. Le copiste utilise toujours des graphies anciennes, mais en modernise d'autres, notamment la graphie - montr - (au lieu de -monstr-) pour le verbe - montrer. Il emploie les mêmes abréviations que dans $C_{1}$ et de nombreuses capitales (plus que dans $C_{1}$ ) ou des semi-capitales toujours difficiles à distinguer des minuscules.

Comme dans $\mathrm{C}_{1}$, l'ajout de lignes blanches entre les paragraphes ne permet pas de savoir de façon sûre où sont les changements de papiers, en particulier lorsque les papiers sont perdus ou lorsqu'il y a un soupçon de découpage post mortem.

Les lignes sont régulières et bien droites, séparées les unes des autres par un espace régulier, plus important que dans $\mathrm{C}_{1}$ (une fois et demi environ) ce qui donne un texte plus aéré. Les titres sont soulignés ${ }^{60}$. La ponctuation proposée par le copiste diffère de celle de $\mathrm{C}_{1}$, et semble plus abondante.

Bien qu'il n'ait pas la contrainte de passer à un nouveau cahier à chaque changement d'unité, il commence en général la transcription d'une unité sur une page impaire (au recto d'un feuillet). Il existe bien sûr des exceptions, notamment lorsqu'il ne change pas de page entre deux unités. Le dossier XXXIV commence au verso du dernier feuillet du dossier XXXIII. Le copiste a bien marqué la fin de l'unité XXXIII par les signes ./. et \$. mais il a continué ensuite comme s'il ne changeait pas d'unité en ajoutant une réclame en bas de la dernière page et en écrivant au verso. Parfois il semble imiter $C_{1}$ (ou peut-être $C_{0}$ ) 
qui laisse deux ou trois pages blanches en fin d'unité : c'est le cas après Philosophes, Fausseté, Loi figurative, Perpétuité, Preuves de J.C., I, titre Miracles, XXXII, XXIII, XXIV, XXV, VII, X, XIII, XV et XVII.

61 Dans le cas de l'unité XXVI p. 347 à 373, le copiste n'utilise que le recto des feuillets et la page 356 ne contient qu'un paragraphe. Nous ne savons pas ce qui a pu provoquer cette présentation.

Le dernier dossier est terminé par trois signes ./.

Copie $\mathrm{C}_{2 \mathrm{p} .} 107$

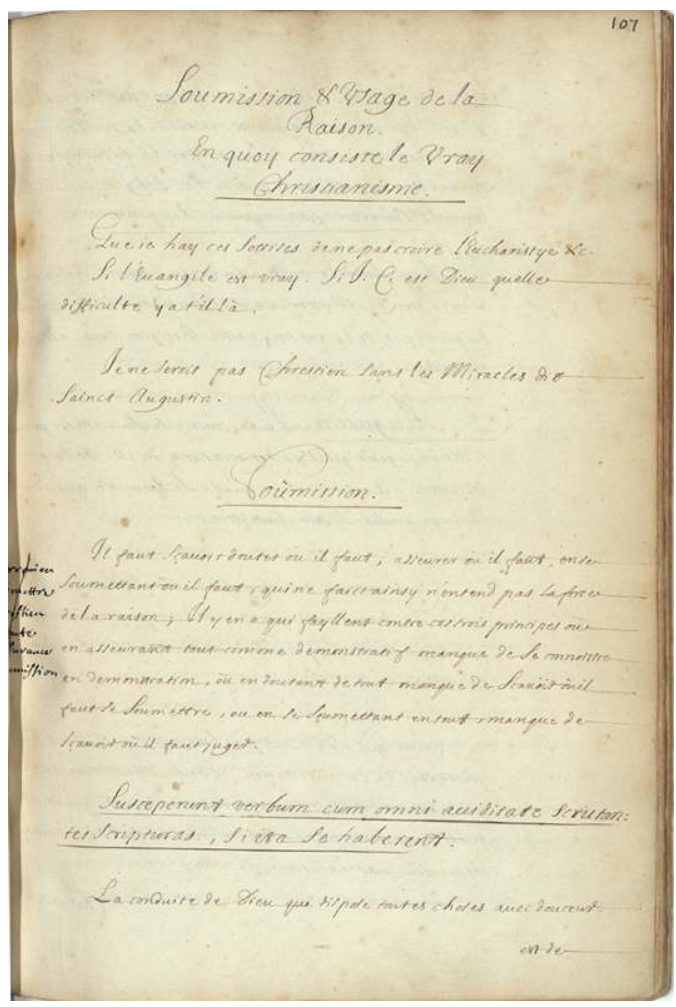

BNF

\section{Fidélité des deux Copies}

\section{Erreurs du copiste}

Les erreurs ont été détectées grâce à la comparaison mot à mot des deux Copies. Une grande partie de ces erreurs a été corrigée par un réviseur. Parmi les erreurs on constate dans chaque Copie une dizaine de sauts du même au même qui n'apparaissent que dans une seule des deux Copies ${ }^{61}$ et plusieurs omissions de petits fragments de phrases ou de quelques mots. La majorité des erreurs sont des fautes de conjugaison et d'accord, l'ajout ou l'omission d'un connecteur ou des confusions dans les mots-outils tels que ces/ses, sa/ la, le/un, etc. ${ }^{62}$

Certains dossiers comptent beaucoup plus d'erreurs dans une des deux Copies mais cellesci sont souvent compensées par des interventions proportionnées du réviseur : 


\begin{tabular}{|c|c|c|c|c|c|c|}
\hline \multirow{2}{*}{ Unité } & \multicolumn{2}{|c|}{$\begin{array}{l}\text { Erreurs du copiste } \\
\text { dans } C_{1} \text { mais corrigées }\end{array}$} & \multicolumn{2}{|c|}{$\begin{array}{l}\text { Erreurs du copiste } \\
\text { dans } C_{2} \text { mais corrigées }\end{array}$} & \multicolumn{2}{|c|}{$\begin{array}{l}\text { Erreurs du } \\
\text { copiste } \\
\text { non corrigées }\end{array}$} \\
\hline & $\begin{array}{ll}\text { par } & \text { le } \\
\text { copiste } & \end{array}$ & $\begin{array}{ll}\text { par } & \text { un } \\
\text { réviseur } & \end{array}$ & $\begin{array}{ll}\text { par } & \text { le } \\
\text { copiste } & \end{array}$ & $\begin{array}{ll}\text { par } & \text { un } \\
\text { réviseur } & \end{array}$ & dans $C_{1}$ & dans $C_{2}$ \\
\hline Divertissement & 2 & 2 & 2 & 3 & 10 & 4 \\
\hline Loi figurative & 3 & 2 & 1 & 11 & 10 & 3 \\
\hline Prophéties & 5 & 12 & 2 & 2 & 1 & 1 \\
\hline II & 18 & 22 & 2 & 1 & 5 & 7 \\
\hline III & 6 & 28 & 9 & 5 & 3 & 3 \\
\hline XXIII & 8 & 25 & 2 & 5 & 11 & 15 \\
\hline XXVI & 3 & 16 & 3 & 2 & 10 & 9 \\
\hline XXXIII & 4 & 26 & 4 & 10 & 4 & 15 \\
\hline$\ldots$ & $\ldots$ & $\ldots$ & $\ldots$ & $\ldots$ & $\ldots$ & $\ldots$ \\
\hline Totaux & 176 & 278 & 98 & 156 & 168 & 180 \\
\hline
\end{tabular}

\section{Fiabilité du copiste}

Dans $C_{1}: 278$ fautes corrigées par un réviseur et 168 non corrigées soit un total de 446 fautes.

dont :

- Liasses titrés : 55 corrigées par un réviseur et 70 non corrigées soit un total de 125 fautes 67 (0,66 fautes par page).

- Autres dossiers : 223 corrigées par un réviseur et 98 non corrigées soit un total de 321 fautes

8 (1,14 fautes par page).

Dans $C_{2}: 156$ fautes corrigées par un réviseur et 180 non corrigées soit un total de 336 fautes.

dont:

- Liasses titrés : 74 corrigées par un réviseur et 72 non corrigées soit un total de 146 fautes

71 (0,77 fautes par page).

- Autres dossiers : 82 corrigées par un réviseur et 108 non corrigées soit un total de 190 fautes (0,68 fautes par page). 

deux fois plus de fautes dans la deuxième partie de $C_{1}$, notamment dans les dossiers II $^{63}$, III, XXIII, XXVI et XXXIII.

\section{réviseur a laissé plus de fautes dans Que la loi était figurative et Divertissement. Globalement,} le réviseur a dû compenser les fautes plus nombreuses du copiste dans $C_{1}$.

$C_{2}$ est-elle plus fiable que $C_{1}$ ? La réponse est non, grâce au réviseur.

\begin{tabular}{|l|l|l|l|}
\hline & $\begin{array}{l}\text { Erreurs non } \\
\text { corrigées } \\
\text { dans } C_{1}\end{array}$ & $\begin{array}{l}\text { Erreurs non } \\
\text { corrigées } \\
\text { dans } C_{2}\end{array}$ & $\begin{array}{l}\text { Différences par rapport à la transcription } \\
\text { actuelle des papiers originaux }\end{array}$ \\
\hline $1^{\text {ère partie }}$ & 70 & 72 & 423 \\
\hline $2^{\text {ème partie }}$ & 98 & 108 & 580 \\
\hline Total & 168 & 180 & 1003 \\
\hline
\end{tabular}

\section{Les Copies sont-elles fidèles au manuscrit de Pascal?}

Les erreurs du copiste non corrigées correspondent au maximum à une faute toutes les trois pages. La réponse est affirmative si on tient compte de la difficulté de lire l'écriture de Pascal et le fait que $85 \%$ des différences de transcription par rapport à la lecture actuelle des papiers originaux proviennent surtout des suffixes (temps, nombre) et de mots-outils (adverbes courants, prépositions, articles, adjectifs possessifs, etc.).

La réponse est négative lorsqu'on examine dans le détail, parmi les $15 \%$ des cas restants, les différences de sens très importantes, les mots ajoutés et les expressions omises (dont 3 cas de sauts du même au même).

Nous proposons ci-dessous quelques cas significatifs qui concernent surtout les textes développés.

\begin{tabular}{|l|l|l|l|l|}
\hline Copies & papier & $\mathbf{C}_{1 \mathrm{p} .}$ & $\mathbf{C}_{2 \mathrm{p} .}$ & $\begin{array}{l}\text { Ed. Sellier n } \\
0\end{array}$ \\
\hline face & force & 10 & 26 & 78 \\
\hline marqués & masqués & 11 & 27 & 78 \\
\hline fâcheuses & fausses & 13 & 30 & 78 \\
\hline poésie & prise & 37 & 55 & 134 \\
\hline immortalité & immatérialité & $39 \mathrm{v}^{\circ}$ & 60 & 147 \\
\hline moins estudié & ouï estimer & $47 \mathrm{v}^{\circ}$ & 68 & 162 \\
\hline
\end{tabular}




\begin{tabular}{|c|c|c|c|c|}
\hline $\begin{array}{l}\text { qu'on fait contre les } \\
\text { pyrrhoniens }\end{array}$ & qu'ont faits les pyrrhoniens & 49 & 70 & 164 \\
\hline simples & superbes & 52 & 73 & 164 \\
\hline la renommée & le remuement & 55 & 77 & 168 \\
\hline pique & pipe & 57 & 80 & 168 \\
\hline occupation & passion & $57 \mathrm{v}^{\circ}$ & 81 & 168 \\
\hline balle & barre & 59 & 82 & 169 \\
\hline impérieuse & injurieuse & $69 \mathrm{v}^{\circ}$ & 97 & 182 \\
\hline pas crier & injurier & 79 & 104 & 194 \\
\hline rien & Dieu & 85 & 112 & 221 \\
\hline J.C. & Dieu & $85 \mathrm{v}^{\circ}$ & 113 & 225 \\
\hline abîme & atome & $91 \mathrm{v}^{\circ}$ & 119 & 230 \\
\hline surpasser & surpayer & $95 \mathrm{v}^{\circ}$ & 124 & 230 \\
\hline nous nous voyons & nous voguons & 97 & 124 & 230 \\
\hline prédire & produire & 135 & 163 & 301 \\
\hline grecs & juifs & 169 & 202 & 369 \\
\hline promis & prompt & 171 & 205 & 374 \\
\hline n'être jamais indépendant & n'être jamais que dépendant & $\begin{array}{ll}177 & \mathrm{~V} \\
0\end{array}$ & 210 & 388 \\
\hline tout cela & l'avènement & 191 & 1 & 4 \\
\hline en tire son mystère & les ait réservés & 191 & 1 & 4 \\
\hline sortes & sectes & 197 & 8 & 29 \\
\hline une puissance extrême & un puissant état & 233 & 447 & 691 \\
\hline foisons & faiseurs & 245 & 461 & 694 \\
\hline injuste & juste & 256 & $\begin{array}{l}471 \mathrm{v} \\
0\end{array}$ & 709 \\
\hline des Mèdes & du midi & 275 & $\begin{array}{l}493 \mathrm{~V} \\
0\end{array}$ & 720 \\
\hline satisfaction & sanctification & 289 & 511 & 735 \\
\hline
\end{tabular}




\begin{tabular}{|c|c|c|c|c|}
\hline moisson & maison & 289 & 511 & 735 \\
\hline salut & sabbat & 295 & $517 \mathrm{v}$ & 735 \\
\hline $\begin{array}{l}\text { qu'ils eurent le } \\
\text { commandement }\end{array}$ & que je m'ordonnai & 297 & $519 v$ & 735 \\
\hline prêtres & ancêtres & 297 & $519 v$ & 735 \\
\hline hommes & livres & $\begin{array}{l}305 \mathrm{~V} \\
0\end{array}$ & $527 \mathrm{v}$ & 738 \\
\hline sentences & semences & 331 & 282 & 458 \\
\hline fille & folle & 351 & $305 \mathrm{v}$ & 495 \\
\hline patience & pitié & 353 & $307 \mathrm{v}$ & 500 \\
\hline souverains & savants & 371 & 329 & 556 \\
\hline prophéties & cinq propositions & 382 & 341 & 596 \\
\hline peine & peur & 382 & $341 \mathrm{v}$ & 598 \\
\hline porter le nom d' & poète et non & 385 & 347 & 613 \\
\hline pensée ci-dessus & pensée de l'autre côté & 392 & 361 & 618 \\
\hline système & bigoterie & $\begin{array}{l}425 \mathrm{~V} \\
0\end{array}$ & 399 & 662 \\
\hline la terre & le trône & 430 & $399 v$ & 668 \\
\hline antéchrist & autre & 441 & 237 & 422 \\
\hline parties & partis & 443 & 240 & 425 \\
\hline exposition & Écriture & 444 & 241 & 426 \\
\hline pour faire et pour donner & il faut pardonner & $\begin{array}{l}445 \mathrm{~V} \\
0\end{array}$ & 243 & 428 \\
\hline sales & salut & $\begin{array}{l}455 \mathrm{v} \\
0\end{array}$ & 255 & 438 \\
\hline le figuratif & la figuration & $\begin{array}{l}457 \mathrm{v} \\
0\end{array}$ & $257 \mathrm{v}$ & 440 \\
\hline avec vos paroles et vos cœurs & $\begin{array}{l}\text { comme un particulier et vous } \\
\text { comme }\end{array}$ & 468 & 267 & 450 \\
\hline selon vos auteurs & etc. Autrement & $\begin{array}{ll}469 \mathrm{~V} \\
0\end{array}$ & 270 & 450 \\
\hline
\end{tabular}




\section{Sauts du même au même communs aux deux Copies et non corrigés}

$\mathrm{C}_{1} \mathrm{n}^{\circ} 74$ p. 305 et $\mathrm{C}_{2 \mathrm{p} .} 527$ (unité XIX) :

2. Que leur discours exprime tres clairement la promesse des biens temporels \& qu'ils disent neanmoins que leurs discours sont obscurs \& que leur sens [saut du même au même] n'estoit pas celuy qu'ils exprimoyent adecouvert \& que par consequent ils entendoyent parler d'autres sacrifices, d'un autre Liberateur \&c.

Il manque ne sera point entendu : d'où il paraît que ce sens secret (saut probable sur sens).

$\mathrm{C}_{1} \mathrm{n}^{\circ} 105$ p. 353 et $\mathrm{C}_{2 \mathrm{p} .} 307 \mathrm{v}^{\circ}$ (unité XXIV) :

Abraham ne prit rien pour luy mais seulement pour ses Serviteurs, ainsy le juste ne prend rien pour soy du monde [saut du même au même] mais seulement pour ses passions desquelles il se sert comme Maistre en disant à l'une va \& vien [...]

Il manque ni des applaudissements du monde (saut sur du monde).

$\mathrm{C}_{1} \mathrm{n}^{\circ} 136$ p. 379 et $\mathrm{C}_{2 \mathrm{p} .} 337 \mathrm{v}^{\circ}$ (unité XXV) :

[...] \& peut estre que cela vient de ce que naturellement [saut du même au même] il ne se peut tromper dans le costé qu'il envisage comme les apprehensions des sens sont toujours vrayes.

Il manque l'homme ne peut tout voir, et de ce que naturellement (saut sur de ce que naturellement).

La présence de ces trois sauts du même au même dans $C_{0}$ montre que cette Copie n'était pas la première version des transcriptions, qu'il y a donc eu une version antérieure $C_{-1}$ qui a aussi disparu.

81 Est-ce que d'autres erreurs ont été faites lors de la première transcription des papiers? C'est probable et elles sont encore à rechercher en étudiant minutieusement chaque papier original. Certaines sont connues telles que la séparation entre les textes Sel. 67 et 68 que Michel Le Guern ( $\left.n^{\circ} 31\right)$ regroupe en un seul fragment pour tenir compte de la reconstitution de Pol Ernst (Album, p. 118-119): le copiste de Pascal, qui utilisait des feuillets séparés, avait changé de feuillet au milieu du fragment.

\section{La table des titres}

Les deux Copies et la deuxième partie de $C_{2}$ commencent par une table de 29 titres présentée sur deux colonnes. Le $R O$ ne contient aucun papier original qui lui corresponde. La première table de $C_{1}$ et la table de $C_{2}$ sont suivies de 27 ensembles de textes classés dans le même ordre que cette table ${ }^{64}$ et commencent par un titre souvent plus long mais qui rappelle celui de la table. Deux des titres ne correspondent donc pas à un ensemble retranscrit. Parmi les titres transcrits dans les 27 unités seuls trois papiers correspondraient dans le RO: APR, Que la loi était figurative et Soumission et usage de la raison, en quoi consiste le vrai christianisme.

Si la présentation est quasi identique dans les deux exemplaires de $C_{1}$ (deux colonnes de même longueur) on constate que les deux colonnes sont de longueurs différentes dans $C_{2}$. Cette différence est essentiellement due à la présentation d'un titre sur deux lignes dans la première colonne (une seule ligne dans $C_{1}$ ) et à la présentation de trois titres sur une seule ligne par titre dans la deuxième colonne (deux lignes dans $C_{1}$ ).

Dans la première table de $C_{1}$, La nature est corrompue est signalé par un trait penché (même encre que le reste de la table) et Prophéties est écrit sans s. 
quelques rares textes barrés ont été transcrits dans les $\operatorname{Copies}^{65}$, c'est le seul titre barré Aucune unité transcrite ne porte ce titre. En revanche, deux fragments (Sel. 128, 129), qui portent ce titre, ont été transcrits dans l'unité Raisons des effets. Cette expression est aussi utilisée dans un fragment dont le titre est Raisons des effets (Sel. 126) : « Il est donc vrai de dire que tout le monde est dans l'illusion, car encore que les opinions du peuple soient saines, elles ne le sont pas dans sa tête. Car il pense que la vérité est où elle n'est pas. La vérité est bien dans leurs opinions, mais non pas au point où ils se figurent. [...] ». Est-ce que le titre Raisons des effets a simplement remplacé Opinions du peuple saines ou est-ce que la liasse Opinions du peuple saines a été regroupée avec la liasse Raisons des effets? On constate que l'écart entre le titre Opinions du peuple saines et Raisons des effets dans la liste est le même qu'avec le titre Ennuy. Si le titre Raisons des effets est un autre titre pour Opinions du peuple saines, ce n'est pas la meilleure façon de les présenter. L'écartement n'aurait pas dû être aussi important et surtout se confondre avec les autres. Deux interprétations sont possibles: soit cette présentation ne reflète plus de façon exacte l'hésitation provoquée par le choix du titre, soit elle correspond à deux étapes du classement, 1/ deux liasses indépendantes Opinions du peuple saines (2 papiers) et Raisons des effets (19 papiers), 2/ Une seule liasse de 21 papiers $^{66}$.

J. Mesnard (ibid., 1971, p.26) fait aussi remarquer que le titre d'unité La nature est corrompue (non barré) correspond aux deux papiers transcrits à la fin de la liasse I (Sel. 35 et 36), et que le titre du texte Sel. 35 a été souligné dans la Copie $C_{1}\left(p .197 \mathrm{v}^{\circ}\right)^{67}$ parce que ce titre était le titre de la liasse et non pas du fragment ${ }^{68}$. Le papier original (RO p. 485) ne porte pas de titre et rien ne permet de dire qu'il en portait. Contrairement au cas précédent la liasse n'aurait donc pas été supprimée mais aurait simplement changé de place.

\section{Que représente cette table?}

88 Est-ce une simple table des matières pour annoncer les unités qui suivent et conserver son ordonnancement ou est-ce la transcription d'un document laissé par Pascal ?

89 En relisant dans la préface d'Étienne Périer, «La premiere chose que l'on fit fut de les faire copier tels qu'ils estoient \& dans la mesme confusion qu'on les avoit trouvez. », on perçoit le désarroi de la famille devant la masse confuse des papiers laissés par Pascal, mais aussi le sentiment que cette confusion n'était qu'apparente et qu'il ne fallait rien perdre. Représenter un tel trésor comportait le risque d'oublier quelque chose, de faire des erreurs et devait faciliter la compréhension de ces écrits. La fidélité relative des Copies qui nous sont parvenues montre toute la difficulté de déchiffrer l'écriture de Pascal et de comprendre le texte. On se doute bien que cela ne s'est pas fait en une seule étape: il y a probablement eu une première copie qui se voulait exacte, mais qui comportait aussi de nombreuses hésitations, puis une deuxième plus lisible puis $C_{1}$ et $C_{2}$, écrites par un copiste professionnel.

A-t-on voulu établir un état aussi précis que possible du classement des papiers tels qu'ils ont été trouvés ou est-ce que Pascal avait laissé un document qui a été recopié ? La 
réponse dépend en grande partie de l'idée qu'on peut avoir de la perte des titres de liasses transcrits en tête de chaque unité.

91 Parmi les papiers des unités titrées 16 textes, qui étaient probablement sur 15 petits papiers, ont été perdus. Ils correspondent aux textes transcrits dans différentes positions dans les Copies : $3^{\mathrm{e}}, 4^{\mathrm{e}}$ et $8^{\mathrm{e}}$ sur 14 dans Grandeur, $1^{\mathrm{er}}$ et $4^{\mathrm{e}}$ sur 14 dans Contrariétés, $1^{\mathrm{er}}$ sur 7 dans Divertissement, $2^{\mathrm{e}}$ sur 8 dans Philosophes, $1^{\mathrm{er}}$ sur 2 dans Le Souverain bien, $12^{\mathrm{e}}$ sur 22 dans Soumission, $6^{\mathrm{e}}$ et $7^{\mathrm{e}}$ sur 8 dans Transition, $1^{\mathrm{er}}, 22^{\mathrm{e}}$ et $24^{\mathrm{e}}$ sur 27 dans Prophéties, et $12^{\mathrm{e}}$ et $16^{\mathrm{e}}$ sur 25 dans Morale chrétienne. Ces papiers proviennent donc de liasses diverses et étaient dans des positions variables. Seuls 4 papiers étaient au-dessus ou au-dessous d'une liasse.

Des titres et des textes ont-ils été supprimés ou occultés durant l'opération de collage dans le Recueil ? Pour certains titres perdus (Sel. 162), (Sel. 531, 532, 534, 554, 558, 561, 563,570 , etc., du dossier XXV) la probabilité d'avoir été découpés pendant cette opération est importante puisque certains des papiers en conservent des traces significatives (Sel. $558,586,603,607)$. À ces cas il faut ajouter celui du texte Sel. 640 redécouvert au verso d'un papier p. 227, du texte Sel. 641 dont la page 226 du Recueil conserve la trace ${ }^{69}$, ainsi que les textes Sel. 639 et 642 qui faisaient partie du même papier mutilé. Il faut aussi ajouter les titres autographes «Preuves de Moïse " (titre de la liasse ?) que Z. Tourneur avait découvert au verso du $3^{\mathrm{e}}$ papier de la liasse (Sel. 324) et « Aveugler, éclaircir » que nous venons de découvrir au verso du papier original (Sel. 268) ${ }^{70}$. Autre exemple, le titre "Commencement », écrit dans les Copies en tête du texte Sel. 196 et supprimé du papier : la reconstitution partielle du feuillet originel par Pol Ernst (Album, p. 138) montre que le texte Sel. 196 ( $R O$ p. 27, $n^{\circ}$ 5) était sous le texte Sel. 114 ( $R O$ p. 469, n 3). Il s'avère que ce dernier papier conserve des traces du mot Commencement et que le trou que laisse le rapprochement de ces deux papiers correspond au titre manquant.

Sel. 196 (RO p. 27, $n^{\circ}$ 5) et Sel. $114\left(R O\right.$ p. $\left.469, n^{\circ} 3\right)$

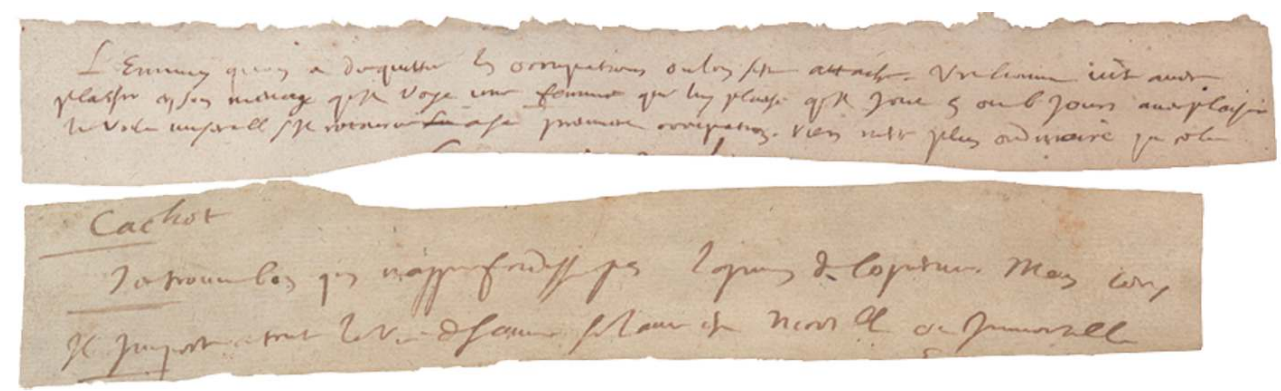

Tous ces cas montrent que ces textes et ces titres existaient lorsqu'ils ont été transcrits la première fois. Faut-il en déduire qu'ils ont été supprimés lors du collage ou qu'ils ont mal été conservés?

Une découverte que nous venons de faire en analysant les fragments de la liasse Misère donne un nouvel éclairage sur les titres de laisses. Le papier RO 21-1 (Sel. 103 : Misère. Job et Salomon) s'avère être l'étiquette de la liasse Misère (nous publierons une étude sur ce point prochainement). Cette étiquette était jusqu'à ce jour passée inaperçue car elle occupe la $18^{\mathrm{e}}$ place de la liasse dans les Copies alors que les deux autres étiquettes connues sont transcrites en tête de leurs liasses respectives (voir l'encadré en fin d'article, p.47). Une des conséquences est de pouvoir envisager qu'un titre de liasse 
corresponde à un des papiers qui porte ce titre dans la liasse quelle que soit sa position, y compris si le titre est suivi d'une pensée de Pascal.

Il nous semble encore plus étonnant que la table des titres, pierre d'angle de l'édifice, ait disparu ${ }^{71}$. Ph. Sellier ${ }^{72}$ rejoint Roger Lacombe (1958) ${ }^{73}$ qui objectait que, si un tel document autographe avait existé, Étienne Périer n'aurait pas écrit dans la préface que les papiers avaient été trouvés sans ordre et sans aucune suite. On ajoutera qu'il est étonnant qu'Etienne Périer ne fasse aucune allusion à cette table.

La table aurait dont été écrite par le premier copiste. Cette hypothèse est consolidée par la différence entre la pauvreté de sa présentation et la complexité du classement, même provisoire, qu'elle est censée représenter ${ }^{74}$.

Toutefois le stockage probablement complexe, si ce n'est chaotique, des liasses ne permet pas d'expliquer la présentation de la table en deux colonnes ${ }^{75}$.

\section{Pourquoi y a-t-il deux tables dans $\mathrm{C}_{1}$ ?} réviseur ${ }^{77}$ et que ces corrections ont permis d'obtenir deux Copies aussi fidèles l'une que
l'autre. Le travail de révision n'est pourtant pas exactement le même dans les deux Copies et il reste à vérifier s'il s'agit bien du même réviseur.

\section{Dans $\mathrm{C}_{1}$}

Le repérage des interventions du réviseur n'est pas simple car de nombreuses interventions ont été faites ensuite par des correcteurs en vue de l'édition et de nombreuses marques ont été ajoutées en marge. Les corrections ont été apportées par le réviseur à la plume (et non au crayon ${ }^{78}$ ou à la sanguine) dans une encre violette un peu plus foncée que celle du copiste. L'écriture est différente de celle du copiste et des 
corrections pour l'édition. Parfois des ajouts sont portés en marge mais ne sont pas suivis d'un trait final contrairement au copiste. Une comparaison mot à mot des deux Copies permet de les distinguer.

L'intervention du réviseur est très efficace. Il oublie cependant de corriger plusieurs fautes d'accord (exemples, p. 12, $45 \mathrm{v}^{\circ}, 59 \mathrm{v}^{\circ}, 69,77$, etc.). Il n'intervient pas sur la ponctuation.

\section{Dans C2}

Contrairement à $\mathrm{C}_{1}$, la Copie $\mathrm{C}_{2}$ contient beaucoup moins d'interventions. Certaines ont été réalisées à la plume dans une encre violet foncé, presque noire. D'autres sont surtout des marques ajoutées en marge au crayon et deux ou trois corrections sont plus récentes. Parmi celles qui ont été faites à la plume, la grande majorité correspond à une correction du texte par un réviseur dont l'écriture a été reconnue comme étant celle d'Étienne Périer.

Le rôle d'Étienne ne s'est pas arrêté à la correction du texte, il a aussi modifié la ponctuation ${ }^{79}$ et souligné les expressions latines qui n'avaient pas été mises en valeur par le copiste.

106 Son intervention est aussi très efficace mais il oublie cependant de corriger de nombreuses fautes d'accords du copiste (exemples p.27, 66, 67, etc.) et s'il corrige le copiste il fait parfois des erreurs p. 175 et 193.

Échantillon de l'écriture du réviseur dans $\mathrm{C}_{1}$

108 (Références : page de $\mathrm{C}_{1}, \mathrm{n}^{\circ}$ Sellier)

$1 v^{\circ}, 43$

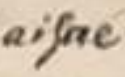

37,133

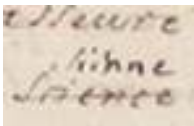

50,164

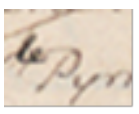

$69 v^{\circ}, 182$

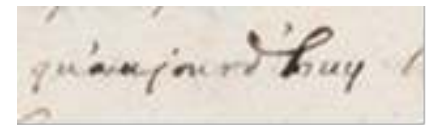


93,230

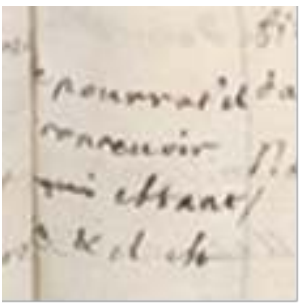

$97 \mathrm{v}^{\circ}, 230$

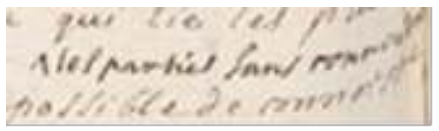

99,230

2,4 in

Simenceste

$119 v, 270$

ipal.

aris pian

$137, v, 304$

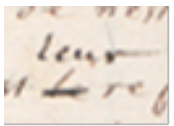

139,305

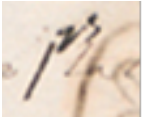

$165 v^{\circ}, 359$

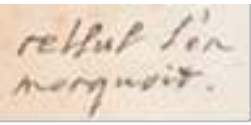

167,361

ige niavers 
167,361

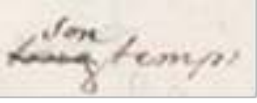

$141 v^{\circ}, 309$

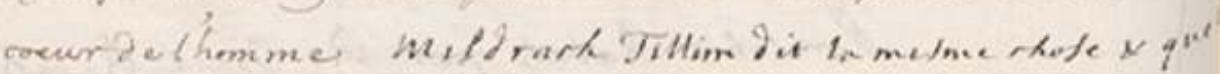

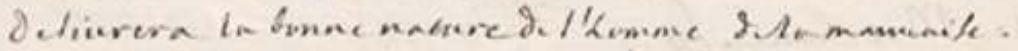

$169 v^{\circ}, 370$

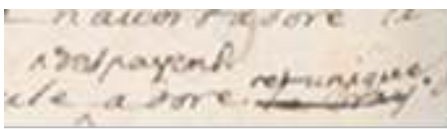

$195 v^{\circ}, 22$

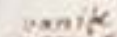

$\operatorname{lon} 12$

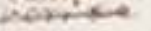

201,680

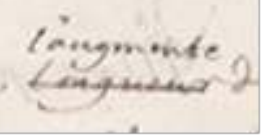

203,680

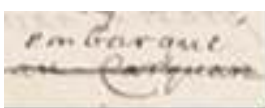

$203 v^{\circ}, 680$

h mavilizary ben infinite' ze ve. infinimeout fecercente a grow

$205 v^{\circ}, 680$

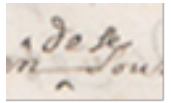

$205 v^{\circ}, 680$

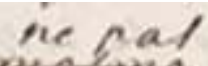

we awa. 
207,680

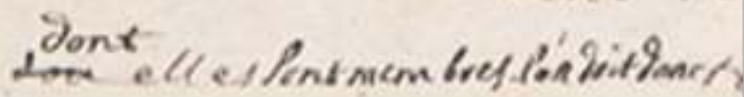

207,680

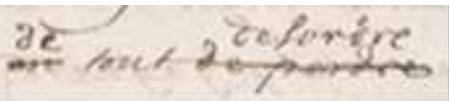

$207 v^{\circ}, 680$

Contive

211,681

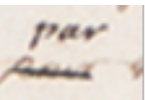

211,681

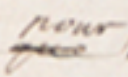

$215 v^{\circ}, 681$

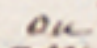

ore

$215 v^{\circ}, 681$

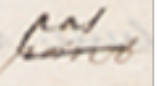

$215 v^{\circ}, 681$

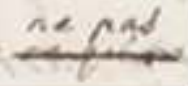

217,681

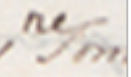

219,682

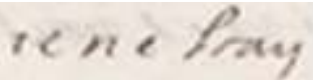

Courrier du Centre international Blaise Pascal, 32 | 2015 
219,682

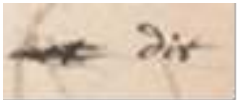

221,684

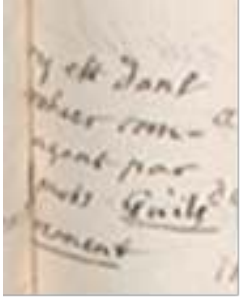

227,690

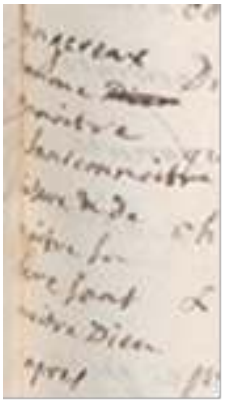

227,690

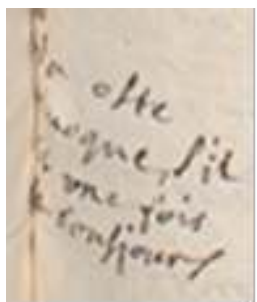

$229 v^{\circ}, 690$

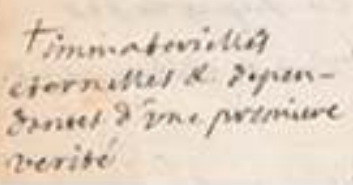

109 Échantillon de l'écriture du réviseur dans C2

110 (Références : page de $C_{1}, n^{\circ}$ Sellier)

Table des titres, 1

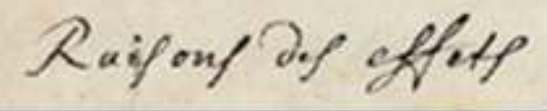

Courrier du Centre international Blaise Pascal, 32 | 2015 
7,22

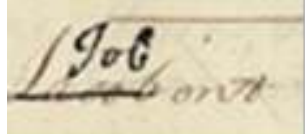

7,23

menis ale.

20,61

at

25,78

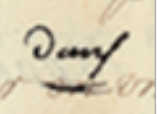

29,78

neture

s. peria

39,102

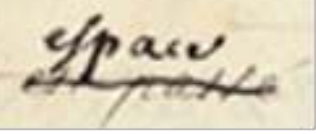

82,169

en

$a^{2 x}$

91,181

Y.e. 
99,182

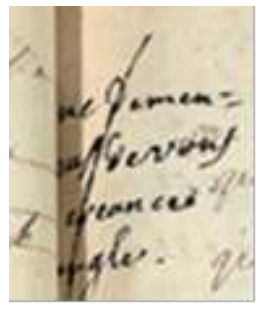

102,182

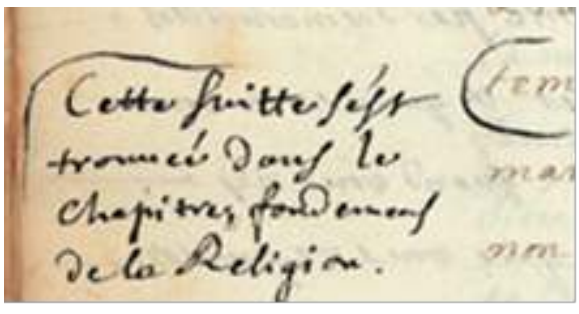

107, 201

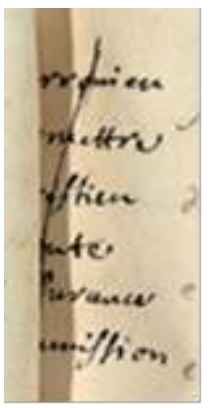

105, 198

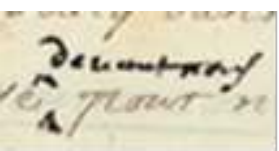

120,230

\section{I.t.}

120,230

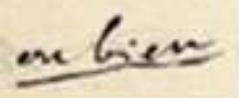




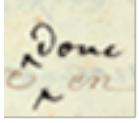

133,242

\section{perir.}

146,269

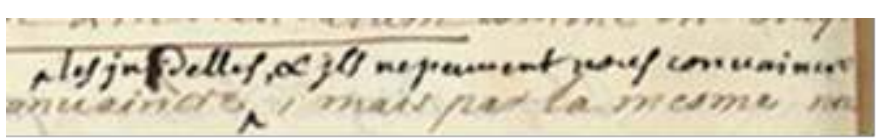

207,381

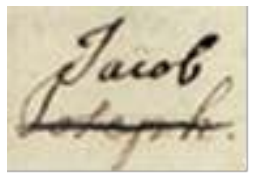

80,168

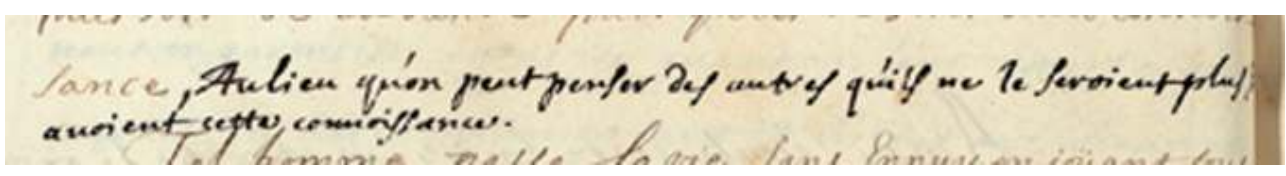

299,486

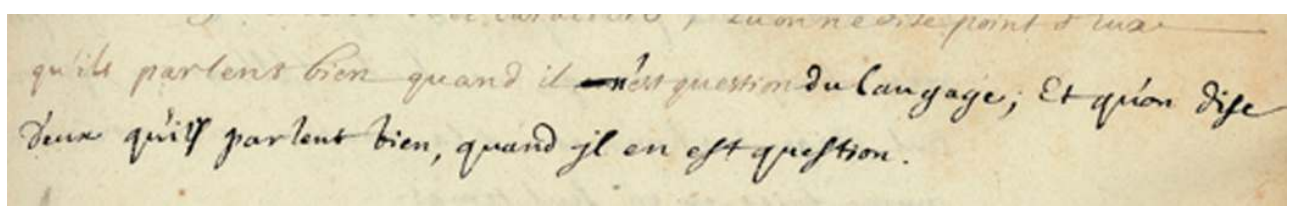

\section{Comparaison des écritures}

Dans $\mathrm{C}_{1}$, les $d$ sont formés avec une boucle fermée en haut; les $s$ finals sont différents de ceux de $\mathrm{C}_{2}$.

Dans $\mathrm{C}_{2}$, la boucle des $d$ n'est pas fermée; les et (notamment, p. 20 et 299) et les $s$ situés à l'intérieur des mots sont caractéristiques et différents de ceux de $C_{1}$. Les $e$ situés en fin de mots se prolongent jusqu'à dépasser la lettre.

113 Les deux écritures sont différentes. Ce n'est donc pas la même personne qui a révisé $C_{1}$ et $\mathrm{C}_{2}$. Il serait intéressant de connaître le nom du réviseur de la Copie $\mathrm{C}_{1}$ et en particulier de savoir si c'est un membre de la famille Périer.

114 Nota: on peut aussi retrouver l'écriture d'Étienne Périer dans $C_{1}$ mais il s'agit de corrections proposées pour l'édition et non pas de simples révisions. 
Par exemple :

C1 p. $221 v^{\circ}$ (Sel. 686)

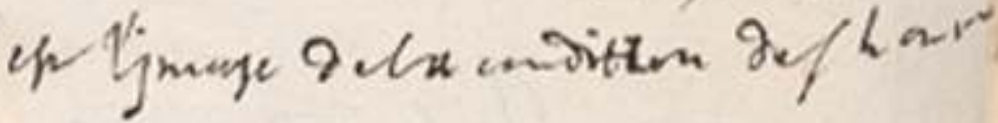

C1 p. $225 v^{\circ}$ (Sel. 690)

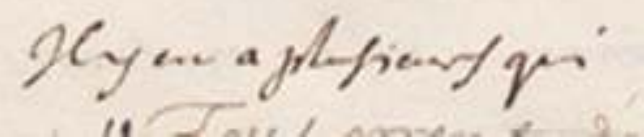

116 Intervention plus récente dans $C_{2}$

117 P. 329, le titre Prou a été transformé en prouinciales. L'écriture est celle, caractéristique, de Pierre Guerrier.

C2 p. 329 (Sel. 558)

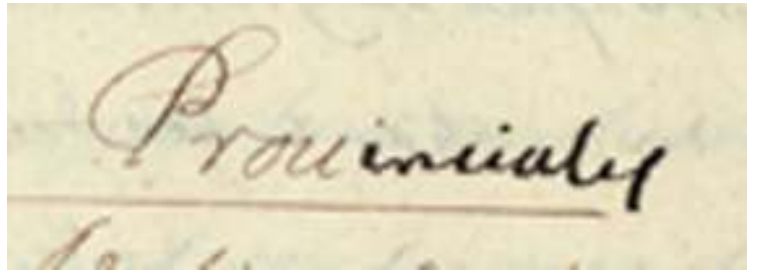

\section{Le cahier Que la loi était figurative p. 539-551 $v^{\circ}$ de $R C_{2}$}

Le Recueil $\mathrm{RC}_{2}$ regroupe différents types de documents, surtout des copies de textes de Pascal, en partie recopiés par Pierre Guerrier. Ce Recueil contient notamment un cahier séparé, constitué de 4 feuilles doubles (16 pages) au filigrane raisin et B. RODIER, similaire à certains cahiers de $\mathrm{C}_{1}^{80}$, et situé à la suite de la Copie $\mathrm{C}_{2}$. Cette copie reproduit maladroitement la liasse Que la loi était figurative.

Le haut de la première page porte une inscription qui semble barrée mais dont le sens nous échappe (ancien numéro de cahier ?). 


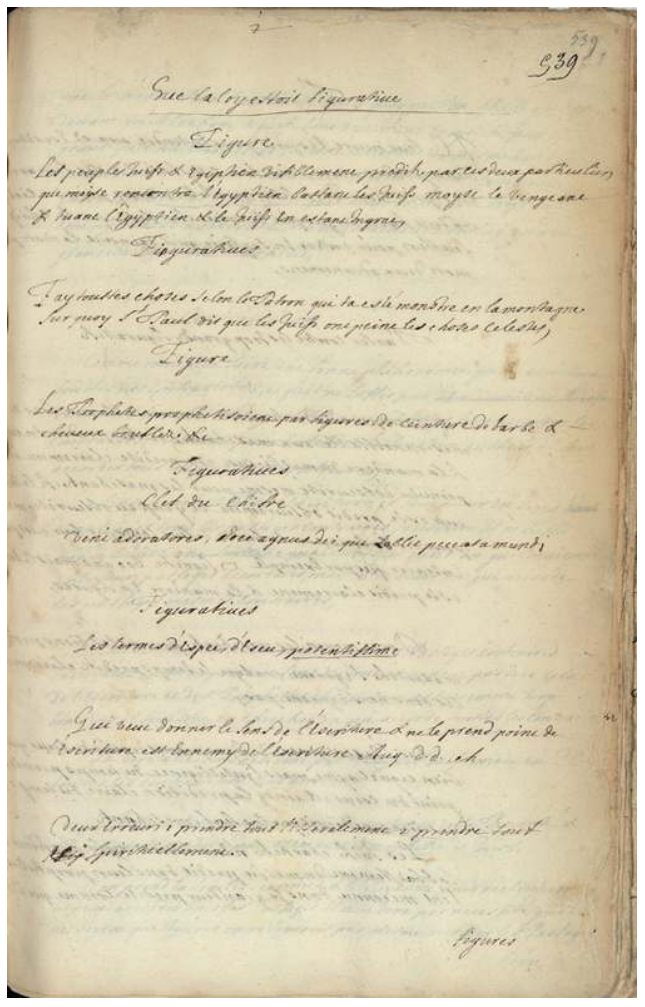

BNF

L'écriture est différente de celle du copiste de $C_{1}$ et de $C_{2}$. Son écriture est régulière mais moins lisible, dans une encre noire identique dans l'ensemble du document ; il utilise des graphies anciennes et de nombreuses capitales ou semi-capitales parfois difficiles à distinguer des minuscules.

La transcription semble confuse et irrégulière :

Les titres sont mal centrés (mais le copiste essaie de corriger cette lacune - voir p. $541 \mathrm{v}^{\circ}$ ), écrits en caractères de même taille que le texte, et seul le titre de la liasse a été souligné. Certains titres, notamment p. 539, ne sont pas séparés du texte qui les précède.

Une marge a été prévue à gauche aussi bien dans les pages impaires (recto) que dans les pages paires (verso). Pourtant il n'utilise pas ces marges : le texte « Les Prophètes ont dit clairement que Israël serait toujours aimé de Dieu et que la loi serait éternelle et ils ont dit que l'on n'entendrait point leur sens et qu'il était voilé. ", qui aurait dû être en marge, a été transcrit dans le corps du texte comme les autres paragraphes.

La plupart des changements de papiers ne sont pas matérialisés par une ou plusieurs lignes blanches ni par un trait de séparation. Le dossier se termine par un signe ./. que l'on retrouve dans les copies $\mathrm{C}_{1}$ et $\mathrm{C}_{2}$.

Les paragraphes sont aérés, souvent marqués d'un retrait de la première ligne de taille irrégulière, et assez bien alignés sur la marge de gauche.

Les lignes sont bien droites, séparées les unes des autres par un espace régulier un peu plus petit que dans $\mathrm{C}_{1}$.

Une réclame a été écrite à la fin de chaque page. 

gloire »; le trait transcrit sans retrait au début de ce paragraphe devait signaler dans la Copie source qu'il fallait faire un retrait; p. 551, le copiste a laissé un espace blanc important entre Isaye et 51 , non matérialisé dans $\mathrm{C}_{1}$ et transcrit Isaye -------- 51 dans $\mathrm{C}_{2}$. Un cas est particulièrement intéressant p. $543 \mathrm{v}^{\circ}$ : le copiste a correctement écrit «le redempteur seroit spirituel et son regne spirituel » alors que et son regne spirituel a été omis dans $C_{1}$ (non corrigée) et $C_{2}$ (ajouté par le réviseur). Ce texte n'est pas plus difficile à lire sur le papier original ( $R O$ p. $\left.15 \mathrm{n}^{\circ} 4\right)$ que la plupart des textes autographes. On peut donc penser qu'il a été correctement transcrit dans la première copie ( $C_{-1}$ ou avant).

$R O$ p. $15 \mathrm{n}^{\circ} 4$

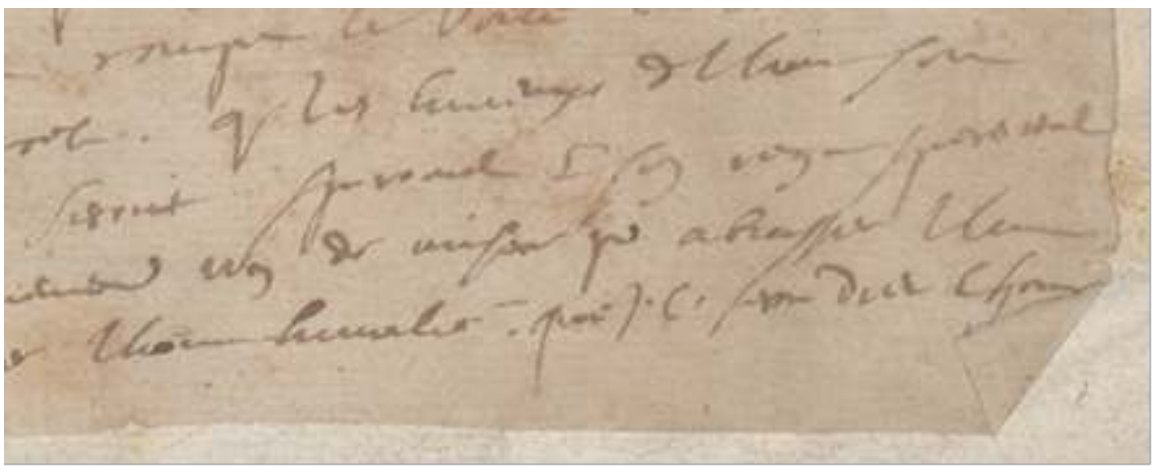

Lomission du copiste dans $\mathrm{C}_{1}$ et $\mathrm{C}_{2}$ pourrait s'apparenter à un petit saut du même au même dans la copie source, c'est-à-dire $C_{0}$, ce qui exclurait que la copie étudiée a utilisé $C_{0}$ comme source. Double erreur du copiste de $\mathrm{C}_{1}$ et $\mathrm{C}_{2}$ ? Ce n'est pas à exclure.

J. Mesnard (Ibid., 1971, p. 15) dit que cette copie est plus proche de $C_{1}$ que de $C_{2}:$ : travail effectué selon la même technique que la copie de $\mathrm{C}_{1}$, donc sous la même direction. Reste, à notre avis, d'un premier déchiffrement, limité probablement à ce chapitre, et qui aura rendu évidente la nécessité de recourir à un copiste plus expérimenté et à l'écriture plus élégante. [...] la copie primitive $\left[\mathrm{C}_{0}\right]$ serait postérieure ».

Notre avis rejoint en partie celui de J. Mesnard : ce n'est pas un cahier de la Copie $\mathrm{C}_{0}$ (ni de $\mathrm{C}_{-1}$ ), car dans cette hypothèse les trous auraient été comblés par un réviseur. Cette copie

Courrier du Centre international Blaise Pascal, 32 | 2015 
n'a pas été réalisée à partir de $C_{1}$ ou de $C_{2}$. Cet essai a été réalisé pour trouver un copiste capable de suivre des principes éditoriaux très stricts similaires à ceux qui ont été suivis dans $C_{1}$ et $C_{2}$. En revanche, les hésitations et les erreurs du copiste sont plus dues à son incompétence qu'à l'état de la copie source, et les copies qui se sont succédé devaient proposer un texte de plus en plus clair. Nous n'avons constaté aucun mot dans cette transcription qui soit absent de $C_{1}$ et $C_{2}$ (donc $C_{0}$ ) et transcrit à partir des textes barrés ou des hésitations de Pascal, ce qui aurait révélé une copie source antérieure. De plus, l'identité de type de papiers entre cette copie et plusieurs cahiers de $C_{1}$ prouve que ces deux copies sont contemporaines.

Cette copie est un test effectué à partir de $\mathrm{C}_{0}$ pour trouver un copiste capable de réaliser soit $C_{1}$ soit $C_{2}$. Dans l'hypothèse de $C_{2}$, rien ne s'oppose à ce que l'essai ait eu lieu après $C_{1}$ si $C_{2}$ lui est postérieur. Aucun élément ne permet à ce niveau de choisir entre ces deux hypothèses.

\section{La Copie $\mathrm{C}_{0}$}

La comparaison minutieuse des deux Copies et de l'essai précédent donnent une idée de l'état des textes dans $C_{0}$ et de sa structure. Il est évident que les textes recopiés de la même façon dans $C_{1}$ et $C_{2}$ reflètent l'état du texte dans $C_{0}$, y compris la mise en paragraphe. Quelques cas, présentés ici, donnent une idée plus précise $\mathrm{de}_{0}$.

\section{Structure physique de $\mathrm{C}_{0}$}

Un commentaire commun à $C_{1}$ et $C_{2}$ fait mention d'un «cecy est dans le cahier commençant par les mots Qu'ils apprennent » ajouté en marge du premier texte de l'unité IV ( $C_{1 \text { p. }} 221$ - ajouté par le réviseur ; $C_{2 \text { p. }} 433$ écrit par le copiste mais sans le faire suivre d'un trait final ce qui aurait pu faire penser à une intervention du réviseur). Dans $C_{1}$, l'unité IV commence sur un nouveau cahier alors que dans $C_{2}$ celle-ci commence sur la même page que la fin de l'unité III et n'en est séparée que par quelques lignes blanches. Nous ne détaillerons pas ce cas ici mais cela montre que l'unité III (qui est concernée par ce commentaire) commençait dans un nouveau cahier dans $\mathrm{C}_{0}$. La notion de cahier associe ici à la fois l'organisation physique et l'unité textuelle.

Deux autres commentaires, présents dans $C_{1}$ et $C_{2}$, font un lien entre deux paragraphes supposés être à 4 pages de distance dans la transcription de la liasse Misère: «\# cy apres page 8 » et «\# cy dessus page 4 » (p. $17 \mathrm{v}^{\circ}$ et 22 dans $\mathrm{C}_{1}$ - p. 37 et 41 dans $\left.\mathrm{C}_{2}\right)$. Il n'y a pas lieu de penser que les pages étaient numérotées dans $C_{0}$. Ces numéros correspondent aux $4^{\mathrm{e}}$ et $8^{\mathrm{e}}$ pages d'un cahier. Il s'avère que cette indication est correcte dans $C_{1}$ mais pas dans $\mathrm{C}_{2}$ : les pages 37 et 41 correspondent aux $7^{\mathrm{e}}$ et $11^{\mathrm{e}}$ pages du cahier 5 et sont distantes de 5 pages au lieu de 4 .

Dans $\mathrm{C}_{2}$, le copiste n'aurait pas dû sauter des pages blanches à la fin de certaines unités, et pourtant une grande partie des unités est suivie de plusieurs pages blanches comme dans $\mathrm{C}_{1}$. Cette anomalie pourrait provenir de $\mathrm{C}_{0}$. C'est notamment le cas après l'unité VII qui n'utilise que les trois quarts d'une page et est suivie de 3 pages blanches.

$C_{0}$ avait donc une structure en cahiers semblable à $C_{1}$ (un cahier par unité). La grosseur de l'écriture du copiste ainsi que la hauteur des interlignes étaient probablement d'une taille intermédiaire entre ceux que l'on peut observer dans $C_{1}$ et $C_{2}$, ce qui fait que certains 
cahiers comportaient parfois un nombre de pages blanches différentes (parfois moins, si ce n'est aucune comme dans les cas où le copiste n'a pas vu les changements d'unités).

\section{Matérialisation des changements de fragments}

Les deux Copies séparent les papiers au moyen d'une ou deux lignes blanches sauf dans quelques cas. La séparation entre les papiers devait être matérialisée par des traits dans $C$ ${ }_{0}$. Le copiste de $C_{1}$ et $C_{2}$ devait avoir pour consigne de les interpréter en laissant des lignes blanches entre chaque papier. $C_{1}$ et $C_{2}$ ont gardé des traces de ces séparations, tout particulièrement dans les dossiers sur les Miracles dans $C_{1}$ et plus souvent dans $C_{2}$, où ces traits ont été recopiés.

\section{Changements d'unités}

Dans les deux Copies les fins d'unités ont été, sauf exceptions, matérialisées par un signe ./. et/ou un ou $3 \mathrm{~S}$ barrés et un trait final, les deux dernières marques étant centrées dans la ligne. De plus, dans trois cas un texte a été écrit dans les deux Copies après ces marques, signe qu'elles étaient déjà présentes dans $\mathrm{C}_{0}$.

\section{Mise en valeur des titres}

Le copiste de $\mathrm{C}_{2}$ a fait suivre chaque titre d'un trait nettement séparé du texte, les a centrés et les a écrits plus gros que le texte. Dans deux cas (p. 34 et 489), le copiste a oublié de souligner le titre, comme dans $\mathrm{C}_{1}$.

Dans $\mathrm{C}_{1}$, les titres sont aussi centrés et écrits plus gros, comme dans $\mathrm{C}_{2}$. Dans deux cas ( $\mathrm{p}$. $141 \mathrm{v}^{\circ}$ et $197 \mathrm{v}^{\circ}$ ), le copiste a souligné le titre comme dans $C_{2}$. Le cas de la page $197 \mathrm{v}^{\circ}$ est particulier car le titre La nature est corrompue pourrait être un titre de liasse.

Dans l'essai de copie Loi figurative on constate que seul le titre de liasse a été souligné.

Il est probable que c'était aussi le cas dans $C_{0}$.

\section{Cas particuliers révélés par l'essai de copie Loi figurative, p. 551}

Le copiste a transcrit «------Et mesme la grace nest que la figure de la gloire ». Le trait transcrit sans retrait au début de ce paragraphe devait signaler dans $C_{0}$ qu'il fallait faire un retrait. Il n'y a pas de cas similaire dans $C_{1}$ et $C_{2}$.

147 Il a aussi laissé un espace blanc important entre Isaye et 51 . Dans $C_{2}$, le copiste a matérialisé cet espace par un trait continu qui devait déjà être dans $\mathrm{C}_{0}$. Dans $\mathrm{C}_{1}$, le copiste a interprété correctement ce trait qui signalait qu'il fallait supprimer cet espace. Le copiste a utilisé le même type de technique dans $C_{1}$ et $C_{2}$ pour supprimer des changements de paragraphe (retours à la ligne).

$\mathrm{C}_{0}$ contenait encore des hésitations de lecture du premier copiste :

a/ Cas de Sel. 565 


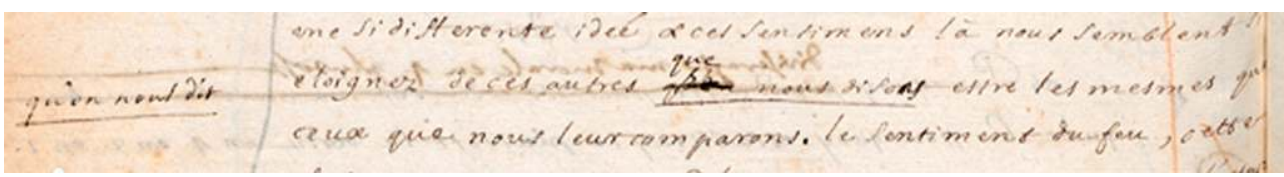

qu'on nous dit : [...] nous en avons co[nceu] une si differente ideé \& ces sentimens là nous semblent si eloignez de ces autres que nous disons estre les mesmes que ceux que nous leur comparons. [...]

Le copiste a d'abord écrit qu'on nous disoit dans le corps du texte puis a corrigé en que nous disons et ajouté qu'on nous dit en marge pour conserver l'autre lecture.

$C_{2}:$ p. $331 v^{\circ}$

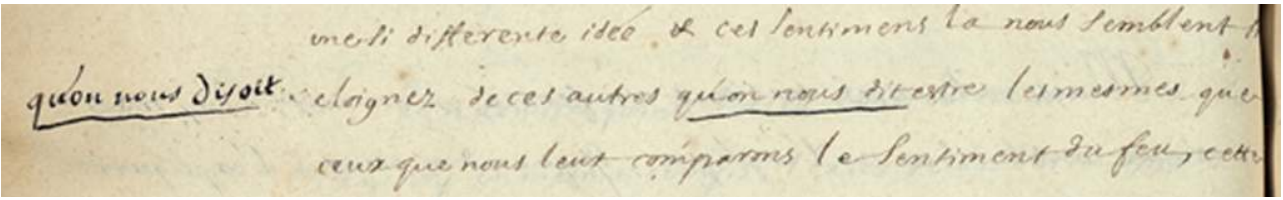

qu'on nous disoit : [...] nous en avons conceu une si differente idée \& ces sentimens la nous semblent si eloignez de ces autres qu'on nous dit estre les mesmes que ceux que nous leur comparons [...]

Cette fois-ci c'est le réviseur qui ajoute l'autre lecture en marge. Les deux Copies interprètent différemment le choix laissé dans $\mathrm{C}_{0}$. Il correspond ici à une hésitation de Pascal.

\section{RO p. $433 \mathrm{n}^{\circ} 1$}

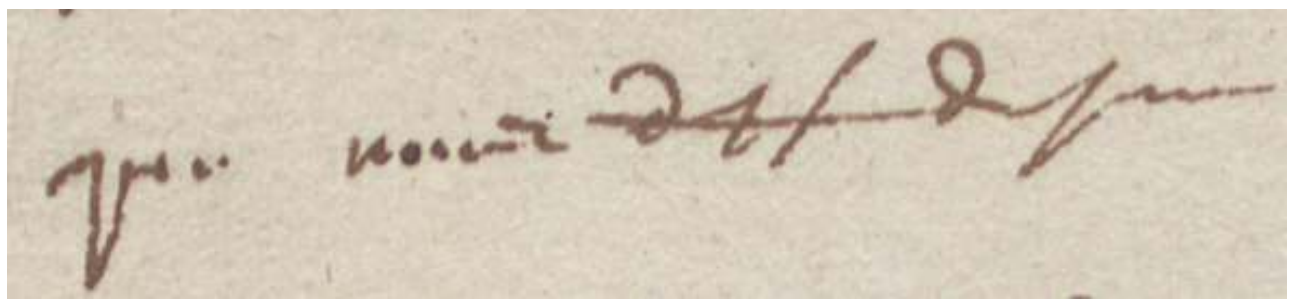

\section{b/ Cas de Sel. 566}

$C_{1}:$ p. $373 v^{\circ}$

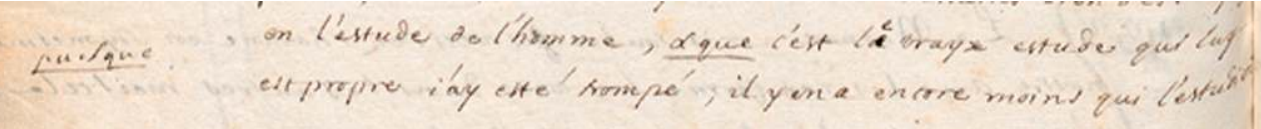

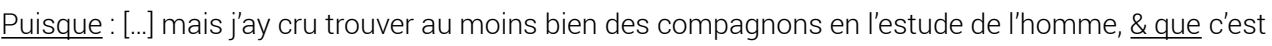
le vray estude qui luy est propre j'ay esté trompé ; il y en a encore moins qui l'estudient 


\section{$C_{2}:$ p. $331 v^{\circ}$ (l'autre lecture est ajoutée par le réviseur)}

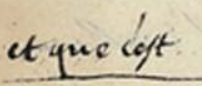

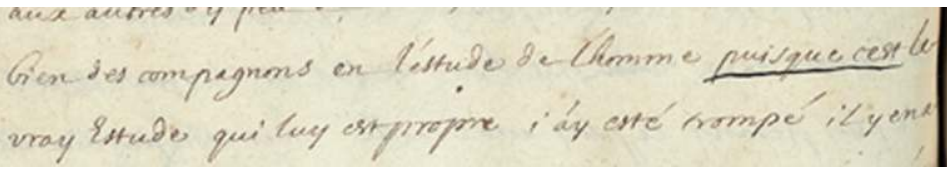

et que c'est : [...] mais j'ay cru trouver au moins bien des compagnons en l'estude de l'homme puisque cest le vray estude qui luy est propre j'ay esté trompé il y en a encore moins qui l'estudient [...]

151 Le premier transcripteur n'a pas dû comprendre le texte et proposer une expression alternative. Pascal n'avait pas hésité.

RO p. $429, \mathrm{n}^{\circ} 3$

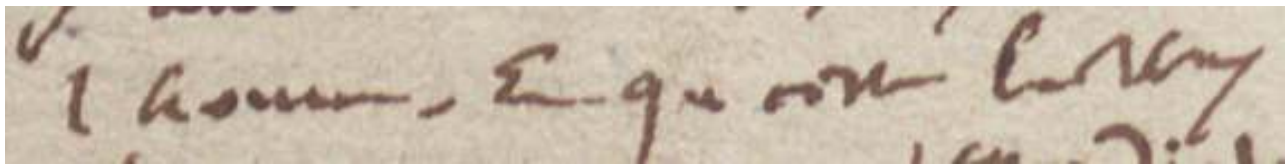

$\mathrm{C}_{0}$ contenait encore des textes barrés, dus à des hésitations de Pascal :

\section{c/ Cas de Sel. 230}

$C_{1}:$ p. $95 v^{\circ}$

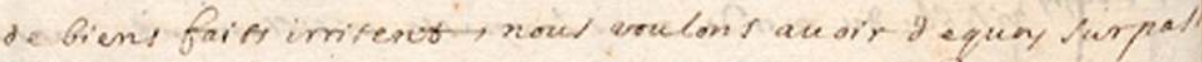

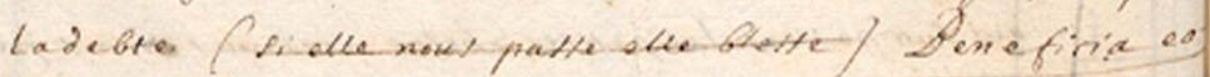

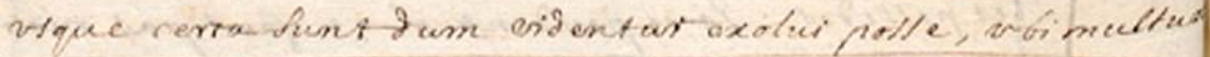

antecerterint proquatia osium resisices

[...] nous voulons avoir dequoy surpasser la debte (si elle nous passe elle blesse) Beneficia eo usque certa sunt dum videntur exolvi posse, ubi multum anteverterint progratia odium redditur.

$\mathrm{C}_{2}:$ p. $123-124$

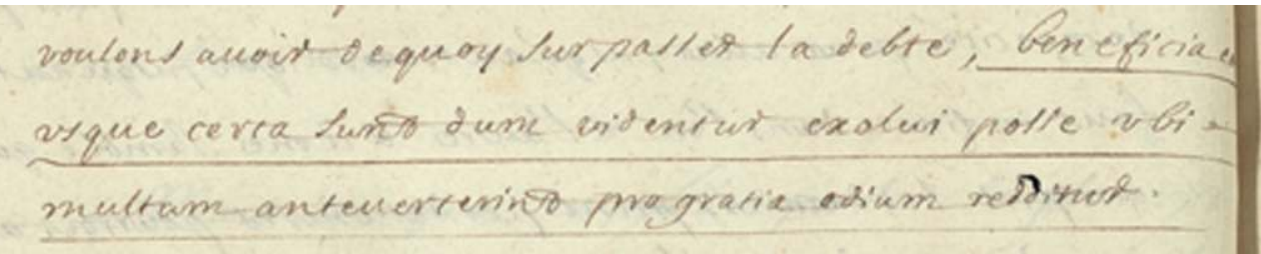

[...] nous voulons avoir dequoy surpasser la debte (si elle nous passe elle blesse) Beneficia eo usque certa sunt dum videntur exolvi posse ubi multum anteverterint progratia odium redditur.

Le texte barré transcrit dans $C_{1}$ mais pas dans $C_{2}$ est un texte barré par Pascal. 


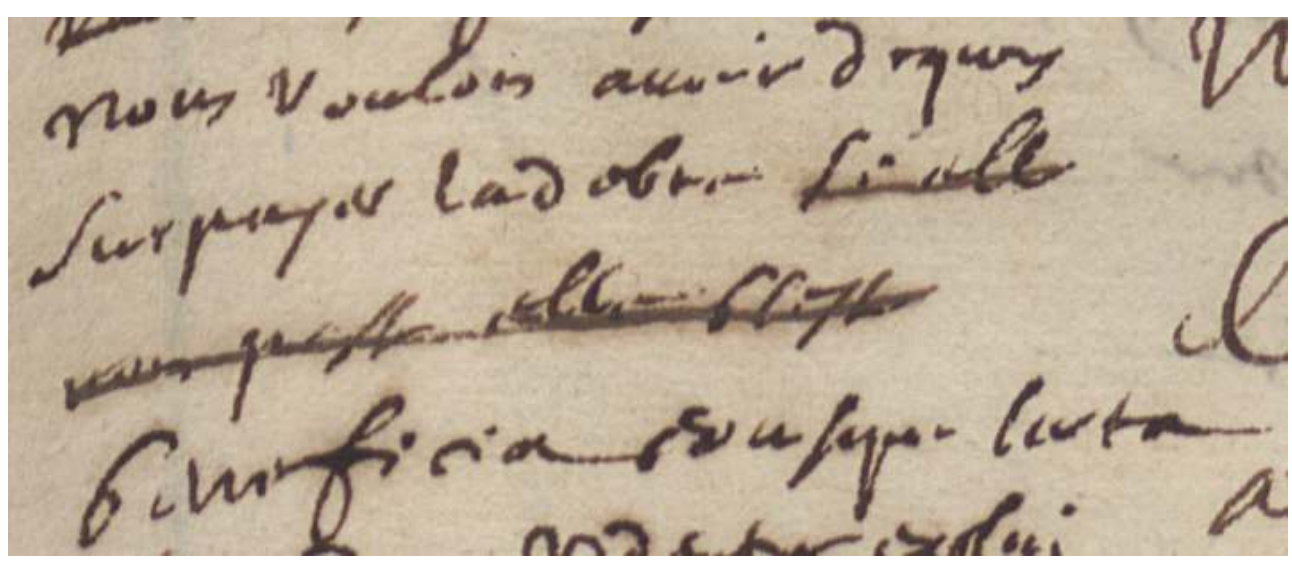

Contrairement à d'autres cas où le texte barré a été conservé dans $C_{1}$ et $C_{2}$ car utiles à la compréhension du texte, celui-ci n'était pas nécessaire et le copiste avait probablement la consigne de ne pas le transcrire.

\section{Mise en valeur de deux textes particuliers}

Le premier texte, intitulé Autre rond, est signalé en marge des deux Copies par le commentaire « Cecy est de la main de M. Constant» $\left(C_{1 \mathrm{p}} .153, C_{2 \mathrm{p}}\right.$. 183). Il est aussi signalé par une sorte de grande accolade gauche qui englobe tout le texte. Ce commentaire n'est pas sur le papier original ( $R O$ p. $491 \mathrm{n}^{\circ}$ 5) mais l'écriture est de Pierre Nicole. Constant est un pseudonyme (le nom de sa mère) utilisé par Nicole uniquement pour un des écrits sur le Formulaire (voir J. Mesnard, Cuvres complètes de Pascal, IV, p. 1319 et Les Pensées ont trois cent ans, p. 23).

Un deuxième texte, Tous les divertissements sont dangereux pour la vie chrétienne..., a été signalé de la même façon par ce type d'accolade gauche dans $C_{1 \text { p. }} 395 \mathrm{v}^{\circ}$ et $C_{2 \text { p. }}$ 369. Le papier correspondant n'est pas dans le Recueil des originaux. Ce texte a paru en 1678 dans les Maximes de Madame de Sablés ${ }^{82}$ Dans $\mathrm{C}_{1}$, le copiste a ajouté un / à la fin du texte et un * dans la marge. 
Copie $C_{2 p .} 369$

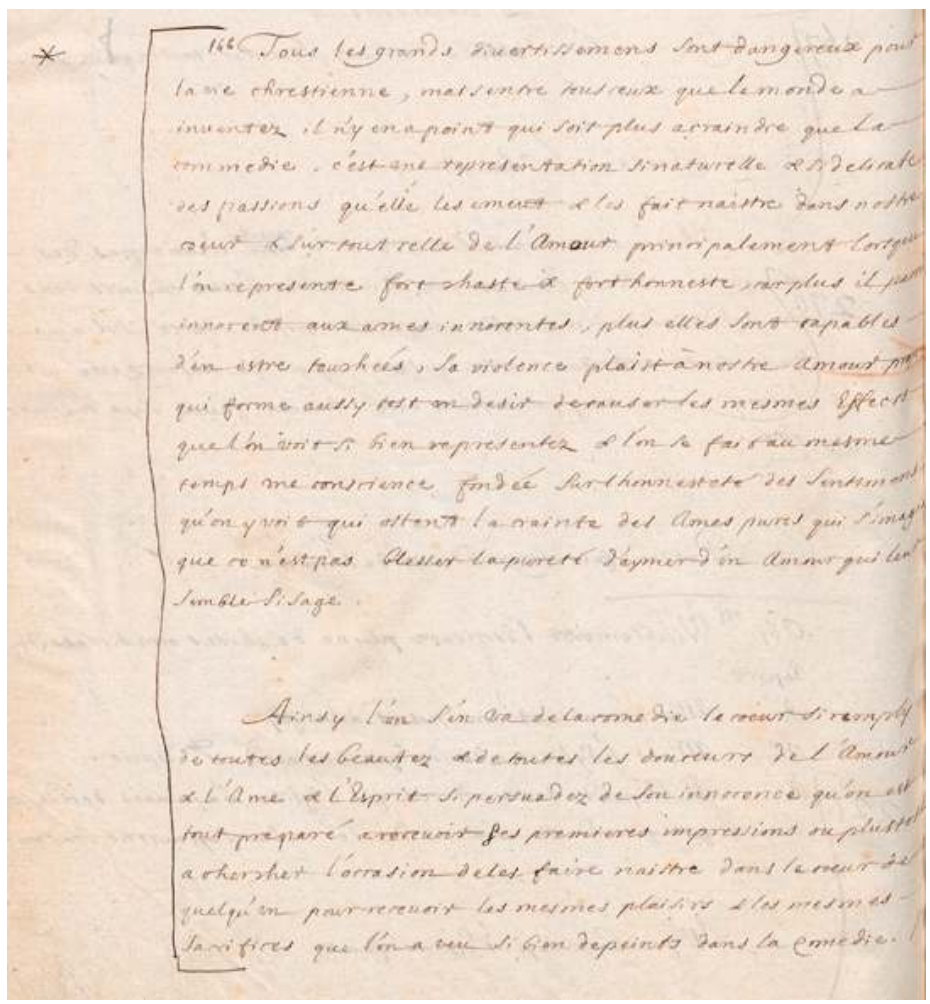

BNF

\section{Chronologie et datation relative des Copies}

Il est utile à ce stade de résumer les informations sur lesquelles se base cette étude : $C_{1}$ et $\mathrm{C}_{2}$ sont issues d'une Copie $\mathrm{C}_{0}$ disparue dont les principes éditoriaux et la structure physique devaient être plus proches de $C_{1}$ que de $C_{2}$; la différence d'ordonnancement entre $C_{1}$ et $C_{2}$ est due à l'entassement des grands dossiers dans $C_{0}$ durant la première de ces transcriptions; $C_{2}$ a été révisée par Étienne Périer; $C_{1}$ a été révisée par un autre réviseur ; $C_{0}$ contient des sauts du même au même qui montrent qu'elle pourrait être issue d'une autre Copie disparue $C_{-1}$; l'essai de copie Loi figurative est issu de $C_{0}$ et a été réalisé immédiatement avant ou après $C_{1} ; C_{1}$ a été utilisée comme document de travail pour l'édition princeps mais aussi pour l'édition revue et augmentée de 1678 .

Il reste à déterminer $\mathrm{si}_{2}$ a été réalisée avant ou après $\mathrm{C}_{1}$ ou en parallèle avec $\mathrm{C}_{1}$.

\section{Est-ce que les différences constatées entre $C_{1}$ et $C_{2}$ permettent de déterminer laquelle des deux a été réalisée en premier?}

La Copie $\mathrm{C}_{2}$ contient un grand dossier de plus que dans $\mathrm{C}_{1}$. Ce dossier, probablement une liasse de 4 papiers, est noté XXXV par L. Lafuma (Laf. 953, 949 et 968) et intitulé Contre la fable d'Esdras par Ph. Sellier (Sel. 415, 416, 417 et 418). L'absence de ce dossier dans $C_{1}$ peut s'expliquer de deux façons : soit le cahier correspondant était à la fin de $C_{1}$ et a été égaré, soit la transcription a été ajoutée à $C_{0}$ après que $C_{1}$ ait été terminée mais avant de 
commencer la transcription des dossiers sans titre dans $C_{2}$. Cette dernière hypothèse supposerait que $C_{2}$ a été réalisée après $C_{1}$. On notera que ce texte n'est pas dans l'édition de Port-Royal.

Le titre du grand dossier Miracles a été ajouté par une tierce personne ${ }^{83}$ dans une page blanche de $C_{1}$ (encre plus noire et en très gros caractères) alors qu'il a été écrit par le copiste dans $\mathrm{C}_{2}$. Le titre du grand dossier Preuves de la religion par le peuple juif, les prophéties et quelques discours a été écrit par le copiste dans $C_{2}$ mais est absent de $C_{1}$. Aucun papier du Recueil des originaux ne porte ces titres ${ }^{84}$.

L'instruction mettre cela au chap. ${ }^{\text {re }}$ commençant par A.P.R., absente de $C_{1 \text { p. }} 121$, a été ajoutée en marge de $\mathrm{C}_{2 \text { p. }} 147$ et en face d'un texte intitulé APR pour demain. L'édition Lafuma 1951 intègre ce texte dans Laf. 149. L'édition Sellier 2000 le publie deux fois dans Sel. 185 et 274. L'écriture semble être celle du copiste et l'instruction est suivie d'un trait final caractéristique du copiste. Le papier original (RO p. $\left.57 \mathrm{n}^{\circ} 3\right)$ correspondait au début de la deuxième feuille double du texte intitulé $\mathrm{APR}^{85}$ et à la fin de ce texte. Il aurait été trouvé dans la liasse Fondements. C'est ce qu'indique le commentaire donné en marge dans la Copie $C_{1 \text { p. }} 75$ (écriture du copiste) et dans $C_{2 \text { p. }} 102$ (ajouté par le réviseur) : Cette suitte s'est trouvée dans le chap. Fondemens de la Religion ${ }^{86}$.

Copie $\mathrm{C}_{2 \text { p. }} 147$

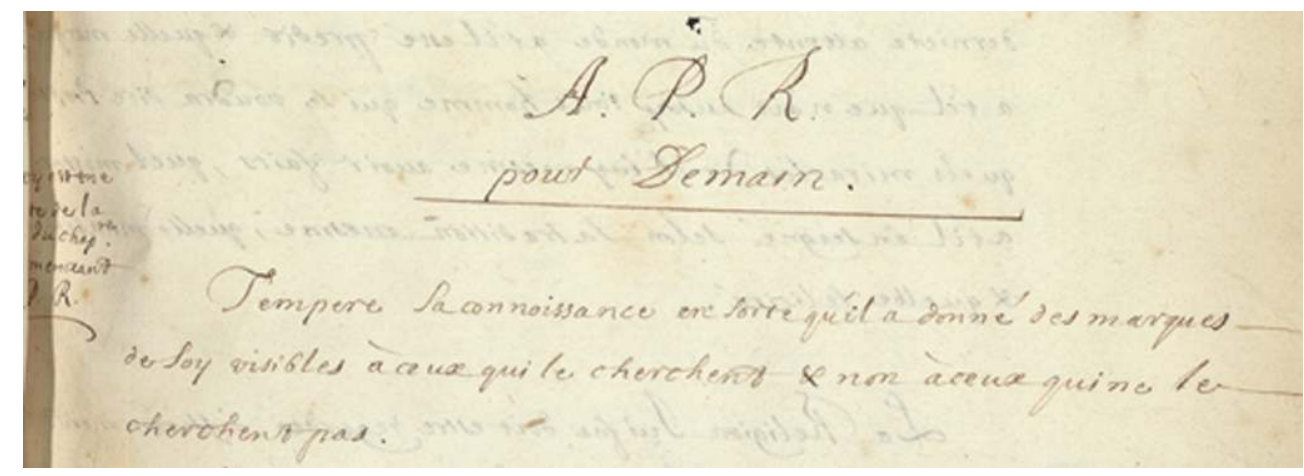

L'omission d'une expression par le copiste dans une des deux Copies, et corrigée - ou non - par un réviseur, peut s'interpréter de quatre façons ${ }^{87}$ :

1. C'est une erreur du copiste et le réviseur l'a corrigée.

2. C'est une erreur du copiste et le réviseur a omis de la corriger.

3. L'expression était absente de $C_{0}$ et n'a été ajoutée dans $C_{0}$ qu'après la confection de la première des deux Copies, sans que la Copie dans laquelle elle manque ne soit corrigée par le réviseur.

4. L'expression était absente de $C_{0}$ et a été ajoutée par le réviseur à la fois dans $C_{0}$ et la Copie où elle manquait.

Les hypothèses 1 et 2 nous empêchent d'en déduire la chronologie des Copies. En revanche, on peut déduire des hypothèses 3 et 4 que la partie fautive de la Copie concernée a été réalisée avant la partie non fautive de l'autre Copie.

Le cas présent $\left(C_{2 p}\right.$. 102) correspond soit à l'hypothèse $\mathrm{n}^{\circ} 1$ soit à la $\mathrm{n}^{\circ} 4$ appliquée à $\mathrm{C}_{2}$. On constate aussi que le cas de $C_{1 \text { p. }} 121$ correspond soit à l'hypothèse $n^{\circ} 2$ soit à la $n^{\circ} 3$ appliquée à $C_{1}$. L'une des deux omissions correspond donc nécessairement à une erreur du copiste et non à l'absence de l'information dans $C_{0}$. L'efficacité reconnue du réviseur 
de $C_{1}$ pourrait faire supposer que l'information présente dans $C_{2 p}$. 147 était absente de $C_{0}$, ce qui correspondrait à l'hypothèse $n^{\circ} 3$ appliquée à $C_{1}\left(\right.$ donc $C_{1}$ avant $\left.C_{2}\right)$. Mais l'argument est faible.

L'analyse des différences entre $C_{1}$ et $C_{2}$, qui sépare le travail du copiste et des réviseurs et tient compte de l'édition de Port-Royal et des papiers originaux, ne permet pas de déterminer de façon certaine laquelle a été réalisée en premier.

\section{Un cas significatif ? Sel. 465 (texte non retenu dans l'édition de Port-Royal)}

RO p. $110 n^{\circ} 2$ :

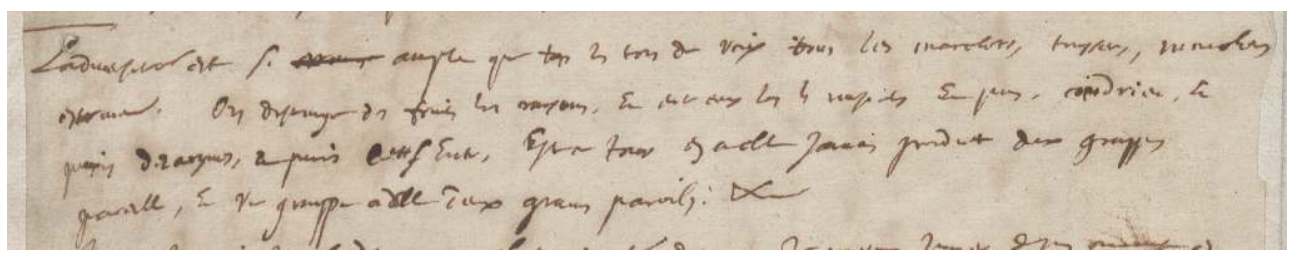

La diuersité est si ? ? ? ample que tous les tons de voix tous les marchers, toussers, mouchers, esternuers. On distingue des fruits les raisins ${ }^{88}$ Et entre ceux la, les muscats Et puis, coindrieu, Et puis desargues ${ }^{89}$, Et puis Cett $s$ Ente, Est ce tout en a elle jamais produit deux grappes pareille, Et Vne grappe a Elle deux grains pareils. \&c

L'expression sont différents est absente du fragment original et rend la phrase illisible.

$C_{2 p .} 287$

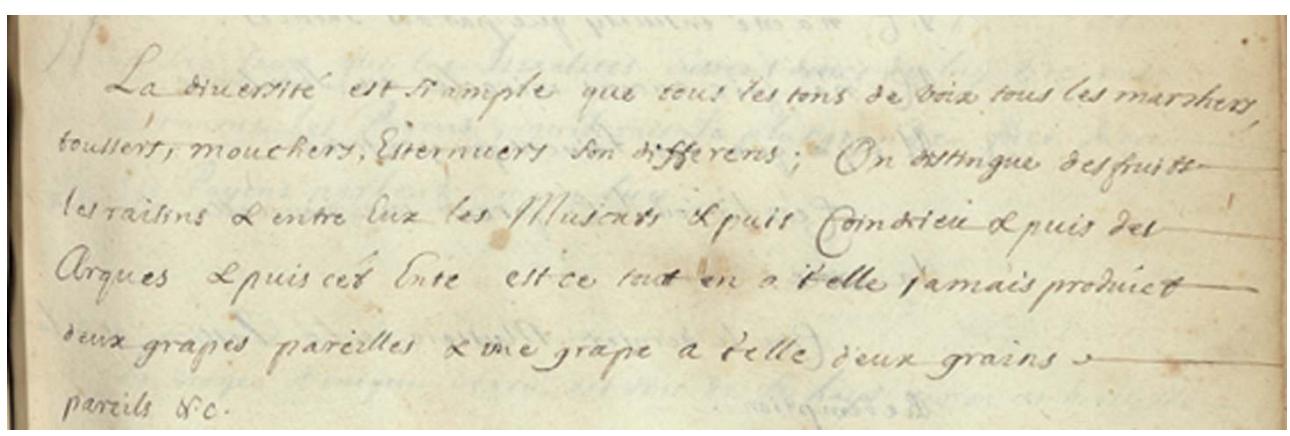

La diversité est si ample que tous les tons de voix tous les marchers,

toussers, mouchers, Esternuers SON DIFFERENS ; On distingue des fruits les raisins \& entre Eux les Muscats \& puis Coindrieu \& puis DES

ARQUES \& puis CÉT Ente est ce tout en a t'elle jamais produict

deux grapes pareilles \& une grape a t'elle deux grains pareils \&c.

Le texte n'a pas été corrigé par le réviseur. Son[t] differens est inclus dans le texte par le copiste (avec une coquille - à moins qu'il ait compris sons différents...). Il écrit bien raisins et non raisons. Il ajoute un s à pareil. Il écrit cet et non cette. Il écrit des Arques comme dans $\mathrm{C}_{1}$. 


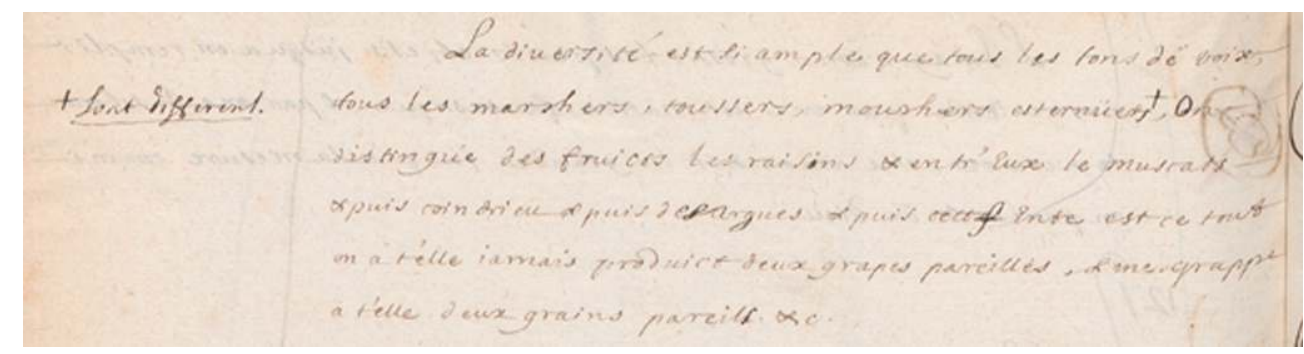

'sont DIFFERENS. : La diversité est si ample que tous les tons de voix, tous les marchers, toussers, mouchers esternüers+, on distingue des fruicts les raisıns \& entr'Eux le muscats \& puis coindrieu \& puis desAraues \& puis cetter Ente est ce tout en a t'elle jamais produict deux grapes pareilles, \& une grappe a t'elle deux grains pareils. \&c.

Le réviseur a ajouté sont differens. en le signalant dans le corps du texte par un renvoi. La révision va semble-t-il au-delà d'un simple rapport avec une copie, notamment ce surprenant $f$ mis à la place du $e$ de cette. Il corrige raisons en raisins. En revanche, il laisse entr'eux au lieu de entre ceux la et corrige desArgues en desArques. Il est difficile de lire ce que le copiste avait écrit sous le $O$ de $O n$, le $O$ étant écrit par le réviseur. Il est probable que le copiste avait écrit on et que le réviseur signalait que On commençait une nouvelle phrase, la phrase précédente se terminant par sont differens suivi d'un point final.

Première hypothèse : en comparant $C_{1}$ à $C_{0}$, le réviseur s'est aperçu que la première phrase n'avait pas de verbe dans $C_{1}$. Cet élément devait aussi manquer dans $C_{0}$ et l'a amené à faire une (mauvaise) relecture du papier original : notamment cett $f$ au lieu de cette s (le s étant barré). Ces corrections ont ensuite été prises en compte dans $C_{2}$ (sauf cett f) sans que le réviseur intervienne. Ce serait alors un témoin de l'ordre $C_{1}$ puis $C_{2}$.

Deuxième hypothèse : la copie $C_{0}$ conservait encore en marge les traces des hésitations de la lecture de l'original. Le copiste aura omis de les interpréter dans $\mathrm{C}_{1}$, ce qui a provoqué une intervention du réviseur. Dans ce cas on ne peut pas en déduire la chronologie de $C_{1}$ / $\mathrm{C}_{2}$.

\section{Réalisation simultanée des deux Copies?}

Le processus d'entassement des grands dossiers dans $\mathrm{C}_{0}$ entre les deux Copies exclut que les deux Copies aient été réalisées en même temps. En revanche, il n'exclut pas les hypothèses de réalisation de $C_{1}$ et $C_{2}$ par parties : par exemple $1^{\text {ère }}$ partie de $C_{1}$ puis $1^{\text {ère }}$ partie de $C_{2}$ puis $2^{\text {ème }}$ partie de $C_{2}$ puis $2^{\text {ème }}$ partie de $C_{1}$.

\section{Réalisation par parties des deux Copies?}

Le fait que le copiste ait réalisé les deux Copies et qu'il soit un professionnel a ici toute son importance car un copiste professionnel est plus sensible à l'évolution du choix de certaines graphies que l'honnête homme. On sait notamment que les protes ont dû s'adapter progressivement aux règles que les imprimeurs leur imposaient, notamment dans les graphies à utiliser, et celles-ci ont évolué au gré des dictionnaires. La comparaison de la deuxième édition des Pensées avec la troisième édition de janvier 1671 est caractéristique. Le texte de 1671 n'a pas été recomposép ${ }^{90}$. Seules quelques corrections 
(parfois aberrantes) ont été faites sur la composition de janvier 1670 (première édition), elle-même modifiée (deuxième édition). Parmi les corrections on peut aussi constater que certaines graphies ont été modernisées ; c'est en particulier le cas du verbe -montrer et de ses dérivés verbaux (écrits -monstrer ou -montrer) : deuxième édition : 39 -monstr- et 9 montr-; éd. 1671: 16 -monstr- et 32 -montr-. Cette modernisation sera encore plus nette dans l'édition de $1678^{91}$.

On peut constater le même changement chez notre copiste.

Dans $C_{1}$ :

- 81 -monstr- et 1 -montr- dont

- Liasses titrés : 40 -monstr- et 0 -montr-

- Autres dossiers : 41 -monstr- et 1 -montr-

Dans $\mathrm{C}_{2}$ :

- 8 -monstr- et 72 -montr- dont

- Liasses titrés : 5 -monstr- et 36 -montr-

- Autres dossiers (dont XXXV mais hors copie Guerrier) : 3 -monstr- et 36 -montr-

Nota : seule la graphie -monstr- est utilisée dans l'essai Loi figurative (8 occurrences).

La règle suivie par le copiste exclut que les Copies aient été réalisées par parties. De plus, cette règle nous semble un indice supplémentaire - si ce n'est décisif - qui suggère que $C_{1}$ a été réalisée avant $C_{2}$ et qu'une période plus ou moins longue s'est déroulée avant de réaliser $\mathrm{C}_{2}$, ce qui expliquerait pourquoi le copiste n'a pas remarqué le déclassement des grands dossiers.

\section{Conclusions}

De nombreux indices (structure, filigranes, graphies) suggèrent que $C_{1}$ a été réalisée entièrement avant $C_{2}$. De plus, n'est-il pas logique de penser que le copiste a réalisé sa première Copie en conservant la même structure que le document qu'il avait sous les yeux, d'autant que cette structure était adaptée à une étude des textes par plusieurs personnes? Cette structure a cependant l'inconvénient de gâcher beaucoup de papier et montre qu'il est difficile de calculer de façon sûre le nombre de feuilles nécessaires par unité. Le choix de la structure de $C_{2}$ correspond à un choix économique tout en aérant un peu plus le texte, choix que le copiste a eu du mal à respecter.

La variété des filigranes des cahiers de $C_{1}$ n'est pas surprenante et correspond probablement à deux ou trois marchands papetiers qui fournissaient un même négociant. L'unicité du filigrane des quelque 150 feuilles utilisées dans $C_{2}$ l'est beaucoup plus et pourrait correspondre à une commande spéciale. Une étude approfondie de ce filigrane serait intéressante mais il y a peu de chance qu'elle nous donne une date précise.

S'agit-il d'une Copie témoin pour la famille ? C'est très probable mais cette dernière n'avait pas prévu l'incident de l'empilage des grands dossiers. On peut aussi se demander pourquoi les dossiers de $C_{1}$ n'ont pas été reclassés comme dans $C_{2}$, et si le dossier XXXV manquait dans $C_{1}$, pourquoi c'est toujours $C_{1}$ qui a été utilisée pour ajouter des fragments dans l'édition de 1678.

177 La chronologie des Copies $C_{1}$ et $C_{2}$ est essentielle pour déterminer dans quel ordre Pascal avait laissé ses dossiers, mais la conclusion reste fragile, car elle suppose que les Copies antérieures n'ont pas bouleversé ce classement. 


\section{Datation relative}

Les précédentes études permettent d'envisager la chronologie suivante :

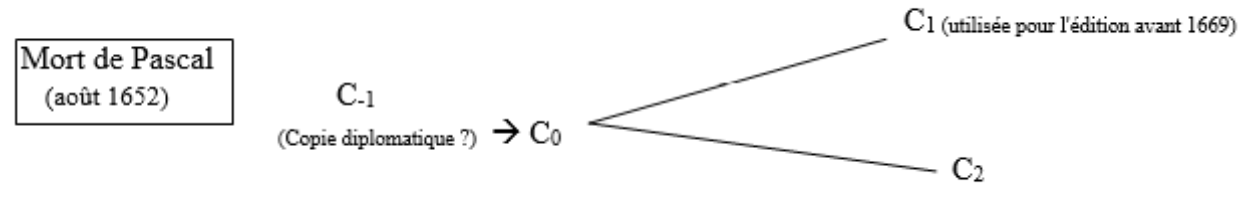

La préface d'Étienne Périer parle du travail de copie puis des hésitations qui ont abouti à l'édition en janvier 1670 :

[...] l'on eut un très grand soin après sa mort de recueillir tous les écrits qu'il avoit faits sur cette matiere. [...] Et tout cela estoit si imparfait \& si mal écrit qu'on a eu toutes les peines du monde à le déchiffrer. La premiere chose que l'on fit fut de les faire copier tels qu'ils estoient \& dans la mesme confusion qu'on les avoit trouvez. Mais lors qu'on les vit en cet estat, \& qu'on eut plus de facilité de les lire \& de les examiner que dans les originaux, ils parurent d'abord si informes, si peu suivis, \& la pluspart si peu expliquez, qu'on fut fort longtemps sans penser du tout à les faire imprimer, [...] Mais comme il y avoit plusieurs manieres de l'executer, l'on a esté quelque temps à se déterminer sur celle que l'on devoit prendre. La premiere qui vint dans l'esprit \& celle qui estoit sans doute la plus facile, estoit de les faire imprimer tout de suite dans le mesme estat qu'on les avoit trouvez. [...]

mais ne permet pas de se faire une idée précise du nombre de Copies intermédiaires et du temps nécessaire à leur réalisation puis à l'étude des copies par le Comité éditorial.

L. Lafuma (Ibid., Introduction p. 9) estime qu'il a fallu plus d'un an de travail pour réaliser la première Copie dont parle Étienne Périer, ce qui nous paraît peu s'il s'agit d'une copie diplomatique. Il propose pour son exécution la période 1662-1663, en se fondant sur le fait qu'un commentaire inscrit en marge d'un texte, transcrit dans les deux Copies $\left(\mathrm{C}_{1 \mathrm{p} .}\right.$. $153, \mathrm{C}$ 2 p. 183), est de la main de M. Constant, pseudonyme de Pierre Nicole en 1662. Cette date correspond probablement à la première transcription du papier correspondant, ce qui fait en effet commencer les travaux de déchiffrement quelques mois au plus après la mort de Pascal.

Le privilège accordé par le roi à Florin Périer ${ }^{92}$ pour publier divers documents écrits par Pascal a été signé le 27 décembre 1666 : « Notre ami et feal conseillier en notre Cour des aydes de Clermont Ferrand, le sieur Périer, nous a faict remontrer qu'il auroit cy devant obtenu nos lettres de permission pour faire imprimer des Traittez de l'équilibre des liqueurs [...] et que depuis l'édition desdits traittez on auroit imprimé à son insceu plusieurs Fragments de mathématiques [...] et appréhendant qu'on ne fist imprimer aussy les Fragments et Pensées qu'il a du mesme autheur sur diverses matieres, et qu'on les donnast informes au publicq, il nous auroit très humblement supplié de luy vouloir accorder nos lettres de permission [...] durant l'espace de cinq ans, à compter du jour qu'il sera achevé d'imprimer [...]».

L. Lafuma estime aussi que le Comité éditorial a commencé à travailler en 1664 ou 1665 et que le Privilège a été demandé lorsque le travail du Comité a permis de choisir le type d'édition envisagé. Il est possible que le Comité pouvait en 1665 consulter la seule Copie lisible $\mathrm{C}_{0}$. 
181 J. Mesnard (Ibid., 1971) ${ }^{93}$ rappelle que la Copie $C_{1}$ conserve les traces manuscrites de propositions de corrections d'Arnauld et Nicole dont on a tenu compte dans l'édition et que leurs interventions ne concernent que quelques cahiers. Ce sont les seules traces manuscrites de membres du Comité. On sait aussi qu'Arnauld et Nicole sont entrés dans la clandestinité et y demeurent jusqu'à la paix de l'Église (1668). Il est fort probable que, ne pouvant pas se déplacer sans risque et travailler directement avec le Comité, ces cahiers leur ont été transmis pour qu'ils y notent leurs commentaires.

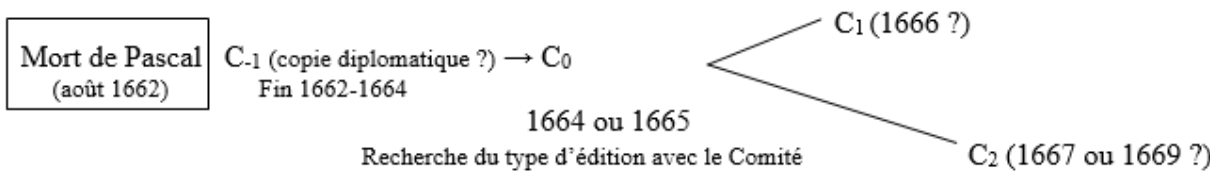

Il est plus difficile d'avancer une date pour la réalisation de $C_{2}$. Le seul élément dont on dispose sont des lettres qui attestent l'implication active de Gilberte Périer dans le projet d'édition. Gilberte était à Clermont-Ferrand et Étienne, qui la représentait auprès du Comité, était à Paris. Il fallait donc que Gilberte ait en main une Copie suffisamment claire pour vérifier le travail du Comité qui a dû commencer en 1666-67 après l'obtention du Privilège et la réalisation de $C_{1}$. A-t-elle fait réaliser $C_{2}$ dans ce but ou utilisait-elle $C_{0}$ ? Aucune information ne permet de trancher.

\section{Deuxième partie : utilisation des Copies}

\section{La Copie $\mathrm{C}_{1}$ : une copie de travail pour l'édition}

Ph. Sellier (Ibid., 2010, Introduction p. 31) résume ainsi l'impression donnée par la Copie C ${ }_{1}:$ « le texte de $\mathrm{C}_{1}$ a été abîmé par des corrections de mains multiples (Arnauld, Nicole, É. Périer), à tel point qu'on ne discerne plus toujours ce qui est copie originelle et ajouts [...] ». De plus, de nombreuses marques tracées à la plume, à la sanguine, ou à la mine de plomb, témoignent d'un travail intense dont les phases et leur chronologie sont difficiles à cerner.

L'encre et l'écriture régulière du copiste facilitent le repérage de ses interventions (hésitations, corrections). Celles du réviseur sont moins évidentes mais ont été confirmées par une comparaison minutieuse des deux Copies. Trois autres mains ont été reconnues, d'abord par P. Faugère (1844) puis par J. Mesnard (1971), comme étant celles d'Antoine Arnauld, Pierre Nicole et Étienne Périer qui ont proposé des corrections dans une encre violette plus ou moins foncée puis à la sanguine. Les corrections qui consistent en un simple trait tracé à la plume pour signaler qu'il ne faut pas retenir un fragment, un paragraphe ou une expression, ne sont pas identifiables. Aucune de ces propositions n'a été intégrée au texte de la Copie $\mathrm{C}_{2}$. En revanche, certaines de ces corrections ont été prises en compte dans le texte de l'édition ${ }^{94}$.

\section{Exemples d'écritures}


Arnauld ( $C_{1} n^{\circ} 249$ p. 91 - Transition)

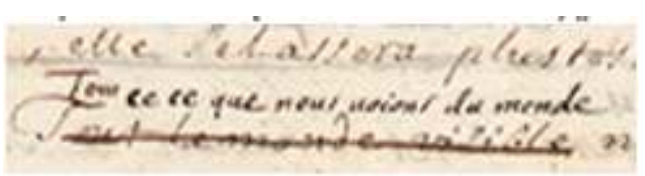

Arnauld $\left(C_{1} n^{\circ} 166\right.$ p. $45 v^{\circ}$, Contrariétés)

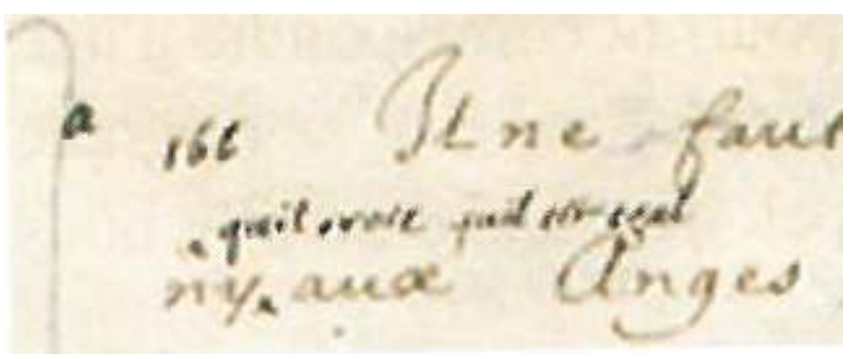

Nicole (C1 n 234 p. 83 - Soumission)

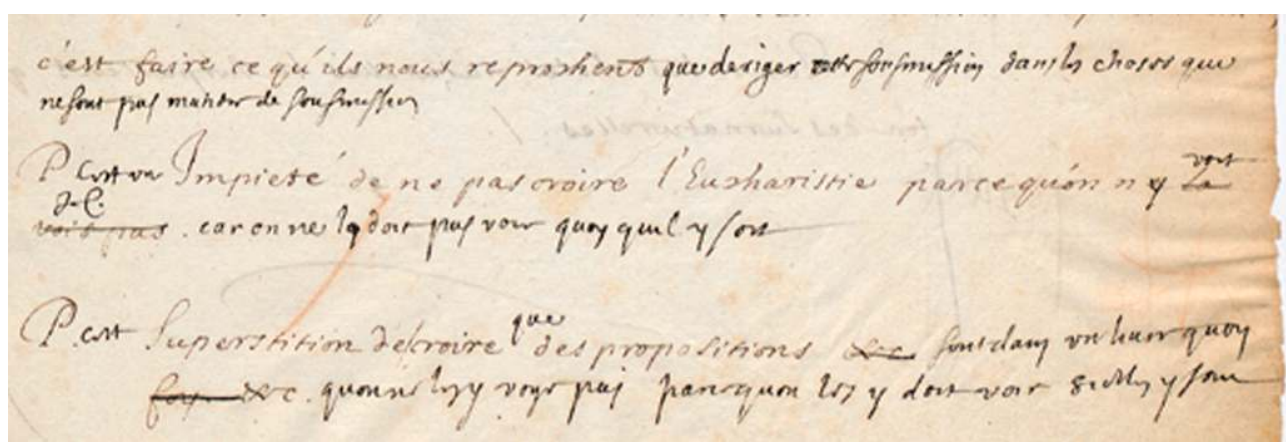

Étienne $\left(\mathrm{C} 1 \mathrm{n}^{\circ} 317\right.$ p. $139 \mathrm{v}^{\circ}$, Loi figurative)

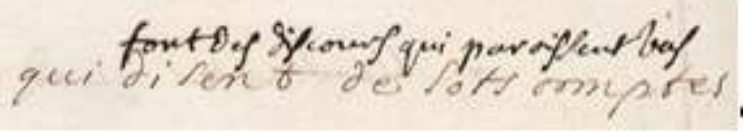

Étienne (C1 n 361 p. 177, Morale chrétienne)

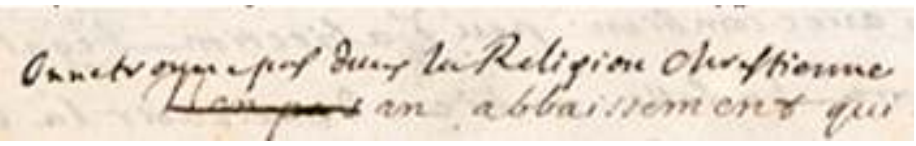

1/ Corrections proposées pour l'édition de 1670

(voir le détail en Annexe)

Si Étienne intervient dans la préparation des éditions de 1670 et 1678, Arnauld et Nicole ne semblent pas avoir laissé de traces liées à l'édition de 1678. Unités concernées par les corrections :

Antoine Arnauld : Grandeur (1) ${ }^{95}$, Contrariétés (2), Transition (2), Fausseté des autres religions (2), Fondements (1), II (1). 
Pierre Nicole : Vanité (5), Souverain bien (1), Soumission (8), Prophéties (4), XVI (1).

Étienne Périer: Philosophes (1), Loi figurative (1), Morale chrétienne (5), XIX (1), XXIII (3), XXVI (3) XXVIII (1), XXX (1) et peut-être III, IV et V dans lesquelles les corrections n'ont été retenues qu'en 1678.

C1 p. 65 (corrections d'Arnauld)

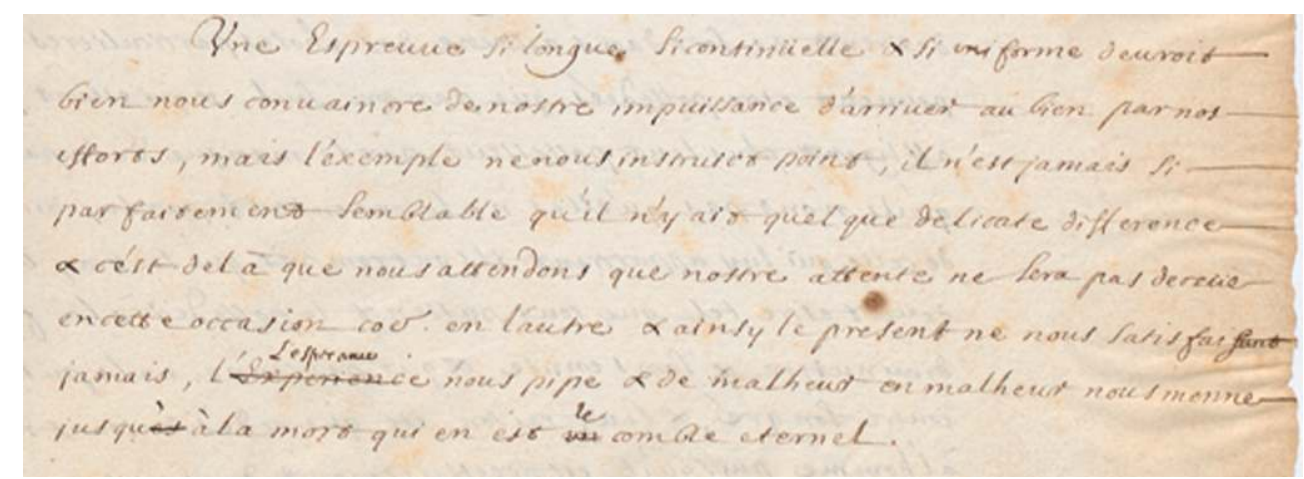

Edition p. 165

Une épreuve fi longue, fi continuelle, \& fi uniforme devroir bien nous convaincre de l'impuifance où nous fommes, d'arriver au bien par nos efforts. Mais l'exemple ne nours inftruit point. Il n'eft jamais fi parfaitement femblable, qu'il n'y ait quelque delicate difference; \& cieft delà que nous attendons que noftre efperance ne fera pas deceüe en cette oc. cafion comme en l'autre. Ainfi le prefent ne nous fatisfaifant jamais, l'ef. perance nous pipe, \& de malheur en malheur nous mene jufqu'à la more

(en violet : les mots corrigés)

En fait, si l'on compare ces traces avec les corrections et les ajouts effectués dans l'édition, on constate qu'elles ne représentent qu'une infime goutte d'eau dans le travail réalisé par le Comité ${ }^{96}$.

191 À part quelques exceptions qui seraient à confirmer, les corrections proposées par Arnauld et Nicole ont été portées sur des cahiers qui concernent des liasses à titres. Les interventions d'Étienne sont plus variées et portent surtout sur les autres dossiers mais 
peu de ses corrections ont été prises en compte dans l'édition de 1670. En revanche, d'autres corrections concernent celle de 1678 et sont probablement associées aux marques diverses ajoutées en marge à la plume et à la sanguine.

\section{2/ Nouveaux fragments proposés pour l'édition de 1678}

Les traces qui ont été laissées pour préparer l'édition de 1678 sont plus importantes en nombre et plus variées. La concordance avec l'édition permet de mettre en évidence deux phases principales de travail: le repérage de quelques fragments déjà retenus dans l'édition de 1670 et une phase complexe de sélection de nouveaux fragments pour 1678 .

\section{Phase de repérage et corrections}

193 Selon J. Mesnard (Ibid., 1971, p. 19), les corrections proposées à la sanguine seraient dues à Étienne ${ }^{97}$. C'est aussi lui qui utilise la sanguine pour repérer certains textes qui ont déjà été intégrés dans l'édition de 1670 et en barrer. Ces deux opérations ont pu être simultanées. Cette phase s'accompagne de marques diverses ajoutées en marge à la sanguine.

Marques ajoutées en marge à la sanguine.
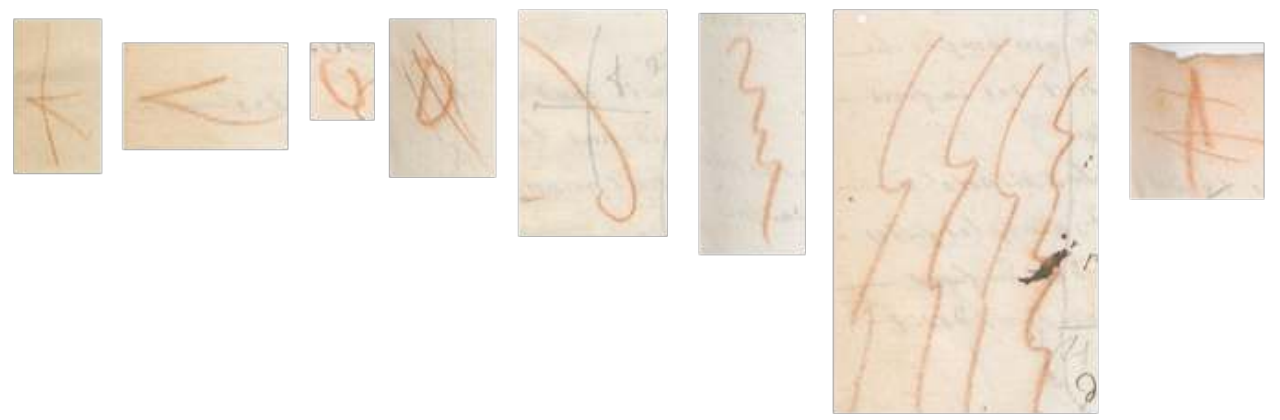
Fausseté des autres religions : p. 105 v $^{\circ}$ (Sel. 239)

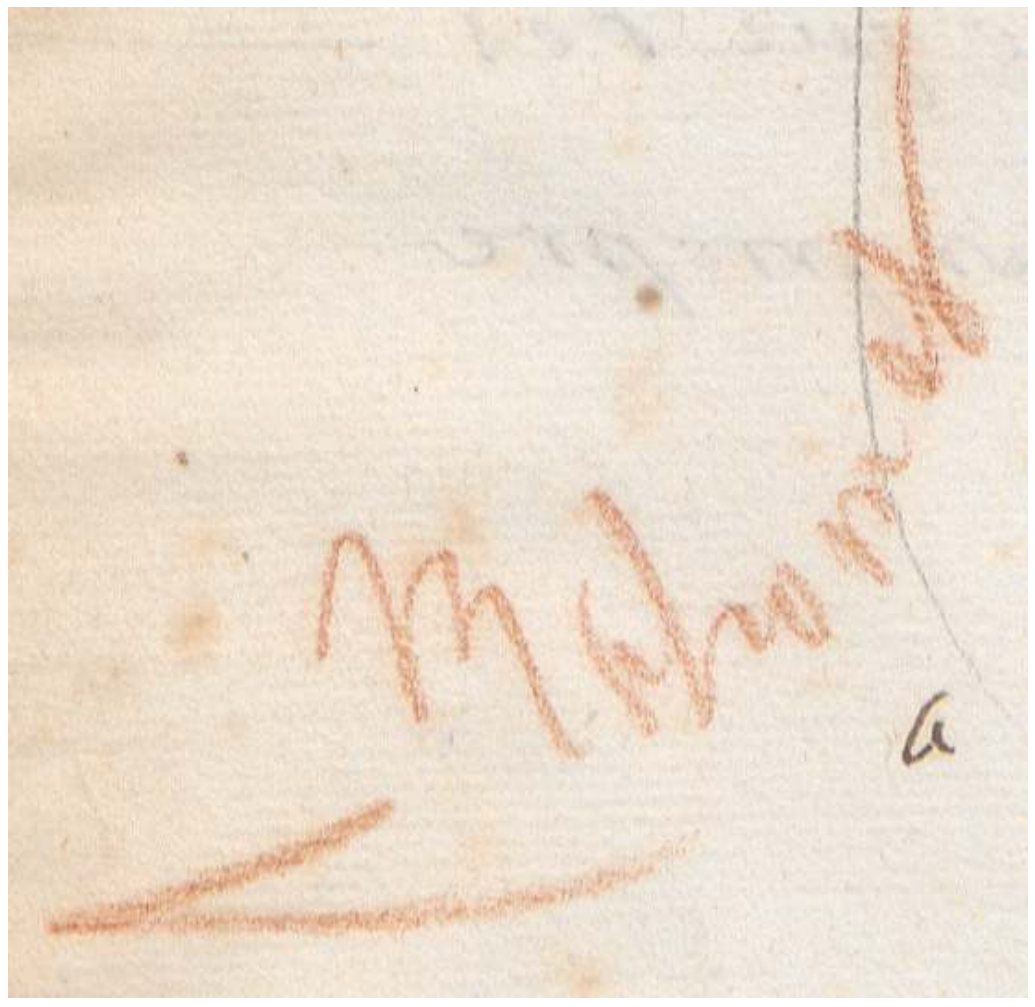

Un signe < suivi de de Mahommet a été écrit à la sanguine. Une partie du texte a été intégré en 1670 dans le chap. Contre Mahommet.

Ces signes sont parfois accompagnés d'un commentaire souvent en relation avec le chapitre de l'édition dans lequel ils ont été vus. Voir le détail en Annexe.

Liasses et dossiers concernés : Vanité, Misère, Raisons des effets, Philosophes, Souverain bien, Soumission, Fausseté des autres religions, Loi figurative, Preuves de Moïse, Prophéties, Morale chrétienne, I, II, III, IX, XI, XII, XIX, XXIII, XXV, XXVI, XXXIII, XXXIV. 


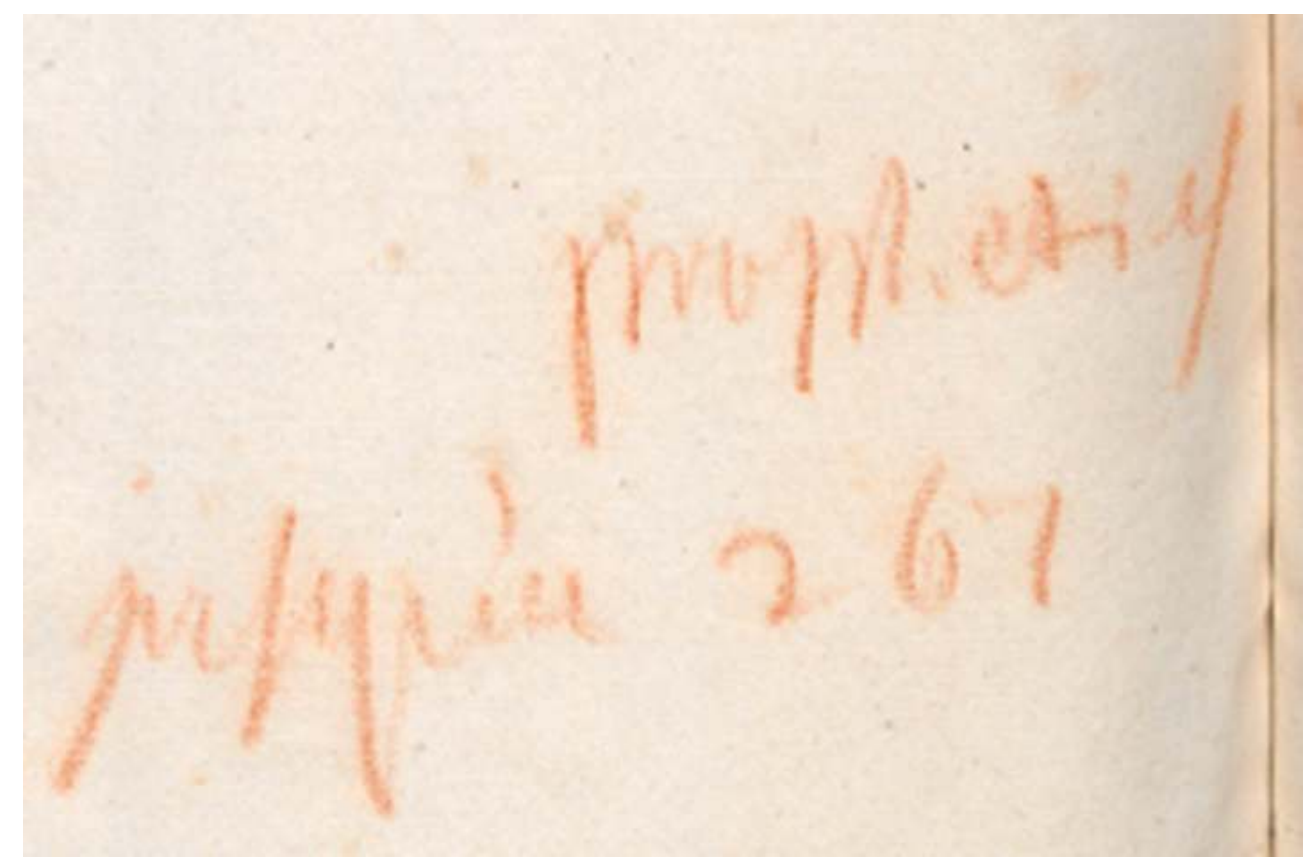

\section{Phases de sélection}

Une deuxième phase, qui alterne sanguine et encre noire, a consisté à marquer par des lettres $\mathrm{M}, \mathrm{B}$, ou R certains fragments ou paragraphes non encore retenus dans l'édition.

Quelques précisions ont aussi été signalées au cours de cette phase.

Lettres d'interprétation problématique : $M, B, R$.

P. 177v (Morale chrétienne - Sel. 245), le commentaire Commencement des membres pensans, voyez aux 4. pages suivantes, ajouté à l'encre noire, correspond à une refonte du chapitre Pensées morales. Plusieurs textes qui suivent seront ajoutés dans l'édition sans que d'autres marques le précisent.

P. 227 (unité IV - Sel. 690) : l'instruction le joindre au suivant a été ajoutée à l'encre noire. Cette instruction correspond à celle de la p. 227v, le joindre au precedent, qui n'a pas été suivie d'effet.

P. 317 (unité XXI - Sel. 392) : un B mis en valeur dans un cercle a été ajouté en marge et suivi de l'instruction Il faut adjouter les 2. lignes suivantes a la pensée qui commence par ces mots Il y en a qui masquent chap. Des pensées diverses page $3381^{\text {ere }}$ Edition. Cette instruction a été suivie d'effet. 


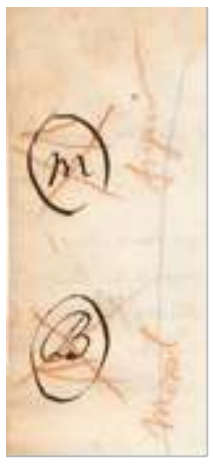

Un signe $<$, suivi de figure a été écrit à la sanguine devant un premier paragraphe ; un signe $<$, suivi de moral. a été écrit à la sanguine devant un deuxième paragraphe. Ces deux paragraphes n'ont pas été retenus dans l'édition de 1670. Ces indications ont été écrites en premier $^{98}$.

Les marques $\mathrm{M}$ et $\mathrm{B}$ écrites à l'encre noire et entourées d'un cercle de même couleur ont été ajoutées dans un deuxième temps.

Enfin, ces marques ont été cochées à la sanguine. Le premier paragraphe a été intégré dans l'édition 1678 dans le chap. Figures. Seul le début du deuxième paragraphe a été intégré en 1678 dans le chap. Pensées chrétiennes.

En fait, cette phase est un peu plus complexe et peut se décomposer en quatre étapes :

a. Les marques sont notées à la sanguine sans les entourer d'un cercle de la page 1 à 122 (unités Ordre ( $\mathrm{M}$ et $\mathrm{B})$, Vanité $(\mathrm{M})$, Misère ( $\mathrm{M}$ et $\mathrm{B})$, Raisons des effets (R), Divertissement $(\mathrm{M})$, Commencement (M), et Fondements (M)).

b. Des marques sont ajoutées à l'encre noire et entourées d'un cercle dans Raisons des effets (R), Commencement (M), Transition (B et R), Fausseté des autres religions (B), Loi figurative (M et $\mathrm{B})$, Preuves de J.-C. (M), Morale chrétienne (B), unités I (M et B), II (M), III (B), IV (B), V (B), XI (M et B), XVIII (B), XIX (B), XX (B), XXI (B), XXII (B), XXIII (M et B), XXIV (B) et XXV (M et B). La marque $R$, écrite à la sanguine dans l'étape précédente dans Raisons des effets et la marque $M$, écrite à la sanguine dans l'étape précédente dans Commencement, ont été repassées à l'encre noire puis cerclées.

c. Certaines des marques ajoutées dans l'étape 2 ont été cochées à la sanguine.

d. À partir de la page $381 \mathrm{v}^{\circ}$ (dernier $\S$ de la série XXV) toutes les marques ( $\mathrm{M}$ et B) sont à la sanguine et l'écriture des $B$ semble différente du reste. Toutes les marques sont dans un cercle rouge et certaines ont été cochées.

Lettres barrées d'interprétation problématique : B M
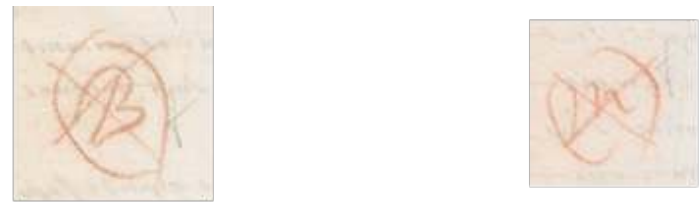


\section{Résultats de cette sélection} $\mathrm{J}$. Mesnard $(\mathrm{B}=\mathrm{Bien}, \mathrm{M}=\mathrm{Mal}, \mathrm{R}=\mathrm{A}$ rejeter), ne paraît pas conforme à ces résultats : les cinq fragments marqués $M$ et ajoutés à l'édition (chap. Figures $n^{\circ} 5$, Jésus-Christ $\mathrm{n}^{\circ} 6$, Pensées chrétiennes $n^{\circ} 64,75$ et 95) n'ont pas plus été modifiés que ceux marqués B. Seuls les fragments de la liasse Raisons des effets, auxquels il faut ajouter un texte barré de Disproportion de l'homme "Voilà une partie des causes qui rendent l'homme si imbécile [...] il est composé de deux natures différentes » (Sel. 230), ont été marqués d'un R. Aucun élément ne permet d'affirmer qu'une autre personne qu'Étienne soit intervenue sur la Copie à ce niveau. Cela d'ailleurs n'empêche pas que d'autres personnes aient pu participer à cette sélection (Blaise ? Marguerite ?). Étienne a pu servir de secrétaire. De plus, cette sélection est plus positive que ne le suggère P.-L. Couchoud: ce n'est pas le travail d'un censeur mais plutôt celui de la famille de Pascal qui avait le souci d'intervenir au minimum sur le texte publié.

\section{Pagination, numérotation des textes et concordance avec le Recueil des originaux dans $\mathrm{C}_{1}$}

\section{La pagination}

212 La pagination a était ajoutée à la plume sur le recto des feuillets (pages impaires). Les chiffres ne sont pas suivis d'un point. 


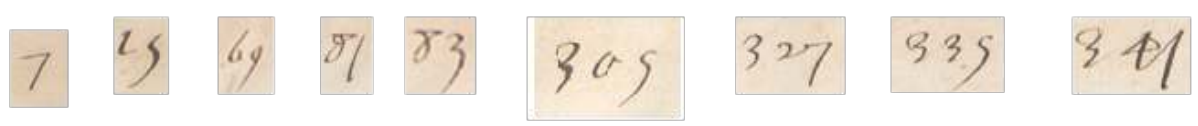

$7,15,69,8193,305,327,335,341$

Rares sont les pages qui ont été paginées au verso. Ces ajouts peuvent correspondre à des interventions ponctuelles sur le texte. Parmi ces pages, la page 122 a été paginée au crayon par une autre main (voir l'étude de la concordance ci-dessous). C'est peut-être aussi le cas des pages 10,12 et 22 dont le numéro a été mis dans une sorte de parenthèse.

Des oublis ont amené de nouvelles corrections: le plus caractéristique est celui des feuillets numérotés $8^{1}, 9^{1}$ et $8^{2}, 9^{2}$. Deux feuillets n'avaient pas été numérotés entre les pages $7 \mathrm{v}^{\circ}$ et 11. Les chiffres sont de la même main que ceux de la concordance (voir cette étude). Autre cas : le no 326 est de la même main que certains numéros de textes écrits au crayon (voir cette étude). Cette numérotation contient aussi des hésitations (exemple: 16116153 ) et des doublons (exemple : 37 bis, bis a été ajouté au crayon).

Ces numéros ont été suivis d'un point sauf $9^{2}$.

Numéros pairs correspondant à la pagination.
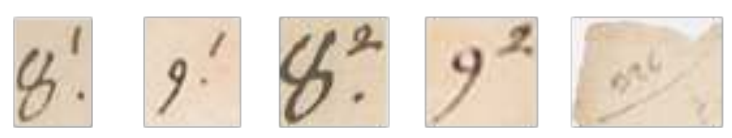

$8^{1}, 9^{1} 8^{2}, 9^{2} 326$

\section{Numérotation des textes}

Un numéro a été ajouté devant certains paragraphes dans le retrait de la première ligne. Cette numérotation ne tient pas toujours compte des papiers et des titres de liasses ${ }^{102}$.

Les tables des titres ne portent aucun numéro. L'identité de teinte entre l'encre utilisée par le copiste et la numérotation des textes est troublante et pourrait faire penser que ces numéros ont été écrits en même temps que le texte par le copiste. La comparaison des écritures montre qu'il n'en est rien, d'ailleurs certains numéros ont été écrits plus hauts dans l'interligne (par exemple, p. 197). La numérotation est continue et suit l'ordre de la Copie.

\section{Unités à titres}

Numéros écrits au crayon

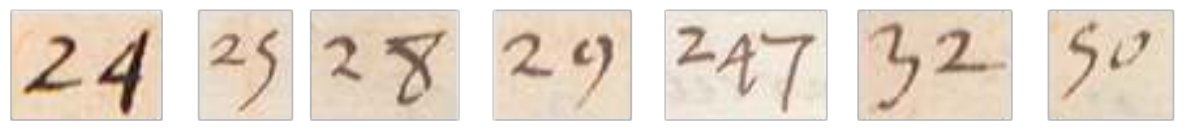

$24,25,28,29,247,32,50$ 
Numéros écrits au crayon dans l'interligne

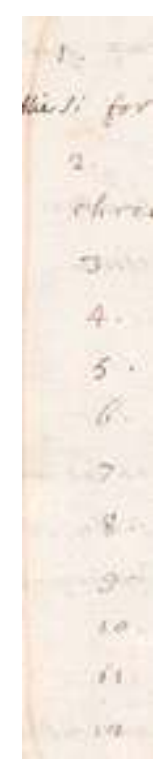

Copiste p. 258

Des numéros ont été écrits au crayon p. 121 à 154 (où se situe le papier original) sauf les numéros 315 et 316 p. 137 v , puis p. 177 vo-179. La numérotation tient compte du no 334 qui a été écrit sur le papier original ajouté à la Copie. P. $121 \mathrm{v}^{\circ}$, un numéro de texte est suivi du numéro de page dans le RO.

Numéros écrits au crayon

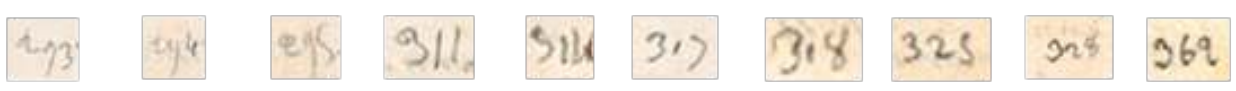

Autres dossiers :

Numéros écrits au crayon

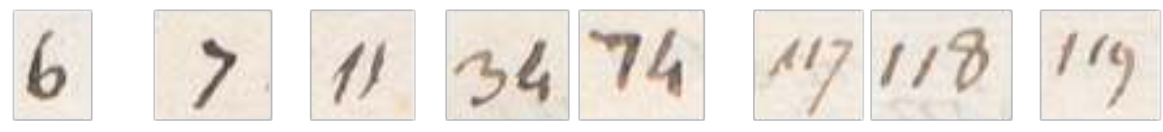

$6,7,11,34,74,117,118,119$

221 Les numéros ont été écrits au crayon p. 259 bis à $267 v^{0} ;$ p. 305 à 337 vº p. 423 jusqu’à la fin de la Copie.

Numéros écrits au crayon

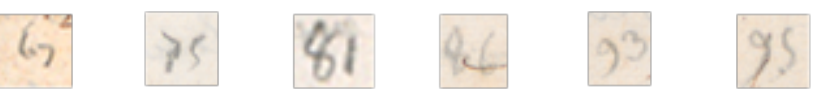

$67,75,81,86,93,95$

Courrier du Centre international Blaise Pascal, 32 | 2015 
Il ne semble pas que ce soit la même main qui écrive les numéros à la plume et au crayon. La main qui utilise le crayon a aussi ajouté la référence du papier à deux endroits. Elle aurait d'ailleurs pu le faire pour d'autres fragments, mais ce n'est pas le cas. Ses interventions au crayon pourraient être postérieures à la concordance. Certains numéros ont d'ailleurs été ajoutés dans l'interligne à cause de la concordance située à cheval sur le retrait de paragraphe (par exemple, $n^{\circ} 23$ et 24 p. 197). Il faut donc en conclure que la concordance et la numérotation des fragments ont été réalisées en parallèle.

$C_{1 p .} 197-n^{\circ} 23$

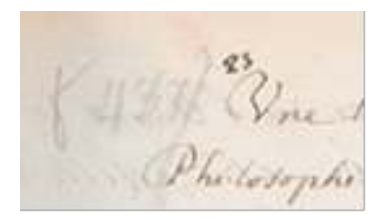

Les marques a, b, c, d, e, etc. ont aussi été écrites en même temps que cette numérotation ou juste après, et parfois dans la même encre. Certaines marques ont provoqué une modification de la numérotation, par exemple p. 197 et $197 \mathrm{v}^{\circ}$.

\section{La concordance entre le Recueil des originaux et la Copie $\mathrm{C}_{1}{ }^{103}$}

Une concordance entre ces deux documents a été écrite à la mine de plomb sur ces deux documents. Elle suppose que la Copie et le $R O$ ont été paginés et que les papiers étaient collés dans le $R O$, mais ne nécessite pas qu'ils aient été reliés.

Le premier à la mentionner est Augustin Renouard dans sa réédition des Pensées en 1812. Avertissement, p. xx : «Sans la copie, il seroit très difficile, sinon impossible, de faire usage de l'original; et encore, avec cette copie, on auroit bien du mal à s'y reconnoître, si la patience du P. Guerrier, religieux bénédictin ${ }^{104}$, qui l'a conservée et peut-être fait écrire, n'eût tracé dans l'un et l'autre volume des indications qui en sont la clef respective. Ces indications, dont on ne peut connoître l'emploi qu'après un certain examen, consistent en chiffres tracés à la mine de plomb, qui, dans la copie, font connoître les pages de l'original où on les peut trouver, et réciproquement, dans l'original, indiquent les pages où ils sont dans la copie ; avec cette différence cependant, que tous les chiffres, qui dans l'original sont précédés d'un tiret, de cette manière, - 85 , n'ont aucun rapport avec la copie, et qu'il ne faut consulter que les chiffres non accompagnés d'autres marques. ». Renouard se trompe lorsqu'il dit que c'est Jean Guerrier qui a fait écrire la Copie. Il est en fait influencé par le texte manuscrit que Jean Guerrier a écrit au début de la Copie. Mais peut-être a-t-il raison de penser que c'est Jean Guerrier qui a établi cette concordance. En effet, Jean Guerrier est un cousin de Louis et Marguerite Périer. Louis avait hérité du RO et Marguerite de $C_{1}$ et $C_{2}$ en 1687 à la mort de leur mère (Étienne est décédé en 1680 et Blaise en 1684). C'est à Jean que Marguerite donnera la Copie $\mathrm{C}_{1}{ }^{105}$. Pour établir la concordance entre les papiers originaux et la Copie, il fallait que ces deux documents soient disponibles en même temps et qu'une personne soit capable de lire les papiers de Pascal. Aucun écrit ne permet de le vérifier mais rien ne s'oppose à ce que Jean Guerrier ait travaillé avec Louis Périer, et éventuellement que Marguerite ait participé à ce travail. 
Dans $C_{1}$

La concordance dans $\mathrm{C}_{1}$ consiste en une sorte d'accolade écrite dans la marge de gauche, qui signale où commence et finit chaque texte, et le numéro de page du Recueil des Originaux où se situe le papier correspondant, lorsque celui-ci existe. Aucun commentaire n'a été ajouté si le papier est absent du Recueil ou si le texte n'est pas visible (cas de textes redécouverts au verso de certains papiers). Le numéro de page du $R O$ n'est pas répété si un nouveau papier est collé sur la même page que le précédent et ce changement de papier est seulement signalé par une sorte de « 8 ».

$C_{1 \mathrm{p} .} 341$

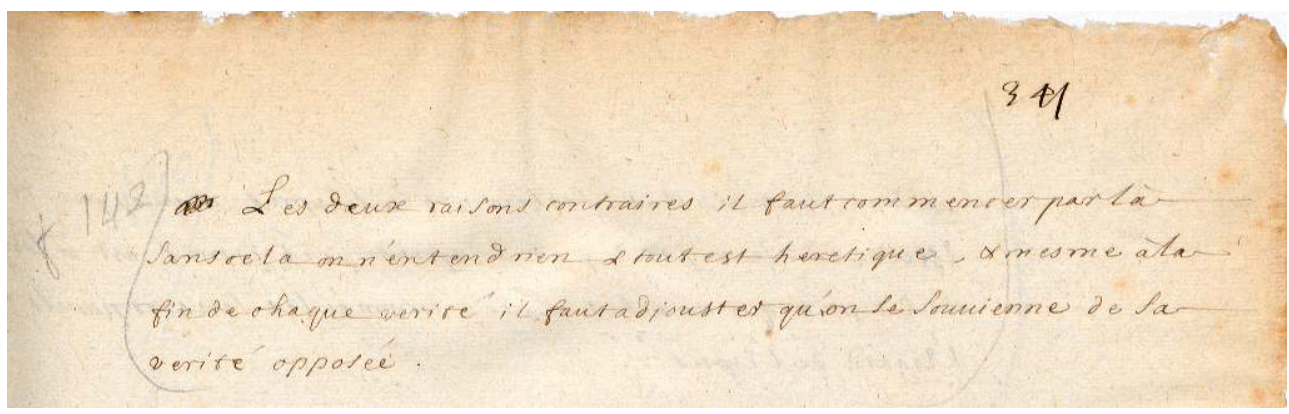

$R O$ p. $142\left(n^{\circ} 341\right.$ à gauche du papier)

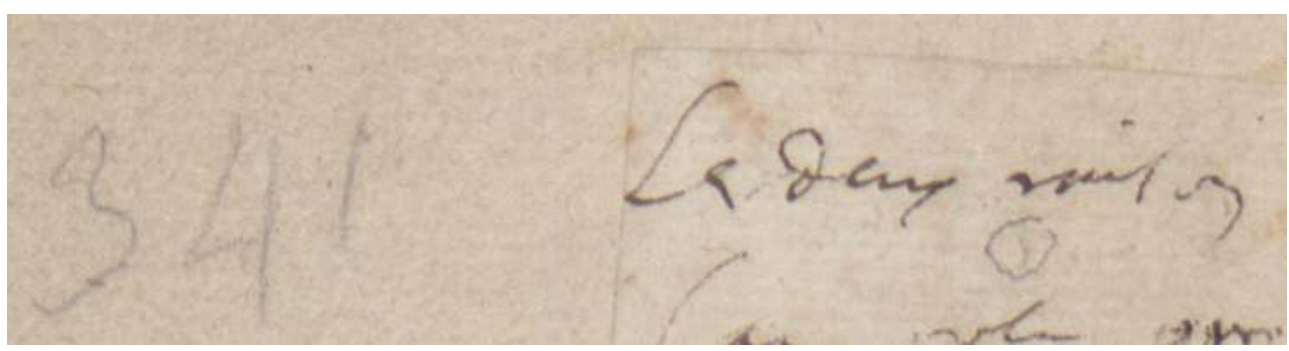

Les accolades dans $\mathrm{C}_{1}$ tiennent compte à la fois des lettres $\mathrm{a}, \mathrm{b}, \mathrm{c}, \ldots$ ajoutées en marge et des numéros donnés à ces textes. La concordance a été réalisée en parallèle avec la numérotation des textes (voir ci-dessus) ${ }^{106}$. D'autres chiffres ont été ajoutés p. 121 (132 138 - 127 à droite) et 122 ( 83 à gauche) mais ont été écrits par une autre main ${ }^{107}$.

\section{Dans le RO}

Un numéro a été ajouté à gauche de la plupart des papiers, exceptionnellement sur le papier et encore plus rarement à droite par manque de place (mais sans tiret). La grande majorité de ces numéros correspondent aux numéros des pages de $C_{1}$ dans lesquelles sont transcrits les textes des papiers, une page paire, non paginée dans $C_{1}$, étant signalée par un numéro pair. Une partie de ces numéros (de 1 à 7 sauf 4) est précédée d'un $\mathrm{P}^{108}$. Certains sont écrits sur les papiers, d'autres à cheval sur le support et le papier. Il semble que la personne qui a commencé ainsi a été arrêtée par le fait que le feuillet qui suit la page $7 \mathrm{v}^{\circ}$ n'était pas paginée (voir ci-dessus).

Certains de ces numéros sont en partie dans la reliure ou ont été tronqués lors de la confection de cette reliure. 
230 C'est la même main qui a noté les références sur les deux documents. C'est elle aussi qui a écrit des chiffres à droite des papiers et précédés d'un tiret, mais dont le sens n'est pas éclairci.

Échantillon de chiffres dans $\mathrm{Cl}$

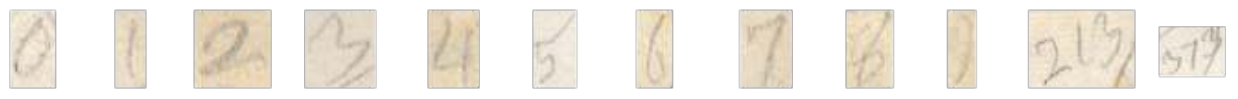

$0,1,2,3,4,5,6,7,8,9,213,373$

Échantillon de chiffres dans le $R O$
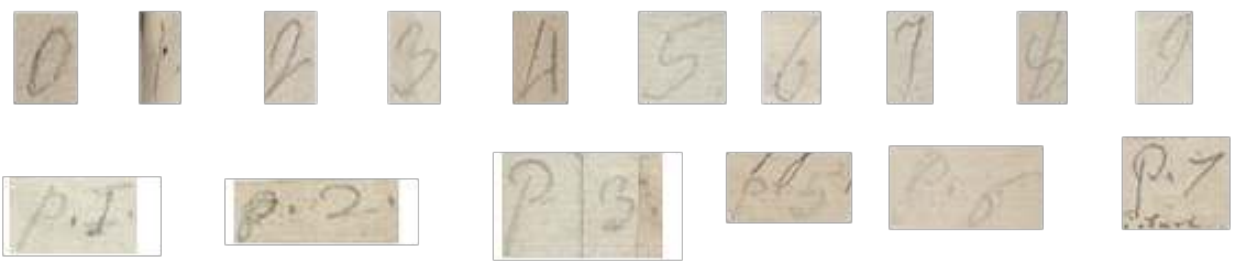

$0,1,2,3,4,5,6,7,8,9$

p. 1, p. 2,p. 3, p. 5, p. 6, p. 7

231 Ces chiffres sont parfois accompagnés de quelques mots dans C1:

jusqu'a $13^{109}$

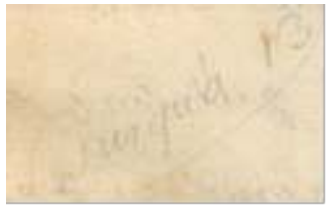

C1 p. $8^{2}$

p[aragraphe] general

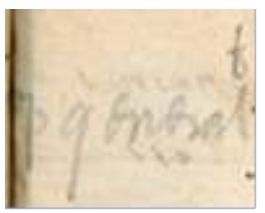

$\mathrm{C}_{1 \mathrm{p} .} 13$

facheux

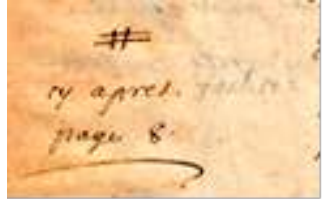

C1 p. $17 v^{\circ}$ 


\section{Pagination et numérotation des cahiers du $R O$}

La pagination a été faite avec beaucoup de soin au recto et au verso des feuillets, y compris sur les nombreuses pages blanches. Les chiffres sont suivis d'un point. C'est la même main que celle qui a noté la concordance.

Exemples de pagination
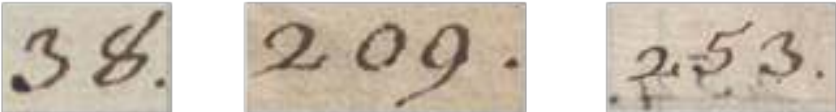

$38,209,253$

L'encre utilisée pour numéroter les cahiers est un peu plus claire. Les numéros ne sont pas suivis d'un point. La forme des chiffres est complètement différente, notamment les 8 et les 5 :

Exemples de numérotation

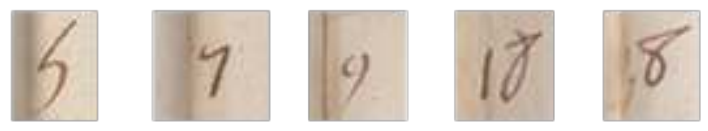

$5,7,9,18,8$

\section{Conclusions}

L'étude comparative des écritures des chiffres dans $C_{1}$ et le $R O$ révèle que les numéros tracés dans $\mathrm{C}_{1}$ - pour paginer la Copie, numéroter des textes et établir la concordance avec le $R O$ - et le $R O$ ont été écrits par deux personnes :

Main $\mathrm{n}^{\circ} 1 /$ - Concordance dans $\mathrm{C}_{1}$; concordance dans le $R O$; numéros à droite des papiers dans le RO; et peut-être P.1 à P.7; le tout à la mine de plomb. À cela s'ajoutent la numérotation des pages $8^{1}$ et $8^{2}$ dans $C_{1}$ et la numérotation des pages du RO, les deux à la plume et suivis d'un point.

Concordance dans $\mathrm{C}_{1}$
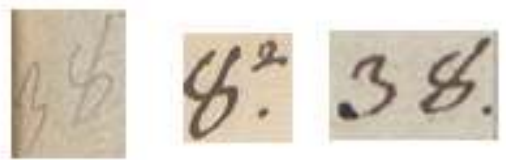

RO p. 191 - p. $8^{2}$ dans $C_{1}$ RO p. 38 
Main $n^{\circ} 2 /$ - Numérotation des cahiers du $R O$; numérotation des textes dans $C_{1}$; pagination de $C_{1}$; le tout à la plume.

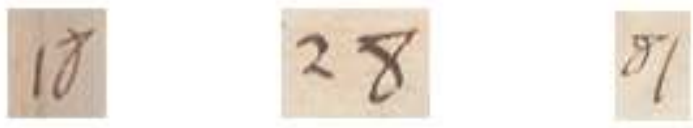

$\mathrm{N}^{\circ}$ cahier du $R O-\mathrm{n}^{\circ}$ texte dans $\mathrm{C}_{1}$ - p. 81 dans $\mathrm{C}_{1}$

On retrouve la main $\mathrm{n}^{\circ} 2$ dans le commentaire vide $198 \mathrm{p}$. en marge de $C_{1 \mathrm{p}}$. 33 , ainsi que sur trois machines de Pascal (mêmes 8 et 5 sur celles de Durant-Pascal et du CNAM; même 7 sur une machine du musée du Ranquet).

C1 p. 53

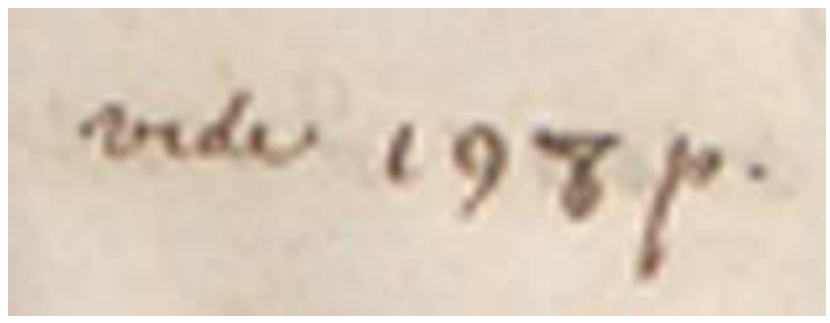

Pascaline C.N.A.M

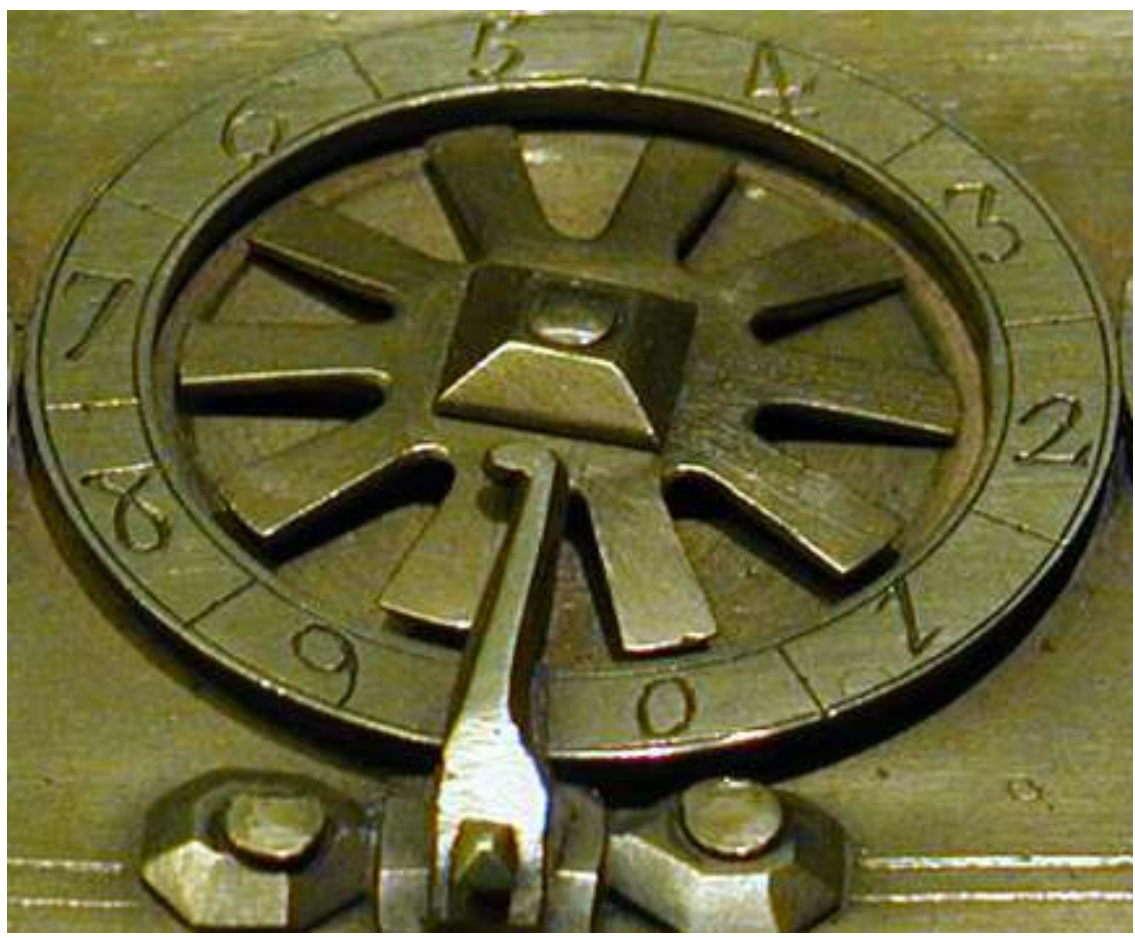

\section{Échantillons d'écriture}




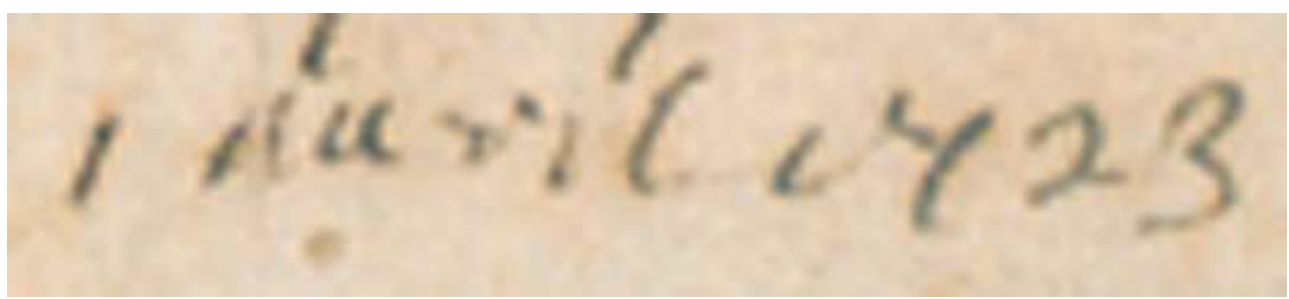

Louis Périer, copie du Mémorial et attestation

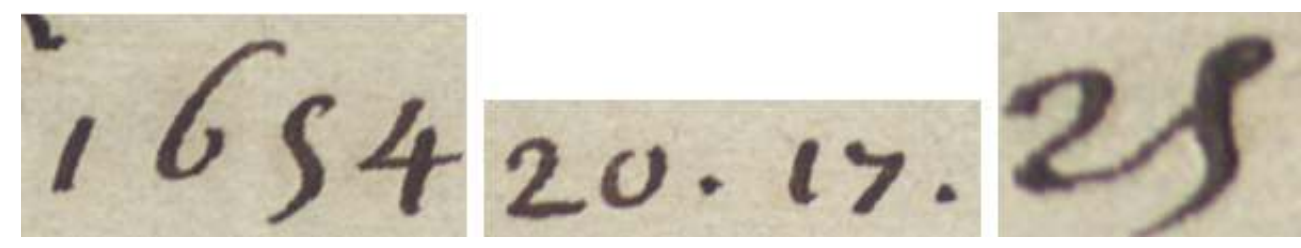

Marguerite Périer (Formulaire de don des Lettres d'Amos Dettonville à l'oratoire de Clermont) : (15 mars 1723) $)^{110}$

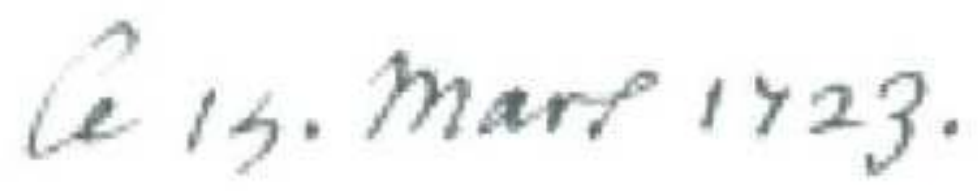

Les chiffres 7 et 2 sont semblables à ceux de Jean Guerrier.

\section{Hypothèse}

Louis Périer a paginé $C_{1}$ avant la phase de repérage des textes par Étienne pour l'édition de $1678^{111}$. Dans un deuxième temps, après le collage des papiers dans le RO, Louis Périer s'est associé à Jean Guerrier pour en faciliter la lecture : il a numéroté une partie des textes et laissé des marques de classement $\left(a, b, c, d\right.$, etc.) dans $C_{1}$. Il a numéroté les cahiers du RO.

Jean Guerrier a paginé le $R O$ (avec une plume et en s'appliquant) ${ }^{112}$ puis a établi les concordances $C_{1} / R O$ et $R O / C_{1}$ en alternance avec Louis Périer qui numérotait les textes dans $C_{1}$ : Jean Guerrier a commencé par lire $C_{1}$ en écrivant P.1, ..., P.7 sur le RO mais a été arrêté par deux pages numérotées 9 dans $C_{1}$. Il a alors numéroté les deux pages 8 (à la plume) et a continué la concordance en abandonnant les P. Jean Guerrier a utilisé une mine de plomb sauf pour la pagination.

Cette étude demanderait évidemment de réunir d'autres documents contenant des chiffres écrits de la main de ces trois personnes.

\section{Marques et corrections diverses dans $C_{1}$}

vide 198 p., p. 53 : ce commentaire a été ajouté en marge du texte (Sel. 165) «Si l'homme estoit heureux il le seroit d'autant plus qu'il seroit moins diuerty comme les Saincts et Dieu. ». Le sens du commentaire serait voir p. 198 qui pourrait être un renvoi au texte « La 
seule chose qui nous console de nos miseres est le divertissement [...] » (Sel. 33). Ce renvoi a été écrit après la pagination de $C_{1}$ et pourrait être de Louis Périer (voir ci-dessus).

Liasse Soumission: trois mots, non retenus dans l'édition, ont été ajoutés p. 81 (Sel. 201); Mais a été ajouté au-dessus du numéro de texte ce qui supposerait qu'il a été écrit après. Mais est aussi précédé d'un 2 de la même encre. Le deuxième ajout peut se lire dit lisentius ; Licentius est un poète latin et disciple de saint Augustin. L'écriture n'est pas identifiée.

Mais 2. 228. II faut

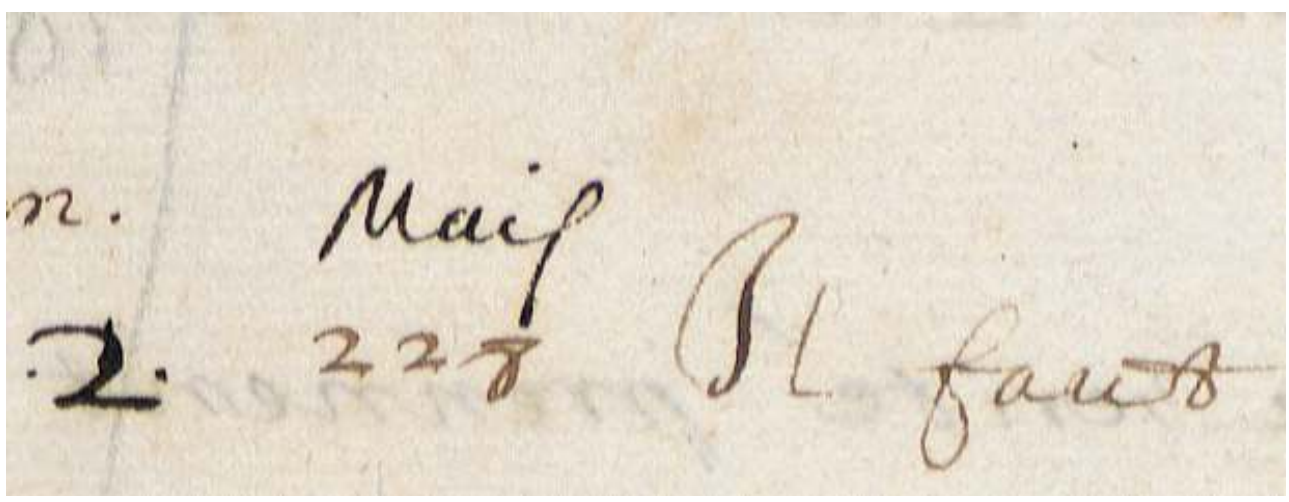

C $_{1}$, Liasse Soumission, p. 81 (Sel. 201)

Dit lisentius

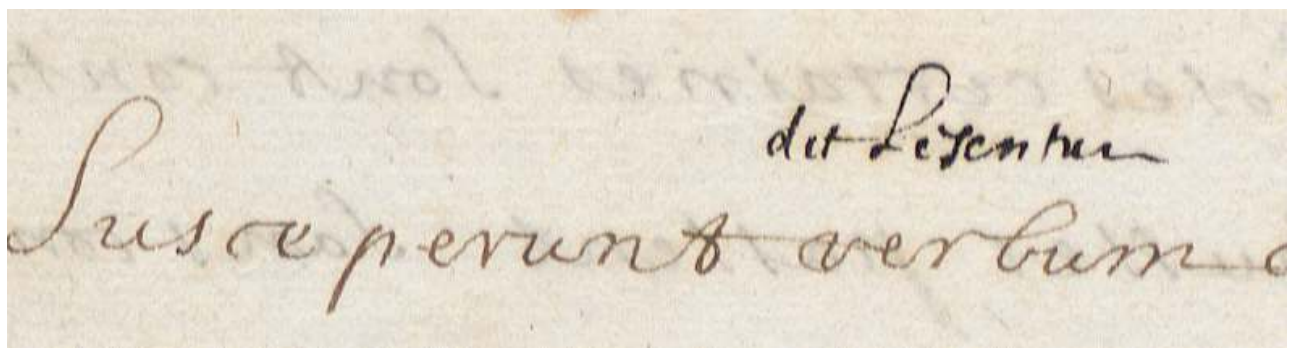

C ${ }_{1}$ Liasse Soumission, p. 81 (Sel. 201)

De grandes croix ont été tracées en marge au crayon dans les pages $1 \mathrm{v}, 5,11,15,15 \mathrm{v}, 22^{113}$, $31,33 \mathrm{v}, 37 \mathrm{v}, 39 \mathrm{v}, 53,77 \mathrm{v}, 81 \mathrm{v}, 89,91,93 \mathrm{v}, 95,97,121$ et $121 \mathrm{v}^{114}$. Elles correspondent à autant de fragments déjà publiés en 1844 sous leur état original, y compris dans le Rapport de V. Cousin (1843). Elles sont probablement dues à Faugère.

C'est aussi Faugère qui ajoute une croix au crayon et numérote un fragment 111' p. $31 \mathrm{v}^{\circ}$ (Sel. 118) après avoir constaté qu'il y avait un changement de papier (perdu - puis redécouvert en 1843). C'est probablement lui qui entoure 0 ridicolossimo Heroe p. $13 \mathrm{v}^{\circ}$ (Sel. 81), signale un fragment par une croix dans un losange p. 53 (Sel. 168), etc.

L'abréviation In. (et non $m$.) a été notée à la plume, dans la marge en face du fragment $\mathrm{n}$ - 192 p. 445 (Sel. 427). Elle pourrait signifier Inédit ; en effet ce texte était dans la copie Sainte-Beuve mais n'avait pas été publié avant l'édition Faugère. Les commentaires p. 451 et in p. 439 (Sel. 421) renvoient à un fragment marqué Com (ou bom ? bon?) p. 451 (Sel. 433). Ces deux fragments sont dans l'édition de Port-Royal et n'ont été publiés dans leur état original qu'en 1844. La référence p. 453 située en face d'un texte ajouté par Nicole p. $445 \mathrm{v}^{\circ}$ renvoie à un fragment de la Copie p. 453 (Sel. 435) dans lequel les textes sont 
terminés par lequel est le plus clair. Toutes ces marques sont d'une même main qui pourrait aussi être celle de Faugère.

\section{La Copie $C_{2}$ : une copie (presque) épargnée}

245 Contrairement à $C_{1}$, cette Copie ne contient aucune trace de son utilisation dans la préparation de l'édition de Port-Royal. En revanche, la copie Sainte-Beuve, copie figurée du ms Périer selon J. Mesnard, montre que Louis Périer a largement puisé dans cette Copie.

\section{Une copie de Pierre Guerrier p. 399 à 401 v}

Le texte transcrit dans $C_{2 \text { p. }}$ 399-401 vo (un cahier composé d'une feuille écrite recto-verso) est surprenant à plus d'un titre. Contrairement au reste de la Copie, l'écriture n'est pas celle du copiste habituel mais celle de Pierre Guerrier. La copie contient une retranscription du dossier XVI entre les dossiers XXX et XXXI. Ce texte est pourtant transcrit p. 507 à 509 . Cette copie transcrit ensuite le dossier XXI. La dernière page de cette copie transcrit le début du dossier XXII en s'arrêtant au premier quart de la page, le texte continuant ensuite dans un nouveau cahier avec l'écriture du copiste habituel.

J. Mesnard dans Les «pensées » ont trois cents ans, p. 10 : «ce manuscrit est uniformément composé de cahiers de huit feuillets. Exception toutefois pour le $28^{\mathrm{e}}$ de ces cahiers (dans l'ensemble du recueil $\mathrm{RC}_{2}$, il s'agit du cahier 30) (pages 419 à 426), qui comporte seulement quatre feuillets, mais il s'agit en fait du résidu d'un cahier de huit feuillets, comme le prouvent l'examen des filigranes, auxquels manquent leurs correspondances, et l'existence de quatre onglets. Autre exception pour le $26^{\mathrm{e}}$ cahier $\left(\mathrm{RC}_{2}\right.$, cahier $\left.\mathrm{n}^{\circ} 28\right)$, celui que le P. Guerrier a rédigé de sa main et qui se compose de deux feuillets seulement. À notre avis, le texte que le $P$. Guerrier a recopié figurait sur les quatre feuillets détachés du cahier aux onglets et qui ne pouvaient entrer commodément dans la reliure. Il y a tout lieu de croire qu'il a mis sa copie à la place où devaient se trouver normalement les feuillets qu'il a reproduits. »

La copie de P. Guerrier, probablement établie après 1731 (date de son entrée à l'oratoire de Clermont) ne conserve pas la mise en page habituelle de la Copie $C_{2}$ (marges, interlignage) et contient des fautes qui ne sont ni dans $C_{1}$ ni dans $C_{2}$ (unité XVI p. 507-509). De plus, P. Guerrier n'a pas toujours reproduit les références bibliques situées en marge ou à part, ni l'accolade, ni la note située en marge : "Vous ne serez plus mon peuple \& je ne seray plus vostre dieu, apres que vous seres multipliez de la dispersion. Les lieux ou l'on n'appelle pas mon peuple je l'appelleray mon peuple. »

Pierre Guerrier a écrit en marge de ce texte «il faut mettre tout ce qui suit dans une feuille separée.»

\section{La série XVI avait-elle été copiée à ce niveau, entre les unités XXX et XXXI, par le copiste habituel de $\mathrm{C}_{2}$ ?}

250 La transcription des textes concernés par la copie Guerrier selon les critères du copiste habituel aurait demandé six feuillets écrits recto-verso. En revanche, la transcription des mêmes dossiers sans le dossier XVI et sans changement de page entre les unités utiliserait quatre feuillets écrits recto-verso. Ces quatre feuillets, qui formaient la moitié du cahier 
28, pourraient correspondre aux feuillets montés sur onglet dans le cahier 30. La transcription du dossier XVI par le copiste habituel a été faite sur le même cahier $\left(\mathrm{n}^{\circ} 36\right)$ que l'unité XVII et n'en a pas été séparée. Le dossier XVI transcrit par Pierre Guerrier n'était donc pas la transcription du copiste habituel. $321 \mathrm{v}^{\circ}$ ) ou parfois d'un trait (p. 167, 219, 305, 309). Parfois la marque est $J$ et $N$ ou $J$ \& $N$ ou $J$ N ou N. J. Les liasses Rabbinage, Figures particulières, et les dossiers XXXV et XXXII ne sont pas concernés. Ce marquage s'arrête brusquement p. 327 (au milieu du dossier XXV) sauf p. $423 \mathrm{v}^{\circ}(\text { un } J)^{115}$. 
D'autres marques correspondent probablement à la même phase de marquage : cy devan pag 76 et 77 p. 82 ; Tous (Tout ?) a examiner écrit en haut de la page 179 ; quatre feui. plus loin p. 261 ; quatre feuill. avant p. 269, ainsi que de grandes croix (certaines sont barrées p. 278, 280 - d'autres non p. 280, 283 - ou multiples p. 282 ou en forme de X p. 289 v $^{\circ}$ ). Les références quatre feui. plus loin et quatre feuill. avant correspondent à deux paragraphes dont le thème est le schisme (Sel. 442 et 450). Le commentaire ci-devant p. 76 et 77, ajouté dans la marge d'un fragment titré Divertissement (Sel. 169) fait référence au plus grand fragment sur le divertissement (Sel. 168).

Tous (Tout ?) a examiner

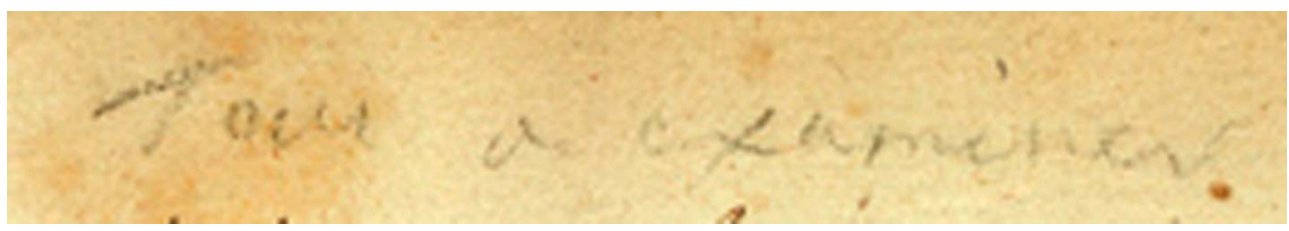

Écrit en haut de la page 179

\section{Autres traces}

P. 28 (Sel. 78), des l'enfance a été corrigé de l'enfance en barrant le $s$ : cette correction suppose une relecture du papier original $\operatorname{car} \mathrm{C}_{1 \mathrm{p} .} 11$ et $\mathrm{C}_{2}$ avaient transcrit des et l'original (RO 369-1) porte de. Dans le même texte, forte a été barré au crayon dans illusion forte. forte n'est pas sur le papier original.

P. 35 (Sel. 94), deux corrections utilisent une convention qui paraît très moderne :

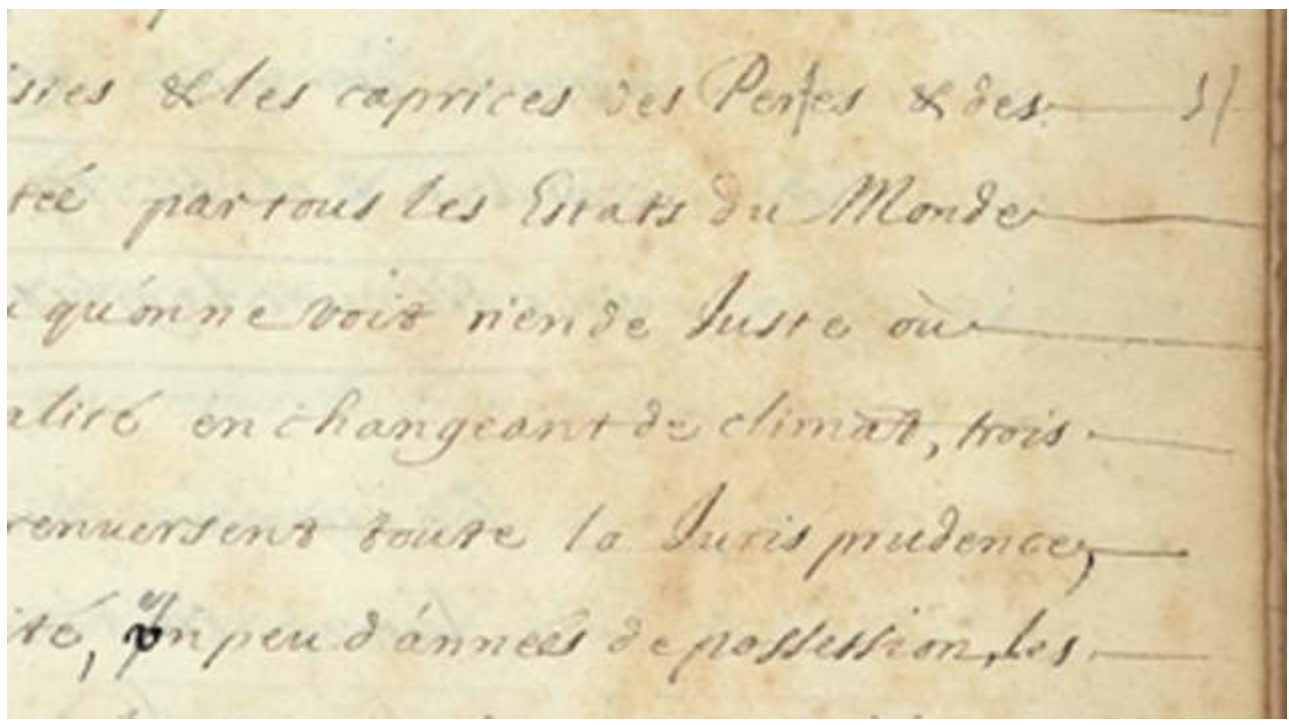

Ce fragment a été publié pour la première fois à partir du ms. Périer par Desmolets.

La première correction propose de remplacer Peres par Perses, conformément à $C_{1 p} .17$ et à l'original. Ce fragment a été publié pour la première fois à partir du ms Périer par Desmolets. La copie Sainte-Beuve et Desmolets transcrivent « perses ».

La deuxième propose de remplacer un peu d'années par en peu d'années : le crayon corrige le $u$ en $e$ conformément à $\mathrm{C}_{1}$ et à l'original. 
Date ajoutée au crayon sur la copie p. $1: 1760$.

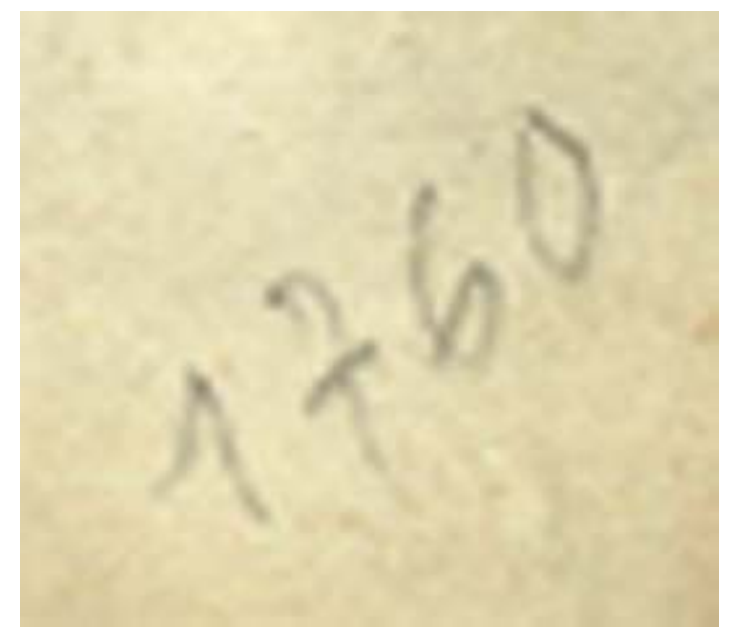

Date de réalisation de la reliure?

\section{La pagination}

269 J. Mesnard, OC, I, p. 294: «le P. Guerrier avait paginé le début de la seconde Copie (premier feuillet mis à part) p. 1-215. Cette numérotation, qui laissait pourtant de côté les toutes premières pages, a été conservée, et poursuivie de plusieurs mains, qui généralement n'ont porté que les numéros impairs. "

270 J. Mesnard concluait en $1971^{116}$ : «C'est dire aussi qu'il faut envisager les Pensées, non comme faites, mais se faisant, y voir un mouvement brusquement interrompu, ce qui oblige à une sorte de lecture dans le temps, de lecture en relief. L'effort est rude pour le public. L'éditeur, à l'avenir, ne pourrait-il considérer que sa principale tâche est de la faciliter? " L'édition électronique que nous préparons tentera de répondre à cette question difficile en mettant en relation hypertexte plusieurs transcriptions modernes associées à des notes explicatives à plusieurs niveaux (textes, liasses, projet apologétique), ainsi qu'aux documents manuscrits, où les deux Copies prendront une place essentielle, et aux premières éditions. L'ensemble, structuré en trois niveaux : grand public, public avancé et spécialiste, proposera de nombreuses pistes notamment bibliographiques ainsi que l'état actuel des recherches papier par papier.

\section{ANNEXES}




\section{Ordonnancement détaillé des Copies}

\begin{tabular}{|l|l|}
\hline $\mathrm{C}_{1}$ & $\mathrm{C}_{2}$ \\
\hline
\end{tabular}




\begin{tabular}{|c|c|}
\hline $\begin{array}{l}\text { Unités à titres } 1 \text { à } 27 \text { (p. } 1 \text { à } \\
188)\end{array}$ & Table des titres puis unité $I$ (p. 0 à 12) \\
\hline Table des titres p. 0 & Table des titres p. 0 \\
\hline Ordre p. 1 & Unité I p. 1 \\
\hline Vanité p. 5 & Unités à titres 1 à 27 (p. 13 à 220) \\
\hline Misère p. 15 & Ordre p. 13 \\
\hline Ennui p. 27 & Vanité p. 17 \\
\hline Raisons des effets p. 31 & Misère p. 33 \\
\hline Grandeur p. 37 bis & Ennui p. 45 \\
\hline Contrariétés p. 45 & Raisons des effets p. 47 \\
\hline Divertissement p. 53 & Grandeur p. 57 \\
\hline Philosophes p. 61 & Contrariétés p. 65 \\
\hline Souverain bien p. 65 & Divertissement p. 75 \\
\hline APR p. 69 & Philosophes p. 85 \\
\hline Commencement p. 77 & Souverain bien p. 91 \\
\hline Soumission p. 81 & APR p. 95 \\
\hline Excellence p. 85 & Commencement p. 102 \\
\hline Transition p. 89 & Soumission p. 107 \\
\hline Fausseté autres religions p. 105 & Excellence p. 111 \\
\hline Religion aimable p. 113 & Transition p. 115 \\
\hline Fondements p. 117 & Fausseté autres religions p. 129 \\
\hline Loi figurative p. 125 & Religion aimable p. 139 \\
\hline Rabbinage p. 141 & Fondements p. 143 \\
\hline Perpétuité p. 145 & Loi figurative p. 151 \\
\hline Preuves de Moïse p. 153 & Rabbinage p. 171 \\
\hline Preuves de J.-C. p. 157 & Perpétuité p. 175 \\
\hline Prophéties p. 165 & Preuves de Moïse p. 183 \\
\hline Fig. particulières p. 173 & Preuves de J.-C. p. 187 \\
\hline Morale chrétienne p. 177 & Prophéties p. 197 \\
\hline Conclusion p. 185 & Figures particulières p. 207 \\
\hline Table des titres et unité I (p. 189 & Morale chrétienne p. 209 \\
\hline à 200) & Conclusion p. 217 \\
\hline Table des titres p. 189 & Unité XXXV (p. 221 à 228) \\
\hline Unité I p. 191 & Titre Miracle (p. 225) \\
\hline Unités II à XIX (p. 201 à 312) & Unités XXXII à XXXIV (p. 229 à 274) \\
\hline Unité II p. 201 & Unité XXXII p. 229 \\
\hline Unité III p. 209 & Unité XXXIII p. 235 \\
\hline Unité IV p. 221 & Unité XXXIV p. 254 \\
\hline Onurier du Centre international Blaise Pas & $\begin{array}{l}\text { cal, } 32 \text { | } 2015 \\
\text { Unités XXIII à XXXI (p. } 275 \text { à 401) }\end{array}$ \\
\hline Unité VI p. 233 & Unité XXIII p. 275 \\
\hline Unité VII p. 237 & Unité XXIV p. 303 \\
\hline
\end{tabular}




\section{Corrections proposées pour l'édition de 1670}

Des corrections, écrites à la plume, ont été proposées dans les cahiers suivants :

-Vanité: une dizaine de corrections identifiées par J. Mesnard comme étant celles de Nicole (Sel. 61, 67, 70, 78, 80). La régularité de l'écriture fait aussi penser à celle d'Arnauld. Plusieurs ont été prises en compte dans l'édition de 1670.

- Grandeur : une seule correction dans au lieu de à p. 37 bis vº identifiée par P. Faugère comme étant celle d'Arnauld (Sel. 141). La partie corrigée n'a pas été retenue dans l'édition.

- Contrariétés: trois corrections identifiées par P. Faugère comme étant celles d'Arnauld (Sel. 154, 164). La correction de la page 50 (Sel. 164) a été retenue dans l'édition de 1670.

- Philosophes : une seule correction dans nous au lieu de hors de nous p. $61 \mathrm{v}^{\circ}$ (Sel. 176), identifiée par M. Pérouse comme étant celle d'Étienne. Pourrait aussi être celle d'Arnauld. Cette correction a été prise en compte dans l'édition de 1670.

- Souverain bien: trois corrections p. 65 (Sel. 181) identifiées par P. Faugère comme étant celles de Nicole. Pourrait aussi être celle d'Arnauld (même écriture dans Vanité ). Ces corrections ont été prises en compte dans l'édition de 1670.

- Soumission : nombreuses corrections identifiées par P. Faugère comme étant celles de Nicole (Sel. 201, 205, 208, 212, 213, 214, 215, 218). Plusieurs ont été prises en compte dans l'édition de $1670^{118}$.

- Transition : huit corrections identifiées par P. Faugère comme étant celles d'Arnauld (Sel. 229, 230). La plupart ont été prises en compte dans l'édition de 1670.

- Fausseté des autres religions: deux interventions identifiées par P. Faugère comme étant celles d'Arnauld (Sel. 240, 242). Les deux ont été prises en compte dans l'édition de 1670.

- Religion aimable : les deux occurrences de l'expression Parum est ut \&c. Isaye ont été barrées et le mot Juifs a été ajouté en marge d'un texte p. 113 (Sel. 254). Écriture d'Arnauld ? Le fragment a été retenu dans l'édition 1670 sans ces expressions.

- Fondements : une seule correction p. $117 \mathrm{v}^{\circ}$ (Sel. 266) identifiée par P. Faugère comme étant celle d'Arnauld. La phrase sur laquelle est portée cette correction n'a pas été retenue dans l'édition.

- Loi figurative: une seule correction p. $139 \mathrm{v}^{\circ}$ (Sel. 307) identifiée par P. Faugère comme étant celle d'Étienne. Le texte ajouté font des discours qui paroissent bas a été pris en compte sous une forme légèrement différente dans l'édition de janvier 1670 p. 133 (voir la photo au début de la section).

- Prophéties : sept corrections p. 165, 165 v et 169 identifiées par M. Pérouse comme étant celles de Nicole (Sel. 355, 357, 359, 370). Toutes ont été prises en compte dans l'édition de 1670.

- Morale chrétienne : cinq corrections identifiées par J. Mesnard comme étant celles d'Étienne (Sel. 385, 391, 392, 395, 399). Seule celle qui a été portée p. 177 (Sel. 385) a été prise en compte dans l'édition de 1670.

- Unité II : huit corrections p. 203 (Argument du pari, Sel. 680) identifiées par M. Le Guern comme étant celles d'Arnauld, ce qui n'est pas évident. Seules deux de ces interventions ont été retenues dans l'édition. 
- Unité III : une seule correction p. 220 (Sel. 683), qui nous paraît être celle d'Étienne (même écriture que dans l'unité IV). Ce fragment et sa correction n'ont été intégrés dans l'édition qu'en 1678.

- Unité IV : une seule correction p. 221 vo (Sel. 686), identifiée par M. Pérouse comme étant celle d'Étienne. Ce fragment et sa correction n'ont été intégrés dans l'édition qu'en 1678.

- Unité V : une correction p. 225 Vº (Sel. 690), qui nous parait être celle d'Étienne. Ce fragment et sa correction n'ont été intégrés dans l'édition qu'en 1678.

- Unité XVI : deux corrections p. 285 (Sel. 734), identifiées par M. Pérouse comme étant celles de Nicole, ce qui n'est pas évident. Ce fragment et les corrections ont été pris en compte dans l'édition dès 1670.

- Unité XIX : une correction p. 307 (Sel. 738), identifiée par M. Pérouse comme étant celle d'Étienne. Ce fragment et sa correction ont été pris en compte dans l'édition dès 1670 .

- Unité XXIII : trois corrections p. $337 \mathrm{v}^{\circ}$ (fragment non retenu dans l'édition - Sel. 473), p. 339 (aprez au lieu de sur, sur étant barré à la sanguine et aprez écrit à la plume - fragment et correction pris en compte dans l'édition dès 1670 -, Sel. 474) et 345 (fragment non retenu dans l'édition - Sel. 489) ${ }^{119}$ identifiées par M. Pérouse comme étant celles d'Étienne.

- Unité XXIV : trois corrections p. 351 vo (Sel. 498) et 353 (Sel. 500) qui pourraient être celles d'Étienne. Le fragment n'a pas été retenu dans l'édition. Il semble que ces interventions sont de la même main qui a écrit les marques en marge (ici un fragment marqué avec des sortes de vagues qui signalent un fragment polémique).

- Unité XXV : trois corrections p. $365 \mathrm{v}^{\circ}$ (Sel. 529 bis), 373 (Sel. 562) et 375 (Sel. 567) identifiées par M. Pérouse comme étant celles d'Étienne. Les trois premières interventions ont été faites sur des fragments ajoutés à l'édition de 1678.

- Unité XXVI : trois corrections p. 387 (Sel. 614), 389 (Sel. 616) et $397 \mathrm{v}^{\circ}$ (Sel. 634) identifiées par M. Pérouse comme étant celles d'Étienne. Les fragments et leurs corrections ont été pris en compte dans l'édition dès 1670.

- Unité XXVIII : trois corrections p. $409 \mathrm{v}^{\circ}$ (Sel. 646) qui pourraient être celles d'Étienne. Une partie du fragment et une correction ont été pris en compte dans l'édition dès 1670.

- Unité XXX : une correction a été portée puis barrée p. 425, sur un texte (Sel. 661) pris en compte dans l'édition dès 1670. L'écriture pourrait être celle d'Étienne.

- Unité XXXIII : une première correction a été portée en marge p. $443 \mathrm{v}^{\circ}$ par Étienne sur une partie de texte (Sel. 426) non retenue dans l'édition. Deux autres interventions ont été faites p. $445 \mathrm{v}^{\circ}$ (Sel. 428) et $453 \mathrm{v}^{\circ}$ (Sel. 437) dans une écriture qui rappelle celle de Nicole sur des fragments non retenus dans l'édition : ces ajouts sont difficiles à décrypter. 


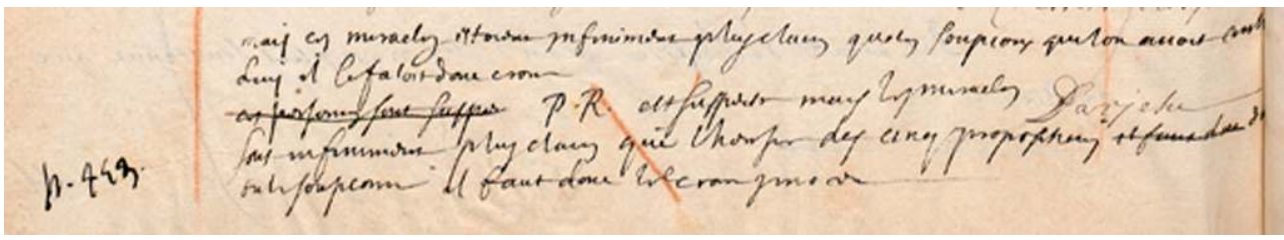

Le texte pourrait être « mais ces miracles estoient infıniment plus clairs que les soupcons que lon avoit cont[re] luy il le faloit donc croire ces personnes sont suspe[ctes] P.R. est suspecté mais les miracles sont infiniment plus clairs que Iheresie des cinq propositions il faut donc d[e] ? ? le soupconn [er] il faut donc le ? ? ?». Il a ensuite été barré à la sanguine et p. 453 a été ajouté dans la marge (voir cette étude à la fin du chapitre).

P. $453 v^{\circ}$

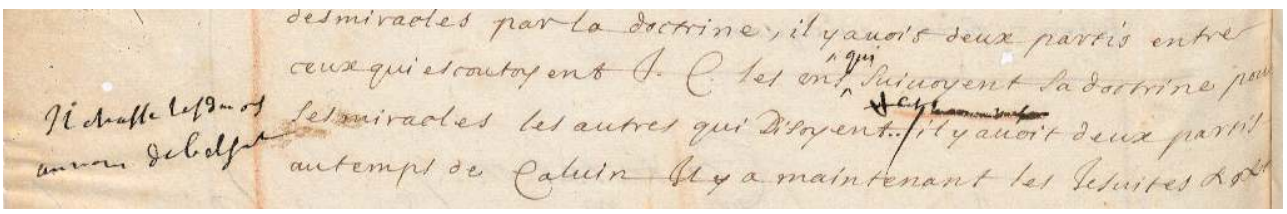

« Il chasse les demons au nom de belz??».

\section{Sélection de nouveaux fragments pour l'édition de 1678 : phase de repérage et corrections}

Vanité: p. $9^{1}($ Sel. $67,68,69,70)$, trois paragraphes ont été barrés et un signe a été ajouté au milieu d'une phrase. Tout ce qui est barré n'a pas été retenu dans l'édition de 1670 ainsi que le texte qui précède ce signe.

Misère : p. 15 (Sel. 91), injustes au lieu de tyranniques (fragment non retenu); p. $15 \mathrm{v}^{\circ}$ (Sel. 91), l'injustice au lieu de la tyrannie (fragment non retenu); p. 17 vº (Sel. 94), le début du texte a été barré jusqu'au mot révérence. Seul ce qui suit immédiatement a été retenu dans l'édition de 1670. Cette intervention est semblable à celle qu'on peut constater dans Misère et Raisons des effets.

Raisons des effets : p. $31 \mathrm{v}^{\circ}$ (Sel. 118), les mots suivants figure et quel mouvement ont été ajoutés dans un texte barré non retenu dans l'édition; p. 37 et $37 \mathrm{v}^{\circ}$ (Sel. 134), un paragraphe a été barré à la sanguine et le reste du fragment est signalé par un trait vertical tracé à la plume dans la marge. Seul le texte non barré a été retenu dans l'édition de 1670.

Philosophes : p. 61, le fragment $n^{\circ} 193$ (Sel. 172) a été barré et n'a pas été retenu dans l'édition.

Souverain bien : p. $65 \mathrm{v}^{\circ}$ (Sel. 181), un signe ovale, suivi de Pyrron. a été écrit dans la marge à la sanguine. Une partie du texte a été intégrée en 1670 dans le chap. XXI relatif aux pyrrhoniens.

Soumission : p. $81 \mathrm{v}^{\circ}$ et 83 , le fragment $\mathrm{n}^{\circ} 233$ (Sel. 210) a été barré et n'a pas été retenu dans l'édition. 
Fausseté des autres religions : p. 105 vo (Sel. 239), un signe <, suivi de Mahomet a été écrit à la sanguine. Une partie du texte a été intégré en 1670 dans le chap. Contre Mahomet.

Preuves de Moïse : p. 153 (Sel. 323), Roys, David establis Isaye Prince du sang a été barré dans un fragment non retenu dans l'édition.

Morale chrétienne : p. 179 (Sel. 396), un signe <, suivi de p. moral a été écrit à la sanguine. Une partie du texte a été intégrée en 1670 dans le chap. Pensées chrétiennes.

Unité I : p. 193 (Sel. 11, 12), un signe < suivi de Marques de la Ver Rel, a été écrit à la sanguine puis barré à l'encre noire. Une partie du texte a été intégrée en 1670 dans le chap. Marques de la véritable religion. Quatre corrections ont été portées par Étienne après les commentaires ajoutés à la sanguine (donc pour l'édition 1678). P. 197 (Sel. 32) : la correction à fond au lieu de à plein est non identifiée ; le fragment n'a pas été retenu dans l'édition.

Unité II : p. $203 \mathrm{v}^{\circ}$ (Sel. 680), une expression a été barrée dans une partie du texte non retenue dans l'édition.

Unité III : p. $213 \mathrm{v}^{\circ}$ (Sel. 681), deux paragraphes ont été barrés et non retenus au sein d'un texte retenu dans l'édition de 1670 ; p. 215 (Sel. 681), seule la phrase non barrée dans un paragraphe a été retenue dans l'édition de 1670 ; p. 217 (Sel. 682), un trait horizontal a été tracé après le deuxième paragraphe. Il signale a posteriori que seuls les paragraphes qui le précèdent ont été pris en compte dans l'édition de $1670 ;$ p. 219 (Sel. 682), un signe < suivi de apres consolateur a été écrit à la sanguine et barré à l'encre noire. Une partie du texte a été intégrée en 1670. Cette instruction signale que le paragraphe est situé après le mot consolateur (fin d'une glose, éd. 1670, p. 8).

Unité IX : p. 245 (Sel. 694), le deuxième paragraphe, non retenu dans l'édition, a été barré. Selon le principe appliqué ci-dessus ce sont les trois premiers paragraphes qui auraient dus être barrés.

Unité XI : p. $253 \mathrm{~V}^{\circ}$, le texte ${ }^{\circ} 56$ (Sel. 702) a été barré et n'a pas été retenu dans l'édition. Dans la marge : un M noir, cerclé de noir et barré d'un X à la sanguine. P. 257 (Sel. 711), la dernière phrase d'un fragment a été barrée : seule cette phrase n'a pas été retenue dans l'édition.

Unité XII, p. $259 \mathrm{v}^{\circ}$, Propheties jusqu'a 267 a été ajouté à la sanguine; p. $269 \mathrm{v}^{\circ}$ (unité XIV) on peut aussi lire Propheties jusqua $279 ;$ p. $277 \mathrm{v}^{\circ}$ (unité XV) : jusqua $283 ;$ p. 287 v $^{\circ}$ (unité XVII) : Propheties jusqua 301. Cela suppose que la pagination a été faite. Le haut de la page qui suit chacune des références est marqué d'un double signe < barré dirigé en partie vers le bas.

Unité XIX, p. 309 (Sel. 738), un signe < suivi de Loy figurative a été écrit à la sanguine. Une partie du texte a été intégrée en 1670 dans le chap. Que la loi était figurative.

Unité XXIII : p. 339 (Sel. 474), un mot a été barré à la sanguine et remplacé par un autre mot ajouté à la plume. P. 341 (Sel. 480), le deuxième paragraphe a été barré et non retenu dans l'édition; dans un autre paragraphe st Augustin a été corrigé $I l$ dans un fragment (Sel. 480) pris en compte dès 1670 mais le début est devenu tout le monde. Un signe en forme de D (qui est plutôt un signe ovale) a été ajouté puis barré à la sanguine.

Unité XXV : p. 383 (Sel. 606), une correction a été proposée sur un fragment signalé comme polémique (vaguelettes) et non retenu dans l'édition. 
Unité XXVI : p. 397 v (Sel. 634), le commentaire et cest la seule raisonnable a été proposé en marge d'un fragment retenu dans l'édition de 1670 mais n'a pas été pris en compte.

Unité XXXIII : p. 439 (Sel. 421), les différentes marques (texte barré, traits verticaux) correspondent aux parties du texte non retenues et retenues dans l'édition de 1670 ; p. $441 \mathrm{v}^{\circ}$ (Sel. 424), le paragraphe, qui précède celui qui a été retenu dans l'édition, a été barré ; p. $445 \mathrm{v}^{\circ}$ (Sel. 428), plusieurs mots ont été barrés dans un texte qui a été retenu dans l'édition de 1670 sans ces termes. Le texte ajouté en bas de la page (par Nicole ?) a été barré en partie à la sanguine (voir ci-dessus) ; p. 449 (Sel. 429), un mot difficile à lire ( di ? ?) a été proposé au-dessus du mot J.-C. (dieu ?) dans un fragment retenu dans l'édition 1670. Ce mot n'a pas été retenu dans l'édition.

Unité XXXIV : p. $455 \mathrm{v}^{\circ}$ (Sel. 439), Une correction a été proposée dans un fragment non retenu dans l'édition ; p. 460 (Sel. 441, 442) et $467 \mathrm{v}^{\circ}$ (Sel. 450), même type de marques qu'à la page 439 (voir ci-dessus) mais aucun texte n'a été retenu dans l'édition ; p. 463 (Sel. 443, 444) et $465 \mathrm{v}^{\circ}$ (Sel. 448), idem, mais les textes signalés correspondent en grande partie à ceux qui ont été retenus ou non dans l'édition.

Lafuma 864 Sel. 439

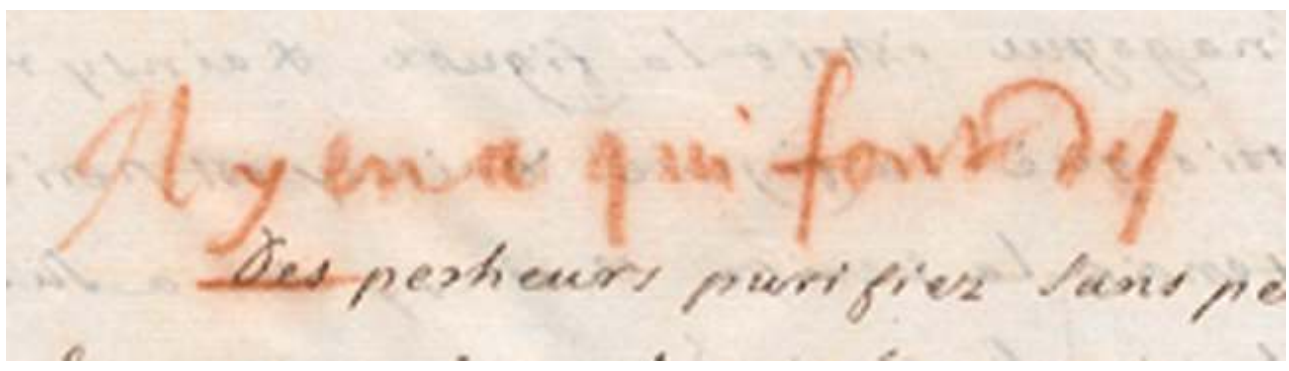

Il y en a qui sont des pécheurs purifiés

\section{Propositions d'intégration}

On ne peut pas vraiment parler de phase car les précisions qui suivent ont pu être notées au cours de la première phase ou juste avant, si ce n'est pendant, la deuxième phase.

- P. 107 v (Fausseté des autres religions, Sel. 245) : un signe < suivi de J.C. a été écrit à la sanguine. Une partie du texte a été intégrée en 1678 dans le chap. J.-C.

- P. $139 \mathrm{v}^{\circ}$ (Loi figurative, Sel. 306) : un signe < suivi de figure a été écrit à la sanguine. Ce texte été intégré dans l'édition 1678 dans le chap. Figures ; un signe <, suivi de moral. a été écrit à la sanguine. Une partie du texte a été intégrée en 1678 dans le chap. Pensées chrétiennes.

- P. 153 (Preuves de Moïse - Sel. 323) : un signe < suivi de foy sans raisonnement a été écrit à la sanguine. Ce texte n'a pas été intégré dans l'édition.

- P. 167 (Prophéties, Sel. 361) : le commentaire pour la fin des preuves de J.C. par les propheties a été ajouté en marge à l'encre noire puis barré. Ce texte n'a pas été intégré dans l'édition.

- P. $177 \mathrm{v}^{\circ}$ (Morale chrétienne, Sel. 391) : un signe < suivi de p. morall. a été écrit à la sanguine. Une partie du texte a été intégrée en 1678 dans le chap. Pensées chrétiennes; un paragraphe (Sel. 392) est précédé d'un trait de séparation suivi du commentaire Commencement des membres pensans, voyez aux 4 pages suivantes; en marge: membre 
pensant ; le tout à l'encre noire ; une partie du texte et plusieurs § qui suivent ont été intégrés en 1678 dans le chap. Pensées morales.

- P. 220 (unité III, Sel. 683) : un signe < suivi de Pensées sur la relig. a été écrit à la sanguine. Un B, mis en valeur dans un cercle (le tout à l'encre noire), a ensuite été ajouté en marge et coché à la sanguine. Le § a été intégré dans l'édition de 1678, chap. Véritable religion prouvée...

- P. 391 v et 393 (unité XXVI - Sel. 619, 620) : un signe < suivi de Object. a été écrit à la sanguine devant deux paragraphes qui n'ont pas été retenus dans l'édition. Un B, mis en valeur dans un cercle et barré en $\mathrm{X}$, a été ajouté dans la marge à la sanguine. Nota : le $\mathrm{B}$ n'a pas la même forme que les précédents et la sanguine est plus rouge que celle de $<$ Object qui est orangée.

- P. 469 (unité XXXIV, Sel. 450) : un commentaire a été ajouté à l'encre noire dans la marge en haut de la page. On pourrait lire Pensées sur les juifs (lecture douteuse).

Les unités Ennui, Grandeur, APR, Religion aimable, Prophéties, Fig. particulières, Conclusion ainsi que VI, VII, VIII, X, XVI, XXX, XXXI, XXXII ne contiennent aucune marque à la sanguine, ni en marge ni dans le texte.

\section{Découverte d'une nouvelle étiquette de liasse}

RO 21-1, Misère $n^{\circ} 18$

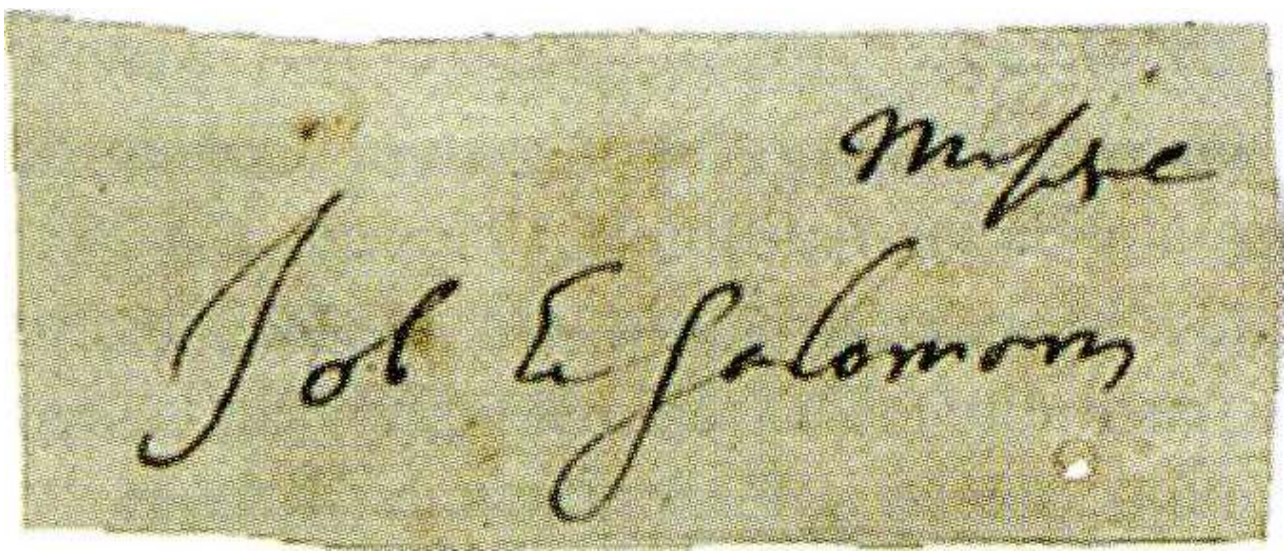




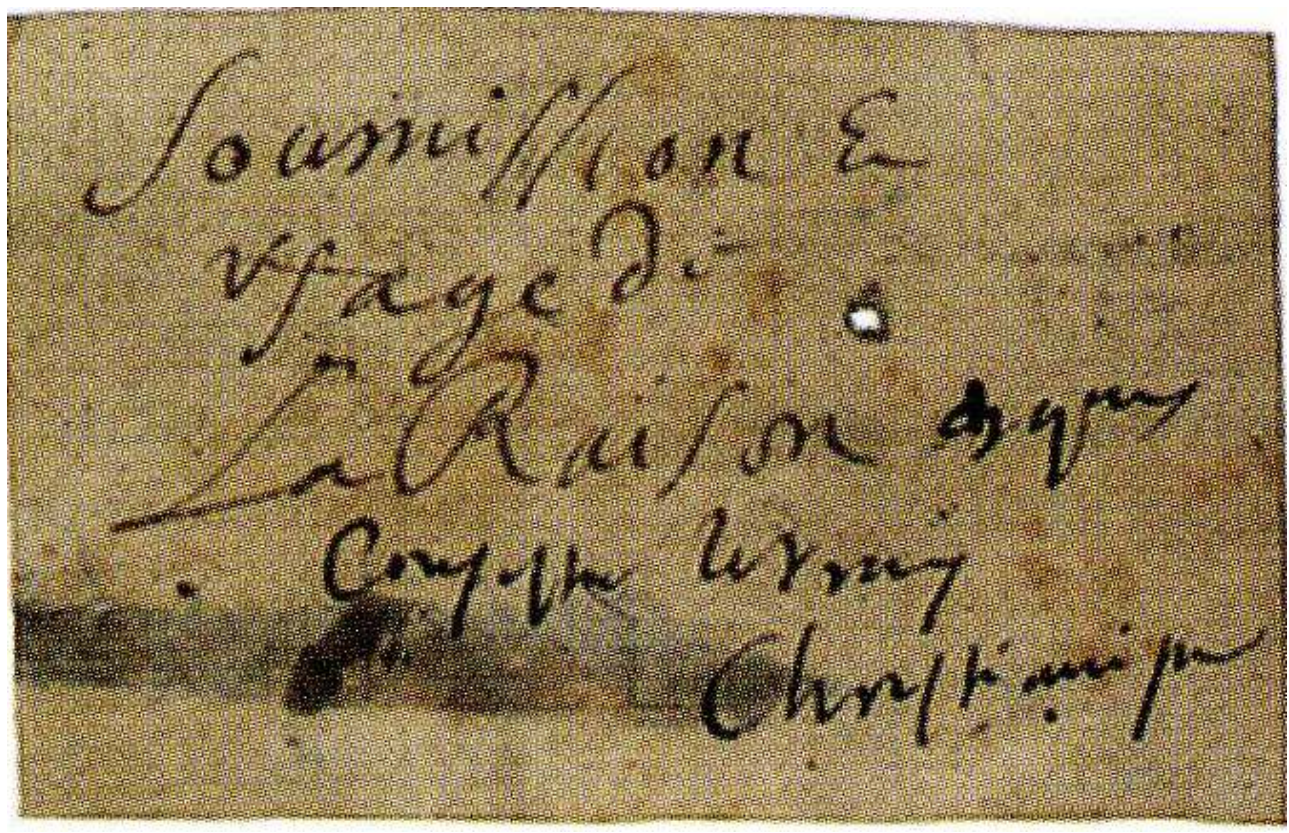

RO 29-2, Loi figurative $n^{\circ} 1$

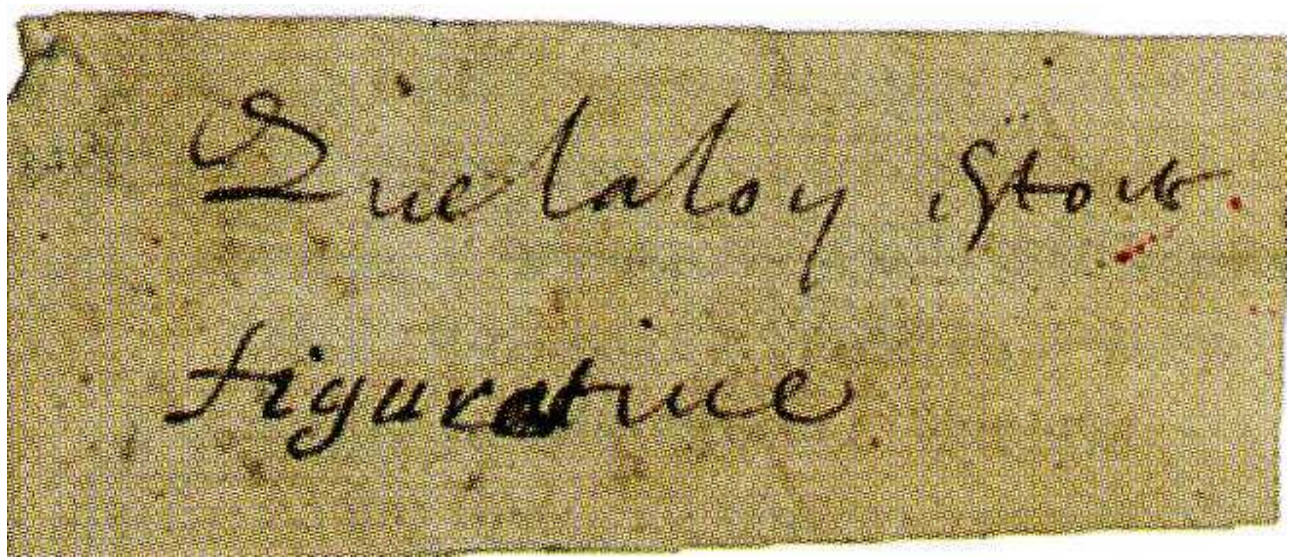

Si les étiquettes, déjà connues, des liasses Soumission et Loi figurative ont été transcrites en tête de leurs liasses respectives, celle de la liasse Misère était passée jusqu'ici inaperçue car elle a été transcrite au milieu de la liasse. Ces trois documents sont les seuls papiers, dans leurs liasses respectives, à porter le titre de la liasse.

\section{NOTES}

1. Centre de Recherches sur les Réformes, l'Humanisme et l'Âge Classique (Université Blaise Pascal, Clermont-Ferrand - UMR 5037, Institut d'histoire de la Pensée classique (C.N.R.S.).

2. Dans la suite de cet article, nous désignons donc par le sigle $R O$ le Recueil des originaux, $C_{1}$ et $C$ ${ }_{2}$ les deux Copies des Pensées, et par le sigle $\mathrm{RC}_{2}$ le recueil qui contient $\mathrm{C}_{2}$, avec d'autres pièces dont la transcription à part de La loi était figurative. 
3. Pensées de M. Pascal sur la religion et sur quelques autres sujets, qui ont esté trouvées après sa mort parmy ses papiers, à Paris chez Guillaume Desprez, 1671, avec Privilège et Approbation. Ce livre est communément appelé édition de Port-Royal.

4. Louis Périer, neveu de Pascal, second fils de Gilberte, avait hérité des papiers originaux à la mort de sa mère en 1687. Sa sœur Marguerite avait hérité des deux Copies. Il était chanoine de la cathédrale de Clermont-Ferrand.

5. Continuation des Mémoires de littérature et d'histoire, t. $\mathrm{V}, 2^{\mathrm{e}}$ partie, Paris, 1728.

6. Pierre Guerrier, cousin de Marguerite Périer et père de l'Oratoire de Clermont depuis 1731, a confectionné plusieurs Recueils (surtout des copies) de documents provenant de la famille de Pascal dont le recueil $\mathrm{RC}_{2}$ et trois recueils (appelés $\mathrm{G}_{1}, \mathrm{G}_{2}, \mathrm{G}_{3}$ par J. Mesnard) dont le ms 13913 fonds français $\left(G_{3}\right)$.

7. D’où il extrait notamment le texte qui était inédit «Il faut dire en gros : «Cela se fait par figure et mouvement ", car cela est vrai. Mais de dire quelle figure et mouvement et composer la machine, cela est ridicule, car cela est inutile et incertain et pénible. Et quand cela serait vrai, nous n'estimons pas que toute la philosophie vaille une heure de peine. » (Sel. 118).

8. D'où il extrait deux textes alors inédits (voir la section consacrée aux marques dans $C_{2}$ ).

9. Notice sur les familles Pascal et Périer, p. 120 : «M. Guerrier de Bezance, aujourd'hui Maître des Requêtes, appartient à la famille de Pascal, du côté maternel. Notre édition doit à son amour pour les Lettres \& à son respect pour la mémoire de Pascal, plusieurs Ouvrages de ce grand Homme qui n'avoient pas encore été imprimés ».

10. Augustin Renouard (1812) : «Comme l'écriture de Pascal, fort pénible à lire, étoit plus indéchiffrable encore sur ces brouillons écrits à la hâte et pour son seul usage, on eut aussi la précaution d'en faire une copie exacte ; mais on ne s'y astreignit point à l'ordre suivi dans l'édition de 1670 , et ses nombreuses réimpressions. [...]»

11. Édition paléographique des manuscrits originaux conservés à la Bibliothèque Nationale enrichie de nombreuses leçons inédites et présentée dans le classement primitif avec une introduction et des notes descriptives, Paris, Librairie philosophique J. Vrin, 1942, Introduction p. 13.

12. Blaise Pascal, Discours de la condition de l'homme, Paris, éd. Albin Michel, 1948, Introduction p. 8 .

13. "Aux origines de l'édition des Pensées: les deux copies", in Les "Pensées de Pascal ont trois cents ans, Clermont-Ferrand, G. de Bussac, 1971, p. 1-30.

14. Lorsqu'un copiste reproduit un document, il est obligé de diriger son regard tantôt sur l'original tantôt sur sa copie. Si une séquence, ou un mot caractéristique, existe deux fois dans un même texte, séparée par une ligne ou quelques mots, le regard va parfois se poser sur la deuxième occurrence et le copiste va sauter une partie du texte. Ce phénomène est caractéristique des copies.

15. Pascal : Pensées, présentation et notes par Gérard Ferreyrolles, texte établi par Philippe Sellier d'après la copie de référence de Gilberte Pascal, Librairie générale française, Coll. Classiques de poche, 2000, Introduction p. 20.

16. Blaise Pascal, Pensées. Opuscules et lettres, édition de Philippe Sellier, Paris, Éditions classiques Garnier, 2010.

17. Voir le tableau détaillé en Annexe, p. 44.

18. Ph. Sellier définit aussi 4 parties A, B, C, D : A. Le projet de juin 1658 ; B. Les dossiers mis à part en juin 1658 ; C. Les derniers dossiers de pensées mêlées (juillet 1658-juillet 1662) ; D. Les développements de juillet 1658 à juillet 1662.

19. Ce titre est uniquement dans $C_{2}$.

20. Voir Dominique Descotes et Gilles Proust, « Un projet du Centre international Blaise Pascal : l'édition électronique des Pensées» in Courrier du CIBP n³0, Clermont-Ferrand, 2008, "III. Remarques sur l'ordre des Copies ", p. 12.

21. Ce qui fait qu'on peut savoir si le trou a été fait à partir du recto ou du verso du papier. 
22. Au moment où nous écrivons cet article nous n'avons pas encore terminé cette observation minutieuse. Il est donc possible que deux ou trois trous aient été oubliés.

23. Le nombre de papiers perdus ne tient pas compte des titres d'unités dont seules deux étiquettes ont été conservées dans le RO. D'autre part on ne peut pas savoir si deux textes consécutifs étaient écrits sur deux papiers perdus ou sur un seul.

24. Seuls les trous susceptibles de correspondre à un enfilage sont comptabilisés. Certains papiers ont été traversés par un ver après leur collage dans le Recueil.

25. Il est possible que les papiers 265-7 (troué) et 265-8 (non troué) n'en faisaient qu'un (Laf. 190) et qu'ils ont été séparés avant d'être collés dans le Recueil.

26. Il est possible que les papiers 467-8 (troué) et 457-3 (non troué) n'en faisaient qu'un (Laf. 209) et qu'ils ont été séparés avant d'être collés dans le Recueil.

27. Il s'agit du papier collé dans le Recueil des originaux p.365. Ce feuillet provient de la partie droite d'une feuille marquée d'un double filigrane Grappe de raisin (un filigrane par feuillet). Le feuillet qui provient de la partie gauche de cette feuille a été rogné avant d'être collé sur la page 70 , notamment en bas à droite où aurait pu se situer un éventuel trou d'enfilage. Qu'ils aient été séparés ou non par Pascal, dans les deux cas le feuillet de la page 365 aurait dû être troué si Pascal les avaient réunis dans une liasse.

28. Est-ce que les unités III et IV n'en formaient qu'une? Jean Mesnard, Les Pensées de Pascal, Paris, SEDES, 1976, p. 25, affirme que l'unité III était un «cahier, à l'intérieur duquel s'était trouvée rangée l'unité IV ».

29. Ph. Sellier suggère deux fils parallèles. Nous pensons que les trous auraient été déformés, ce qui n'est pas le cas.

30. Comment appeler une unité constituée d'un seul papier troué ? Une liasse ? Un dossier ? Une feuille volante?

31. "Les Juifs charnels tiennent le milieu entre les chrétiens et les païens. Les païens ne connaissent point Dieu et n'aiment que la terre, les Juifs connaissent le vrai Dieu et n'aiment que la terre, les chrétiens connaissent le vrai Dieu et n'aiment point la terre. Les Juifs et les païens aiment les mêmes biens, les Juifs et les chrétiens connaissent le même Dieu.

Les Juifs étaient de deux sortes : les uns n'avaient que les affections païennes, les autres avaient les affections chrétiennes. »

32. "Les Juifs charnels et les païens ont des misères et les chrétiens aussi. Il n'y a point de rédempteur pour les païens, car ils n'en espèrent pas seulement. Il n'y a point de rédempteur pour les Juifs, ils l'espèrent en vain. Il n'y a de rédempteur que pour les chrétiens. »

33. Ce papier a été monté sur onglet à sa demande le 6 novembre 1935.

34. Cela nous paraît possible, mais le mot «Aymable » ne semble pas autographe, contrairement au renvoi "voyez Perpetuité ", et si le premier copiste a jugé utile de transcrire le renvoi à Perpétuité ( $C_{1 \mathrm{p}}$. 113, $C_{2}$ p. 139), l'autre renvoi n'a pas été transcrit. Doit-on pour autant en déduire qu'il a été ajouté post mortem?

35. Selon $\mathrm{M}^{\text {me }}$ Tandeau de Marsac, Présidente de Connaissance et Sauvegarde de Saint-Léonard, que je remercie ici cordialement, la famille Mabaret (ou Masbaret) la Rue fut propriétaire du moulin de Chez le Pénitent sur la Vienne jusqu'au XIX ${ }^{\mathrm{e}}$ s. et sans doute bien avant 1742, date à laquelle $\mathrm{M}$ elle de La Chassagne, veuve Mabaret, a donné à bail le moulin. $\mathrm{M}^{\text {me }}$ Tandeau de Marsac précise que les éditeurs parisiens qui achetaient le papier limousin achetaient le papier à un négociant qui mélangeait tous les papiers. Donc ce n'est pas parce qu'un papier provient du Limousin, de Normandie ou d'Auvergne qu'il faut en déduire que la reliure a été faite en province.

36. La première page de garde du Recueil des originaux porte le filigrane MOYNE./NORMANDIE/ MVLENTAIGNE/16 (sur quatre lignes) qui pourrait correspondre à une marchande papetière de la vallée des Vaux, paroisse de St Germain de Tallevande (14), Marie Lentaigne (1688-1757) veuve de Julien Laisné, sieur des Moulins. MOYNE pourrait signifier soit papier MOYEN, le E et le N étant 
inversés, soit Le Moyne, nom des seigneurs de Sourdeval (vallée de la Sée). Le dernier feuillet, à la fin du Recueil, porte en filigrane un grand raisin muni d'une longue queue.

37. Jean Guerrier était un cousin de Marguerite Périer et Pierre Guerrier était un neveu de Jean. Selon L. Lafuma (1959), cette Copie a été donnée en 1715 à dom Jean Guerrier, prieur de l'abbaye de Saint-Jean-d'Angély, par Marguerite Périer nièce de Pascal. Il s'appuie sur le commentaire de Dom Tassin, Histoire littéraire de la congrégation de Saint-Maur, 1770, p. 786: "Ayant engagé Mademoiselle Perrier [sic], nièce du célèbre $\mathrm{M}$. Pascal, à lui céder ceux [les livres] de la bibliothèque de son oncle, il les fit transporter à Saint-Jean-d'Angély; mais il envoya les manuscrits au Père Général à Paris. Il se réserva la manuscrit des Pensées de $\mathrm{M}$. Pascal, parce qu'il en contenait qui n'avaient pas pu être imprimées. Il envoya ce recueil à Dom Alaydon Général de la Congrégation quelques mois avant sa mort. Afin de ne rien perdre de ce qui venait de la bibliothèque de Mademoiselle Perrier, Dom Guerrier fit faire des recueils d'une infinité de brochures et de feuilles volantes, et autres écrits qui se trouvaient parmi les livres. Il les fit relier proprement, [...]." Selon J. Mesnard, OC I, p. 113, ce texte a peut-être été écrit peu de temps après que Marguerite Périer lui en a fait don (qui daterait donc de 1723 et non de 1715); le don à l'abbaye bénédictine de Saint6germain-des-Prés a pu être effectif plusieurs années après. Selon Dom Tassin, Ibid., p. 788, Jean Guerrier serait décédé le 31 octobre 1731.

38. Même encre et même écriture que la numérotation des paragraphes.

39. Les marques correspondaient souvent aux armes des clients prestigieux des marchands papetiers (imprimerie royale, contrôleur général des finances, archevêque, etc.)

40. Les feuilles utilisées dans le cahier Perpétuité sont plus petites : $43 \mathrm{~cm} \times 34 \mathrm{~cm}$.

41. R. Gaudriault, Filigranes et autres caractéristiques des papiers fabriqués en France aux XVII et XVIII ${ }^{e}$ siècles, C.N.R.S. Éditions - Éditions J. Telford, Paris, 1995. La planche 56, nº 433, montre un filigrane similaire portant les initiales CB et datant de 1649 .

42. Le Gaudriault montre deux filigranes de ce type dans les armoiries de France et Navarre (planche 17, $\mathrm{n}^{\circ} 119$ (daté de 1648) et 120 (vers 1650).

43. Env. 1660 selon Gaudriault, pl. 148, n 4250.

44. Sa position n'est pas due au hasard. Le texte est en relation étroite avec l'avant dernier fragment de la liasse Preuves de Moïse : «Sem qui a veu Lamech, qui a veu Adam a veu aussy Jacob ; Qui a veu ceux qui ont veu Moyse, donc le deluge \& la creation sont vrais, cela conclud entre de certaines gens qui l'entendent bien.» (Sel. 327). M. Le Guern édite le fragment autographe juste après ce texte.

${ }^{45}$ Voir la description détaillée de ce Recueil dans J. Mesnard, OC I, p. 294-298.

45. ${ }^{46} \mathrm{La}$ comparaison de l'écriture avec les documents proposés comme des exemples d'autographes de Pascal dans le RO est négative. On peut constater plusieurs abréviations qu'on ne retrouve pas dans les papiers de Pascal : generaons pour générations, $q$ pour que, comcoit pour commençait, seulem ${ }^{t}$. pour seulement, veneraon pour vénération (nous soulignons les lettres surmontées d'un arc de cercle dans le manuscrit).

46.

47. Pierre Guerrier a aussi recopié un texte en bas de la page $467 \mathrm{v}^{\circ}$, laissée vierge par le copiste et située au verso de l'unité X. Ce texte est absent de $C_{1}$ et porte le $n^{\circ} 698$ dans l'éd. Sellier. Aucun papier original ne correspond à ce texte. Selon J. Mesnard, Textes inédits de Blaise Pascal, 1962, p. 25, Pierre Guerrier aurait recopié un autographe de Pascal placé ici pour orner la Copie comme le fait le feuillet autographe (toujours selon J. Mesnard) dans $C_{1 \text { p. }}$ 154. Ce qui est sûr c'est que la copie Sainte-Beuve (copie du manuscrit Périer) et la copie Montempuys (voir J. Mesnard, OC I, p. 307), dont la source est aussi le ms Périer, reproduisent au moins une partie de ce texte. Le ms Périer a été établi avant 1711 (décès de Louis Périer) en grande partie à partir de la Copie $C_{2}$ et Pierre Guerrier n'a travaillé sur $C_{2}$ qu'à partir de 1731 . Le texte était donc bien dans $C_{2}$ avant que Pierre Guerrier ne le recopie. 
48. Ph. Sellier pensait que ce titre était marqué d'un signe de type astérisque. Il s'agit en fait d'une rousseur dans le papier.

49. On ne connaît pas le nom de ce copiste. Nous ne disposons d'aucun document portant une telle écriture. Dans la notice sur le duc de Roannez (p. 877) du Dictionnaire de Port-Royal (élaboré sous la direction de Jean Lesaulnier et Antony McKenna, Paris, Champion, 2004), A. McKenna dit qu' « il est possible que les deux célèbres copies des Pensées aient été établies par le secrétaire du duc de Roannez, Nicolas Martine. ".

50. On relève quelques exceptions dans $C_{1}: p .221 v^{\circ}$ (unité IV), le copiste avait annoncé le cahier suivant (unité V), la réclame a été barrée par le réviseur ; p. 421 la réclame a été oubliée ; et dans $\mathrm{C}_{2}: 1 /$ ajoutées en fin d'unités: p. 32 : réclame Misere; p. 74 : réclame Divertissement; p. 253 (dossier XXXIII) : réclame Injustes, premier mot du dossier XXXIV; p. 418 (dossier II) : réclame Qu'ils, premier mot du dossier III ; 2/ absence de réclame: p. 241, 287, ainsi qu'au recto des feuillets non écrits au verso : p. 347, 349, 357, 359, 361, 363, 365, 367, 369, 371).

51. On trouve aussi le signe ./. à la fin des paragraphes sur des papiers des Pensées, écrits de la main de Gilberte Périer, ainsi qu'à la fin de chaque ligne de l'attestation de la copie du Mémorial par Louis Périer dans le RO.

Dans $C_{1}$, seules les p. 173 (Figures particulières), 237 (unité VII) et 249 (unité X), n'en ont pas, les dossiers étant copiés sur une seule page. De même la p. 437 (fin de l'unité XXXII).

52. Voir $C_{1}$, pages $23,113,245 \mathrm{v}^{\circ}$. Dans $C_{2}$, il n'y a pas de telles marques p. 102, entre APR et Commencement ; p. 111, entre Soumission et Excellence; p. 215, entre Morale chrétienne et Conclusion; p. 224, entre les dossiers XXXV et le titre Miracles; p. 225, après le titre Miracles; p. 409 après le titre Preuves de la religion..., et p. 433, entre les dossiers III et IV.

53. Exceptions dans $C_{1}$ (paragraphes sans alinéa) : voir p. 79, Commencement ajouté à gauche de Cachot; les $P$. ajoutés à gauche des $§$ p. 83 ; les numéros des Preuves p. 137vº-139; Dit ainsi., p. 263 ; La sincérité des juifs..., p. $301 \mathrm{v}^{\circ}$; Objec. et Resp. p. 393 et 395. Exceptions dans $\mathrm{C}_{2}$ (paragraphes sans alinéa) : très souvent, le copiste présente les paragraphes sans aucun retrait lorsque le texte est très court (moins d'une ligne). Exemples : p. 13, 85, 99, 103, etc.

54. Exemples dans $C_{1 \text { p. }} 353$ et $357 v^{\circ}$. En revanche, le trait qui a été ajouté p. 217 (Unité III - le dossier est perdu) n'a pas pour objet de signaler un nouveau papier. Ce trait a été fait à la sanguine par Étienne pour délimiter le texte qui a été retenu dans l'édition (voir l'étude des marques à la sanguine utilisées pour repérer des textes intégrés dans l'édition).

55. Voir dans $C_{1 \text { p. }} 225,291 v^{\circ}$ (sous réserve), 393, 395, 425, et en particulier (dossiers XXXIII et XXXIV) : p. 441, $441 \mathrm{v}^{\circ}, 443 \mathrm{v}^{\circ}, 445,447 \mathrm{v}^{\circ}, 449,449 \mathrm{v}^{\circ}, 451,453,455 \mathrm{v}^{\circ}, 457,457 \mathrm{v}^{\circ}, 460 \mathrm{v}^{\circ}, 463$, $465,467,467 \mathrm{v}^{\circ}$; et dans $\mathrm{C}_{2}$, pages 86, 87, 115, 209, 211 à 215, 221, 242, 243, 247, 256, 258, 264, 266, 267, 277, 290, 347, 353, 401 (copie Guerrier), 438. La présence d'un trait p. 438 (unité V) notamment est intéressante car les papiers ont disparu.

56. Voir dans $C_{1 \mathrm{p} .} 13 \mathrm{v}^{\circ}$ (fin du $2^{\text {ème }}$ cahier atteinte trop rapidement), $35 \mathrm{v}^{\circ}, 55 \mathrm{v}^{\circ}, 77 \mathrm{v}^{\circ}, 79,81,107 \mathrm{v}$ $\circ, 365,371,381,381 \mathrm{v}^{\circ}, 427 \mathrm{v}^{\circ}$. Voir dans $\mathrm{C}_{2}$, pages $2,4,5,7,8,9,10,15,32,33,41,103,104,152,179$, $325,325 \mathrm{v}^{\circ}, 327 \mathrm{v}^{\circ}, 329$.

57. Dans $C_{1}$, les textes écrits entièrement en latin ne sont pas soulignés.

58. Voir p. $13 \mathrm{v}^{\circ}$.

59. Exceptions : le titre est souligné p. $141 \mathrm{v}^{\circ}$ (Du péché originel, tradition ample du péché originel selon les juifs) et $197 \mathrm{v}^{\circ}$ (La nature est corrompue) ; le titre n'est pas centré p. 77 et 253.

60. Exceptions (titres non soulignés) : p. 34 et 489.

61. Ce qui montre bien que $C_{1}$ n'a pas été copiée à partir de $C_{2}$ et réciproquement. Ces cas montrent à eux seuls la nécessité d'une troisième Copie.

62. Cas significatifs non corrigés par le réviseur dans $C_{1}$ : oubli de et puis montrer qu'elle est vraie p. 3 ; oubli de parce qu'elles sont lois comme il faut obéir p. $19 \mathrm{v}^{\circ}$ (saut du même au même); oubli de l'opinion qui détruisait p. 35 ; oubli de misérable p. $119 \mathrm{v}^{\circ}$; oubli de il faut donc par nécessité qu'ils ne soient que figures, on ne saurait pas même accorder les passages p. $127 \mathrm{v}^{\circ}$ (saut du même au même); 
oubli de et son règne spirituel p. $129 \mathrm{v}^{\circ}$; l'aprouvent au lieu de la prouvent p. $201 \mathrm{v}^{\circ}$; cette au lieu de notre p. 229 ; oubli de et Rome admise : Dixit dominus. Que le nom des juifs serait réprouvé p. 243 (saut du même au même) ; historiens au lieu de histoire p. 257 ; les au lieu de vos p. 261 vo ; Ame au lieu de Art p. 373 ; en au lieu de entre p. $373 \mathrm{v}^{\circ}$; elles au lieu de ils p. 385, comme substantée au lieu de transsubstantiée p. 387 ; loix au lieu de voies p. 397 v ; rédempteur au lieu de réparateur p. 409 ; oubli de on se gâte l'esprit et le sentiment par les conversations p. 421 (saut du même au même).

Cas significatifs non corrigés par le réviseur dans $C_{2}$ : un fait au lieu de un effet p. 1 ; avec au lieu de contre p. 36 ; c'est à dire au lieu de c'est dire p. 47 ; pour au lieu de tout p. 69 ; intelligible au lieu de inintelligible p. 73 ; misérable au lieu de malheureuse p. 77 ; par les au lieu de parlent p. 127 ; qui a t'il au lieu de qu'y a t'il p. 146 ; § non barré p. 218 ; par au lieu de pour p. 242 ; parties au lieu de partis p. 253 ; preuves ils au lieu de preuve s'ils p. $257 \mathrm{v}^{\circ}$; faict au lieu de faut p. 275 ; oubli de et cependant les philosophes ont conduit la morale p. $311 \mathrm{v}^{\circ}$ (saut du même au même); sujets au lieu de suspects p. $321 \mathrm{v}^{\circ}$; par au lieu de pour p. $323 \mathrm{v}^{\circ}$; plus tost au lieu de plus p. $331 \mathrm{v}^{\circ}$; à au lieu de en p. $339 \mathrm{v}^{\circ}$; séparé au lieu de s'étant p. 363 ; serment au lieu de sermon p. 371 ; en disant in communi au lieu de en disant de in communi p. 383 ; qui au lieu de qu'il p. 451 ; qu'il au lieu de qui p. $485 \mathrm{v}^{\circ}$; miserabilia au lieu de mirabilia p. 503 ; voila au lieu de voilez p. 511.

63. Le nombre important d'hésitations du copiste et de fautes corrigées par le réviseur dans l'unité II (Argument du pari) a fait penser à J. Mesnard (Ibid, 1971, p. 15) que $C_{1}$ a été réalisée en parallèle avec $C_{0}$. Cela peut aussi s'expliquer par l'état plus ou moins chaotique de la transcription dans $C_{0}$ lié aux nombre de textes barrés par Pascal et au niveau de difficulté de compréhension du texte, ce qui d'ailleurs a justifié la réalisation de nouvelles copies plus nettes.

64. $Y$ compris dans $C_{2}$ qu'on ne peut déclasser car la plupart des textes sont écrits à cheval sur au moins deux cahiers.

65. Seule la comparaison des Copies avec le manuscrit original permet de repérer les textes barrés par Pascal et qui ont été transcrits. Dans tous les cas ces textes sont nécessaires à la compréhension de la phrase ou du fragment. Textes barrés : $C_{1 \text { p. }} 15\left(C_{2 \text { p. }} 33-\right.$ Sel. 88); $C_{1 \text { p. }} 17\left(C_{2 p}\right.$. 36 - Sel. 94); $C_{1 \text { p. }} 173\left(C_{2 \text { p. }} 207\right.$ - Sel. 382); $C_{1 \text { p. }} 267\left(C_{2 \text { p. }} 485\right.$ - Sel. 719); $C_{1 \text { p. }} 335$ vº $\left(C_{2 \text { p. }}\right.$ 289, barré par le réviseur - Sel. 467); $C_{1 \text { p. }} 355 \mathrm{v}^{\circ}\left(C_{2 \text { p. }} 313\right.$ - Sel. 507) ; $C_{1 \text { p. }} 425 \mathrm{v}^{\circ}$ ajouté par le réviseur (n'est pas dans $C_{2 \text { p. }}$ 399, copie de P. Guerrier - Sel. 663).

66. Ph. Sellier (Ibid., 2010, Introduction p. 33) suppose qu'un papillon de liasse intitulé Opinions du peuple saines (barré) avait été conservé au-dessus de la liasse Raisons des effets.

67. Nous rappelons que le titre a été souligné par le copiste dans $C_{1}$ et qu'il existe un autre cas de titre souligné p. $141 \mathrm{v}^{\circ}$. Ce titre est aussi souligné dans $C_{2}$ (p. 10) mais tous les titres le sont et rien n'a été ajouté pour le distinguer des autres.

68. Ce thème apparaît aussi dans un des plans envisagé par Pascal (Sel. 40). Le texte de Sel. 35 est très proche de Sel. 40. Il est aussi question de ce thème dans Sel. 529 bis (Pensées diverses).

69. Voir Dominique Descotes et Gilles Proust, Ibid., 2008, « II. Découverte de deux textes disparus $\mathrm{du}$ Recueil des Pensées et hypothèse sur la disparition de deux autres textes », p. 7.

70. Le titre (ou sous-titre) «Aveugler» apparaît aussi un peu plus bas au verso du papier. Seul le titre Aveugler, éclaircir a été transcrit dans les Copies $\left(C_{1 \text { p. }} 119, C_{2 \text { p. }}\right.$ 145).

71. J. Mesnard, Ibid, 1971, est persuadé que la table des matières proposée dans les Copies est une copie figurée d'un autographe perdu et que tous les titres sont de Pascal (et non pas de Nicole et Arnauld, comme le suggérait L. Lafuma (1949), ou du premier copiste). J. Mesnard précise que cette table représente un classement et non pas un plan.

72. Philippe Sellier, "Indications d'ordre et dossiers pascaliens ", in "Les Pensées de Pascal: interprètes et éditeurs en débat ", Revue des sciences philosophiques et théologiques, CNRS, $\mathrm{n}^{\circ} 1$, janvier-mars 2009, p. 145. Ph. Sellier appelle cette table un récapitulatif de l'état des dossiers.

73. L'apologétique de Pascal, Paris, P.U.F, 1958, J. Mesnard (1971) conteste cette objection en posant la question «Comment cette affirmation serait-elle conciliable avec le fait d'une série de chapitres portant des titres et ordonnées les uns par rapport aux autres?». 
74. Voir notamment Laurent Thirouin, «Les premières liasses des Pensées: architecture et signification ", XVII siècle, $\mathrm{n}^{\circ}$ 177, 1992.

75. Il est curieux de constater que ce diptyque correspond au dernier papier de la première liasse (Ordre - Sel. 46) qui pourrait être le premier papier enfilé.

76. L'analyste informaticien vous dirait que ce type d'organisation rappelle celui des bases de données relationnelles, dont la conception s'adapte très bien à une utilisation souple et multiple des données.

77. Nous adoptons la distinction faite par J. Mesnard : les Copies ont été revues et corrigées par un réviseur puis des corrections ont été proposées par des correcteurs pour l'édition.

78. Ou à la mine de plomb (graphite).

79. On peut observer de nombreuses corrections de la ponctuation p. 1, 2, 3, etc., de nombreux ajouts p. 209, 245, 247, etc. ainsi que des suppressions p. 5 et 8.

80. Il s'agit des cahiers Ordre, Vanité, Ennui, Raison des effets, Contrariétés, Divertissement, Philosophes et Figures particulières.

81. En particulier «Toutes choses leur arrivaient en figures» (texte original) est transcrit figure dans cette copie et $\mathrm{C}_{1}$ et figures dans $\mathrm{C}_{2}$. " qui n'ont de déplaisir que d'être privés de sa vue » (texte original) est transcrit déplaisir dans cette copie et $C_{1}$ et déplaisirs dans $C_{2}$. En revanche, le copiste ne fait pas l'erreur du saut du même au même présente dans $C_{1}$ : «Il faut donc par nécessité qu'ils ne soient que figures. On ne saurait pas même accorder les passages ». "Il faut voir si les Prophètes en parlant de ces choses y arrêtaient leur vue et leur pensée en sorte qu'ils n'y vissent que cette ancienne alliance " (texte original) est transcrit n'y dans cette copie et $C_{2}$ et ne dans $C_{1}$. «sa chair et ce qui en sortait que pour cela il les avoit multipliés et distingués de tous les autres peuples sans souffrir qu'ils s'y mêlassent " (texte original) est transcrit sortait dans cette copie et $\mathrm{C}_{2}$ et sortirait dans $\mathrm{C}_{1}$. «Les Rabbins prennent pour figure les mamelles de l'épouse " (texte original) est transcrit figure dans cette copie et $\mathrm{C}_{2}$ et figures dans $\mathrm{C}_{1}$. "dans une terre grasse » (texte original) est transcrit une dans cette copie et $\mathrm{C}_{2}$ et la dans $\mathrm{C}_{1}$.

82. Il s'agit de la Maxime $n^{\circ}$ LXXXI qui est aussi la dernière maxime publiée; le texte est légèrement différent de l'original.

83. Il n'est pas sûr que ce soit le réviseur.

84. Ph. Sellier (Ibid, 2010, Introduction p. 34 note 1) dit que le titre Preuves de la religion par le peuple juif, les prophéties et quelques discours n'est vraisemblablement pas de Pascal.

85. Ce papier est coté 2 . La première page du texte $A P R(R O$ p. 317) est cotée 1 (visible en transparence du verso).

86. On notera au passage que les liasses titrées étaient dénommées Chapitres dans $C_{0}$.

87. Cette hypothèse exclut une omission simultanée dans les deux Copies corrigée ou non dans les deux Copies par les réviseurs.

88. On peut en effet lire raisons.

89. Pascal écrit souvent les $g$ comme des $q$.

90. C'est aussi ce qu'avait constaté Prosper Faugère en 1844. Faugère a écrit d'ailleurs la note suivante sur l'exemplaire de la Bibliothèque Mazarine (selon Maire, $\mathrm{n}^{\circ} 10$ p. 108) : "Cette édition est non seulement tout à fait conforme pour le texte avec la $2^{\mathrm{e}}$ édition de 1670 , mais les ornements et les caractères sont identiques; les mêmes fautes typographiques se rencontrent dans l'une et dans l'autre ; enfin il est évident que la même composition a servi pour les deux éditions. Tout le changement consiste à avoir substitué dans le titre le mot troisième au mot deuxième. "

91. La modernisation de -aym- en -aim- a été faite dès la deuxième édition. Mais le copiste de $C_{1}$ et $C_{2}$ utilise partout la graphie verbale -aym- à une exception près.

92. Voir L. Lafuma, Pascal. Pensées sur la religion. Documents, Paris, Éd. du Luxembourg, 1951, p. 83. 
93. J. Mesnard (1971, p. 18) : « l'écriture d'Arnauld et de Nicole ne figurent jamais ensemble dans un même cahier : manifestement, une distribution a été effectuée, limitée d'ailleurs aux cahiers les plus importants. »

94. Si le nom des correcteurs semble établi, les avis divergent quant à l'auteur de certaines interventions, en particulier celles d'Étienne et de Nicole. P.-L. Couchoud voyait dans les marques ajoutées en marge pour l'édition de 1678 l'écriture d'Arnauld; L. Lafuma pensait aussi que les marques à la sanguine étaient d'Arnauld; voir aussi Marie Pérouse, L'invention des Pensées de Pascal. Les éditions de Port-Royal (1670-1678), Paris, éd. Champion, 2009, p. 33-43.

95. Nombre de fragments concernés.

96. Les copies conservées dans les Portefeuilles Vallant (BNF $m s$ fr. 17.049) sont les seuls témoins, malheureusement limités à quelques chapitres, du travail effectué par le Comité. Voir M. Pérouse, Ibid., p. 43-47 et Annexe III.

97. Contrairement à P.-L. Couchoud et L. Lafuma qui reconnaissaient l'écriture d'Arnauld. Il est vrai qu'il est difficile de comparer une écriture à la sanguine avec la finesse d'une plume.

98. En agrandissant la photo, on peut voir très nettement que le signe < est sous le cerc le noir et non l'inverse.

99. Ce texte a servi de base à un développement ajouté p. 28 à 33 de l'édition.

100. Des petites phrases ont aussi été ajoutées, p. $71(\S 2)$ - qui provient de $C_{1 \text { p. }} 301 \mathrm{v}^{\circ}$ (XVIII) -, et p. 332 (§ 33) - qui provient de $C_{1 \text { p. }} 317$ (XXI).

101. «Nous voyons aussi sur cette Copie les annotations, à la sanguine ou à l'encre, des censeurs parisiens : la lourde main d'Arnauld qui marque d'un B (Bon) ou d'un M (mauvais) des morceaux à retenir ou à écarter [...] ".

102. Voir l'illustration de la page 81 au début de cet article.

103. Nous passons sous silence les rares exceptions et erreurs inhérentes à ce type de travail. Cette concordance, très fiable, a été réalisée avec beaucoup de soin. Aucune correction de texte ne semble avoir été notée sur la Copie durant ce travail.

104. Il s'agit de Jean Guerrier, bénédictin de la congrégation de Saint-Maur et non de Pierre Guerrier, oratorien.

105. Dom Tassin, Histoire littéraire de la congrégation de Saint-Maur, 1770, p. 786 : "Ayant engagé Mademoiselle Perrier, niece du célebre M. Pascal, à lui céder ceux [les livres] de la bibliotheque de son oncle, il les fit transporter à Saint-Jean d'Angély; mais il envoya les manuscrits au Pere Général à Paris. Il se réserva le manuscrit des Pensées de $\mathrm{M}$. Pascal, parce qu'il en contenoit qui n'avoient pas pu être imprimées. Il envoya ce recueil à Dom Alaydon Général de la Congrégation quelques mois avant sa mort. Afin de ne rien perdre de ce qui venoit de la bibliotheque de Mademoiselle Perrier, Dom Guerrier fit faire des recueils d'une infinité de brochures \& de feuilles volantes, \& autres écrits qui se trouvoient parmi les livres. Il les fit relier proprement, [...]. ».

106. Dans la transcription des dossiers non conservés dans le $R O$ les « 8 » ont été ajoutés uniquement devant les paragraphes numérotés. L'inverse n'est pas vrai : plusieurs paragraphes ont souvent été numérotés pour un même fragment.

107. Ces éléments sont une mise en relation très ponctuelle de la Copie avec une édition de PortRoyal (mais ciblée car elle porte sur la comparaison entre les religions juive, chrétienne et musulmane) : 132 correspond à un $\S$ retenu p. 136 de l'édition de $1678 ; 138$ correspond à un $\S$ retenu p. 142-143 de l'édition de $1678 ; 127$ correspond à un $\S$ retenu p. 132 de l'édition de 1678 ; 83 correspond à un $\S$ retenu p. 87 de l'édition de 1678 . On remarquera que le décalage de 4-5 pages est constant. L'écriture des chiffres est différente de la concordance avec le RO. La main qui a écrit ces quatre éléments de concordance a aussi ajouté le numéro de la page 122.

108. Par Louis Périer? L'écriture le supposerait selon Z. Tourneur (Ibid., 1842, p. 13). Tourneur dit que les autres numéros sont plus tardifs.

109. L'écriture est différente des références ajoutées à la sanguine p. 259 vº et suivantes (voir cette étude). 
110. Quinze jours avant celui de Jean Guerrier.

111. La pagination de $C_{1}$ a dû être faite avant la phase de repérage des textes par Étienne pour l'édition de 1678. Sinon comment expliquer les marques ajoutées à la sanguine p. $259 \mathrm{v}^{\circ}$ et suivantes? (voir l'étude ci-dessus). Louis Périer avait 27 ans en 1678.

112. À moins que ce soit l'écriture de Marguerite.

113. Ce numéro de page a peut-être été ajouté à cette occasion.

114. Références Sellier : 41, 48, 78, 88, 92, 111, 115 et 116, 127, 135, 149, 165, 186, 207 et 210, 228, 230, 230, 230, 230 (2), 276, 276.

115. Que signifient J et $\mathrm{N}$ ? Faut-il y voir les initiales de collaborateurs de Bossut (J[ean le Rond d'Alembert] et $\mathrm{N}$ [icolas Condorcet]) ?

116. Ibid.p. 30.

117. La pagination tient compte des pages blanches.

118. Un des paragraphes concernés apparaît dans les exemplaires imprimés en 1669 mais a été supprimé avant l'édition de 1670.

119. L'encre semble être la même que le B cerclé ajouté en marge.

INDEX

Mots-clés : Pascal, Pensées, copies

\section{AUTEUR}

GILLES PROUST

CERHAC. Université Blaise Pascal 